\title{
Field and Shape Reconstruction in Fluid Dynamics
}

\author{
Dissertation \\ zur Erlangung des \\ mathematisch-naturwissenschaftlichen Doktorgrades \\ "Doctor rerum naturalium" \\ der Georg-August-Universität Göttingen
}

vorgelegt von

Qazi Muhammad Zaigham Zia

aus Bhoner Kaswal, Rawalpindi (Pakistan)

Göttingen 2011 
Referent: $\ldots \ldots \ldots \ldots \ldots \ldots \ldots \ldots \ldots \ldots \ldots \ldots \ldots \ldots$ Prof. Dr. Roland Potthast

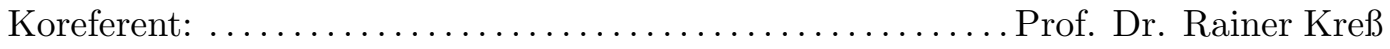
Tag der mündlichen Prüfung: .............................. 03.05.2011 
To

Ammi Jee and Uzma Zia 



\section{Acknowledgments}

The completion of this thesis owes much support, help and understanding of many people. I would like to take this opportunity to express my deepest appreciation to all of them.

First of all, I would like to thank my supervisor Prof. Dr. Roland Potthast for his support, guidance and critical evaluation of my work. I am also thankful to him for being understanding, encouraging, and most of all, patient during my journey of research.

I am highly grateful to Prof. Dr. Rainer Kress for taking time to help me despite his own very packed schedule. His valuable feedback and kind involvement led to significant improvements in this work. I would like to extend my appreciation to Prof. Dr. Gert Lube, especially for his amazingly prompt response each time I sought his advice or help.

Many thanks to all of my colleagues at the institute especially Boris, Robert and Ahmet for their moral support. A special thank goes to Dr. Jochen Schulz and to Dr. Olha Ivanyshyn for sparing time to help me in proof-reading part of the dissertation.

I am certainly indebted to the Higher Education Commission of Pakistan (HEC) for their financial support without which it would be impossible for me to pursue doctoral studies in Germany.

My acknowledgment will remains incomplete if I do not mention companionship of my friends in Göttingen and in Reading especially Haroon bhai, Tayyab, Rukhsar, Raja Shahid and Rehana for their emotional backing, humor, honesty and the wonderful time we shared in the last couple of years.

I am fully appreciative and thankful to my wife Mubeen and my little princess Khadija Zia for their unconditional love, care and patience which kept me afloat when I was in deep waters.

Finally I could not have completed this dissertation without the support of my whole family in Pakistan. My mother and sister whom this dissertation is dedicated to, has been a constant source of love, concern, support and strength all these years. I have to give a special mention for the unlimited support and care given by Teejan (Q. M. Atiq-Ur-Rahman) and Babu Jee (Q. M. Tajammul Farooq) through out my life. A special thanks goes to anti Farkhanda, Nani Amma, Sardar uncle and Shahzad Bhai. Thank you all for your concern, trust and prayers for me which are my strength indeed. 



\begin{abstract}
Inverse problems are concerned with the reconstruction of quantities from remote measurements. Inverse fluid flow problems are important for many applications, for example for determining the state of the atmosphere from measurements on the planets surface and further remote sensing techniques. Here, we investigate the reconstruction of some fluid flow and shape reconstruction for inclusions within the flow from boundary measurements. As a model problem we consider the Oseen equation, which is obtained by linearizing the Navier-Stokes equations.

In a first step we develop a point source method for the reconstruction of flow field from remote measurements. In contrast to field reconstructions in acoustics or electromagnetics, here we need a proper setup of the scheme as the fundamental solution of the Oseen equation is not symmetric or anti-symmetric in its arguments; moreover the null-spaces of the integral operators under consideration are no longer trivial, such that the corresponding convergence analysis of the point source method is particularly difficult.

Further we extend our study to develop methods to test for analytic extensibility in fluid dynamics for the inverse fluid flow problems. We study and analyze three different approaches for the analytical continuation, the range test, the no-response test and a convergence test. We prove the convergence of these methods when applied to the Oseen equation. In particular, we exhibit a new approach to show convergence of the no-response test. A strong relationship between the convergence test and the no response test is shown.

A numerical demonstration of the point source method and the convergence test is presented to exhibit the feasibility of these methods. To carry out the reconstructions we employ either domain sampling or the LASSO scheme is used for the reconstruction of flow field and the shape of unknown obstacles.
\end{abstract}





\section{Zusammenfassung}

Inverse Probleme beschäftigen sich mit der Rekonstruktion von Größen aus Fernerkundungsdaten. Inverse Strömungsmechanik ist wichtig für viele Anwendungen, zum Beispiel um den Zustand der Atmosphäre aus Messungen an der Oberfläche des Planeten und mit Hilfe weiterer Fernerkundungstechniken zu bestimmen. Hier untersuchen wir die Rekonstruktion einer Strömung und die Gestaltsrekonstruktion von Inklusionen in dieser Strömung aus Randwertmessungen. Als Modellproblem betrachten wir die Oseen Gleichung, die durch eine Linearisierung der Navier-Stokes Gleichungen entsteht.

In einem ersten Schritt entwickeln wir eine Punktquellenmethode für die Rekonstruktion der Strömung aus Fernerkundungsdaten. Im Unterschied zu Feldrekonstruktionen in der Akustik oder Elektromagnetik brauchen wir hier einen etwas anderen Ansatz, da die Grundlösung der Oseen Gleichung nicht symmetrisch oder Antisymmetrisch in ihren Argumenten ist; Ferner sind die Nullräume der Operatoren nicht-trivial, so dass die zugehörige Konvergenzanalysis der Punktquellenmethode einige Schwierigkeiten bietet.

Weiter erweitern wir unsere Untersuchungen auf Tests zur analytischen Fortsetzbarkeit in der Strömungsmechanik für inverse Flußprobleme. Wir studieren und analysieren drei verschiedene Methoden für die analytische Fortsetzung, den Range Test, den No Response Test und einen Konvergenztest. Wir zeigen die Konvergenz dieser Methoden in ihrer Anwendung auf die Oseen Gleichung. Insbesondere entwickeln wir eine neue Beweistechnik um die Konvergenz des No Response Tests zu beweisen. Es wird ferner eine starke Verwandschaft zwischen dem Konvergenztest und dem No Response Test nachgewisen.

Eine numerische Demonstration der Punktquellenmethode und des Konvergenz Tests wird präsentiert, um die Eigenschaften dieser Methoden zu zeigen. Um die Rekonstruktionen durchzuführen haben wir entweder "Domain Sampling" oder das LASSO Schema genutzt zur Ermittlung der Strömungsfelder und der Gestalt unbekannter Einschlüsse. 



\section{Contents}

1 Introduction $\quad 1$

$\begin{array}{lll}\text { I Theory } & 7\end{array}$

2 Basic Tools 9

2.1 Sobolev Space . . . . . . . . . . . . . . . . . . . 9 9

2.2 Fundamental Solution . . . . . . . . . . . . . . . . . . 13

2.3 Integral Operators . . . . . . . . . . . . . . . . . . . . . . . 15

2.4 Tikhonov Regularization . . . . . . . . . . . . . . . . . . . . 17

2.5 Nyström's Method . . . . . . . . . . . . . . . . . . 21

3 Direct Problem of Oseen Flow $\mathbf{2 5}$

3.1 Oseen Equation . . . . . . . . . . . . . . . . . . . 25

3.1.1 Derivation of the Fundamental Solution . . . . . . . . . 27

3.2 Direct Problem . . . . . . . . . . . . . . . . . . . 32

4 Field Reconstructions by the Point Source Method 51

4.1 Approximation with a Single-Layer Potential . . . . . . . . . . . 52

4.2 Uniqueness of Inverse problem . . . . . . . . . . . . . . . . 60

4.3 The Point Source Method for Oseen Flow . . . . . . . . . . . . . 61

5 Tests for Analytical Continuation $\quad \mathbf{6 5}$

5.1 Range Test . . . . . . . . . . . . . . . . . 66

5.2 No Response Test . . . . . . . . . . . . . . . . . . . . 69

5.3 Convergence Test . . . . . . . . . . . . . . . . 74

$\begin{array}{ll}\text { II Numerics } & 79\end{array}$

6 Domain Sampling $\quad 81$

6.1 Direct Problem . . . . . . . . . . . . . . . . . . . 81 
6.2 Point Source Method . . . . . . . . . . . . . . . . . . . . 84

6.3 Convergence Test . . . . . . . . . . . . . . . . . 97

7 LASSO Scheme $\quad 101$

7.1 LASSO Algorithm . . . . . . . . . . . . . . . . . . . . 101

7.1.1 Smoothness Criteria . . . . . . . . . . . . . 103

7.1 .2 Domain Splitting . . . . . . . . . . . . . . 105

7.2 Numerical Examples . . . . . . . . . . . . . . . . . . . . . . 108

$\begin{array}{ll}\text { III Appendix } & 115\end{array}$

$\begin{array}{lr}\text { A Background from Fluid Dynamics } & 117\end{array}$

A.1 Equation of Continuity . . . . . . . . . . . . . . . . . . 118

A.2 Equation of Motion . . . . . . . . . . . . . . . . 119

$\begin{array}{lr}\text { Bibliography } & 125\end{array}$ 


\section{Chapter 1}

\section{Introduction}

Inverse problems are those problems where we want to find the cause for a desired or an observed effect. They are mainly given by applications and have been studied for nearly a century. Most of the inverse problems are "ill-posed" in nature. Ill-posed means that they do not fulfill Hadamard's classical requirements [18], i.e., solutions of an inverse problem might not exist for all data, might not be unique, or might be unstable with respect to data perturbations. Usually the direct problems are simpler or more well studied than the inverse problems. These kind of problems appear in a large variety of applied sciences, such as medical diagnostics, computerized tomography, electrical impedance tomography, geophysics, and ocean acoustic tomography.

Nevertheless, the inverse fluid problems which are of fundamental importance for many applied areas ranging from industrial process monitoring to numerical weather prediction are still a relatively new area of research. In this monograph we study the inverse problem in fluid dynamics. More precisely we will search for the unknown inclusions and the velocity field in viscous incompressible fluids.

Mathematically, the motion of the fluids are governed by the Navier-Stokes equations which are non-linear in nature [5,8]. In 1845 Stokes [43] suggested to linearize these equation by assuming the ratio of inertial to viscous forces is vanishingly small so that the convective term can be neglected. Few years later, in 1851 , Stokes presented an explicit solution for such flows in exterior domains [44]. However, the explicit solutions of Stokes equations did not provide any information about the wake region behind the object and are therefore unacceptable from a physical viewpoint. C. W. Oseen found another linear approximation of Navier-Stokes equation in 1910 [32]. The result of such an approximation is more successful compared to Stokes since Oseen described a paraboloidal wake region behind the obstacle.

In this monograph, as a model problem, for a bounded domain $D \subset \mathbb{R}^{2}$, we investigate the following two dimensional inverse exterior Dirichlet problem for the stationary Oseen equation 


$$
\mu \triangle u-\partial_{1} u-\nabla p=0, \quad \nabla \cdot u=0,
$$

subject to the boundary condition, for a vector field $f$,

$$
u=f \quad \text { on } \quad \partial D
$$

and the condition at infinity

$$
\lim _{x \rightarrow \infty} u(x)=u_{\infty}
$$

in the sense of uniform convergence with respect to all directions, whereas $u_{\infty}$ is a constant vector. Here the vector field $u=\left(u_{1}, u_{2}\right)^{T}$ and the scalar function $p$ are the velocity and pressure field respectively, also $\mu>0$ represents the kinematic viscosity of the fluid.

The goal here is to infer knowledge about a fluid or inclusions from measurements in particular areas or on the surface of such a fluid. More precisely, our inverse problem is to reconstruct the velocity field and determine the boundary of the obstacle $D$ from the knowledge of the fluid velocity on an open arc $\Lambda \in \mathbb{R}^{2} \backslash \bar{D}$. This inverse problem is ill-posed, due to the analyticity the construction of the solution $u$ to the Oseen problem in the exterior of $D$ with the measured velocity field on $\Lambda$. This inverse problem is non-linear since the solution depends non-linearly on the boundary curve $\partial D$.

Kress and Meyer (2000) [23] studied the inverse fluid flow problems and successively obtained the shape of an unknown obstacle with the help of a regularized Newton iteration method. This opened a new area of research in the field of inverse problems and gave a rise to an important question whether the other approaches which are developed for solving inverse problems in the scattering theory are applicable for inverse fluid flow problems.

Approaches which are used to solve the inverse problems in scattering theory can be divided into the following three groups:

\section{Iterative techniques}

In these techniques we use the model of full forward problems to solve the inverse problems. Since these techniques require the full knowledge of the direct problem, they produce very nice reconstruction results. However these techniques are computationally involved because of the use of many direct problems. Newton method, Landweber scheme, conjugate gradient method and the various versions of least square fits are examples of iterative schemes.

\section{Decomposition techniques}

The methods which belong to this group decompose the problem into two parts, i.e., the ill-posed part to reconstruct the field and the well-posed part to find the unknown obstacle. As compared to iterative methods these techniques 
do not need to solve the direct problem for reconstructions but they do need the boundary condition of the unknown objects. The well known examples are Kirsch-Kress method, Dual space method or point source method.

\section{Probe and sampling techniques}

In Probe and sampling techniques we construct an indicator function which is responsible to provide information about the location, shape and properties of the unknown obstacle. The main advantage of the Probe and sampling techniques is that they work even if the physical properties of the unknown object are unknown, or in other words these techniques do not need the boundary condition. No-response test, factorization method, probe method are the few examples of the probe and sampling techniques.

In this thesis we extend the point source method, the no-response test and the range test to the inverse Oseen problem. We also develop the convergence test to locate and reconstruct the shape of an unknown obstacle and to show the relations between the methods mentioned above. In order to justify these methods theoretically, we prove existence of the solution to the interior Dirichlet problem for the Oseen equations and provide some further results on the exterior Oseen problem. Moreover, denseness of the potential operators for the Oseen equation is shown. We provide a suitable formulation of the point source method and prove convergence of flow reconstructions. For the realization of the reconstruction when the inclusions are not known we employ domain sampling. We demonstrate the feasibility of the method for reconstructing one or several objects by numerical examples. The reconstruction of the shape of unknown obstacles for the inverse fluid flow problem is found via Line Adaptation for Singular Sources Object identification (LASSO) scheme [40].

In the next few paragraphs we present basic numerical algorithms which are investigated in this thesis.

The point source method was introduced by Potthast in 1996 [34] for the inverse obstacle scattering problem. Later on he and his collaborators $[6,11,25,26,28,35,36$, 38 ] successfully employed this technique to different problems in scattering theory. The method decomposes the ill-posed non-linear problem into an ill-posed linear problem and well-posed non-linear problem. In the first ill-posed step in inverse fluid flow problems, the point source method reconstructs the flow field via a back projection formula and with this knowledge the point source method solves the nonlinear but well posed problem to find zeros of the total field in a second step.

In 2003 Potthast, Sylvester and Kusiok [41] introduced the range test which solves the inverse problem in scattering theory to locate and to reconstruct the shape of an unknown obstacle while using only the knowledge of one incident wave and the measurement of the scattered field on some fixed curve around the observation data. In fluid dynamics we can apply the range test since the velocity field 
is coming from one side (we take it as one incident wave). The basic idea of the range test is to determine the maximal set onto which the velocity field may be analytically extended. The compliment of this analytically extended set is a subset of our unknown object since in the range test we do not use the boundary condition and that's why we have only an approximation of the unknown object.

The no response test that was introduced by Luke and Potthast in 2003 [27] and examines whether the unknown scatterer lies inside some test domains or not with the help of analytical continuation of the velocity field. The idea of the no response test is to construct an indicator function which is the supremum of reconstructed velocity field, called responses, on a test domain $G$, known as the maximal response $\mathfrak{T}$. With the help of this indicator function we probe the area and detect the region where we have small responses. The region which consists of small responses is the approximation of our reconstructed unknown obstacle.

At the end we develop the convergence test to locate and reconstruct the shape of an unknown obstacle, also it shows how these above methods are related to each other. We can consider this method as one from the group of probe and sampling techniques. We define an indicator function with the help of the Cauchy criterion on the two reconstructed velocity fields with different regularization parameters. This indicator function tells us where the reconstructed velocity field converges. This indicator function indicates the area which is a subset of our unknown obstacle. In this thesis we carry out the convergence test to observe the convergence of the point source method. The convergence test can be related to the no response test as well. Although the idea behind these two methods are entirely different however we can consider the convergence test as a variant of the no response test. In the no response test we calculate the response for one particularly chosen density such that the velocity field can be analytically extended to the exterior of the test domain $G$. For the convergence test this particular density is chosen to be the difference of two densities for different regularization parameters. For the analytical continuation of the reconstructed velocity fields corresponding to these densities, the Cauchy criterion is employed.

The idea of Domain Sampling is that we first construct an approximation domain $G$ and then test the convergence of the reconstruction on the boundary $\partial G$ or outside $G$. We carry out this procedure for many test domains to probe the area where the reconstruction is convergent. Naturally this leads us to a situation where we have many reconstructions of the velocity field. We combine these reconstructions by some masking operation and taking pointwise weighted averages.

In LASSO scheme we construct a circular curve which is sufficiently large to contain the unknown obstacles. With the help of an indicator function, which is found via the point source method, we implement the iteration procedure on the approximation domain so that the boundary of our test domain stretches towards those areas where our velocity field is small compared to the whole field. We repeat this procedure for $n \in \mathbb{N}$ iterations while for each iteration we create a new closed 
curve using the concept of parallel surfaces. For every iteration our newly obtained closed curve looses smoothness. To overcome this difficulty we introduce a smoothness criteria, our unknown obstacles belong to those areas where the velocity field is small. Thus after a number of iterations we reconstruct the boundaries of the unknown obstacles.

The thesis is divided into three parts. In the first part we focus on theoretical results for both the direct and the inverse problem. While in the second part we present a numerical study of the problem. We demonstrate the accuracy and efficiency of the inverse algorithms with the help of examples. The third part of the thesis is an appendix which consists of the basic theory of fluid dynamics.

Chapter 2 is dedicated to the basic tools which will be used later during the study of direct and inverse problems. We start with the introduction of Sobolev spaces and talk about the fundamental solution and its importance in the study of linear partial differential equations. Since for the solution of direct and inverse problems the boundary integral equation approach will be used we give a brief introduction of integral operators and some of their properties. We also discuss the importance of the Riesz theory for the solution of integral equations of the second kind. In Section 2.4 we discuss basic ideas of the ill-posed problems and their approximate solution via Tikhonov regularization. In the final Section 2.5 of this chapter we recall the Nyström's Method for the numerical solution of integral equations of the second kind.

Since solving inverse problems requires a solid knowledge of the corresponding direct problems, we include a detailed study on the direct problem of the Oseen equations in Chapter 3. In Section 3.1, we first present the weak formulation of the Oseen equations and then we construct the fundamental solution of the Oseen equations. We follow the standard tools for the formulation of the fundamental solution of the Oseen equations [16,33]. In Section 3.2 we use the boundary integral equation approach to prove the existence of the solution to the exterior and interior Dirichlet Oseen problem. In principle, for the existence of the solution of the exterior Dirichlet problem we follow Kress and Meyer [23]. For the interior Dirichlet problem for the Oseen equations, which had not been investigated in the literature so far, we present a new existence proof. We also give the proof of the interior Dirichlet problem using the classic Lax-Milgram theorem with restriction on viscosity parameter. To prove the denseness of the potential operators of the Oseen equations we introduce the adjoint Oseen equations and the corresponding potential theory which plays an important role in the inverse problem as well.

In Section 4.1, we formulate the basic tools for the point source method. In a first step we introduce the potential operators for the Oseen equations and the adjoint Oseen equations in a special setting of the $L^{2}$-spaces. With the help of these potential operators we are able to get an approximation of the point source $E(x-$.) on the boundary of the approximation domain $G_{x}$. In Section 4.3 we formulate the back projection formula for the reconstruction of the velocity field $u_{r e c}$. At the end 
we present the convergence of the reconstructed velocity field to the true velocity field.

In Section 5.1 we discuss the extension of the range test to the inverse fluid flow problem. We present a proof for the analytical continuation of the velocity field to the boundary of the test domain. In Section 5.2 we exhibit the applicability of the no response test to inverse fluid dynamics. We introduce a maximal response for a test domain $G$ with the help of which we prove that the velocity field is analytically extendable up to the exterior of the test domain for a small maximal response. We also prove a result which connects the no response test with the range test. In the last Section 5.3 we present the convergence test for the analytical continuation of the velocity field up to the exterior of a test domain $G$. Moreover, we show that the convergence test can be considered as a special case of the no response test. The convergence test can be used to prove the convergence of the point source method.

In the second part of this thesis we present a numerical study for the reconstruction of the field and the shape of an unknown obstacle via Domain Sampling and the $L A S S O$ schemes.

In Chapter 6 we present the domain sampling scheme. In Section 6.2, we demonstrate the point source method with the help of domain sampling technique. In a first step we describe in detail how to choose the approximation domain $G$ and then how to combine all the reconstructions for different test domains on a source point $x$ with the help of a masking procedure.

In the last Chapter 7 of this thesis we apply the LASSO scheme using the reconstructed velocity field from the point source method. We explain the concept of parallel surfaces and the smoothness criteria for the LASSO scheme in details. Also at the end we present numerical examples for a better understanding of the LASSO scheme.

The appendix gives a short introduction and a brief summary of the fluid dynamics. We derive the equation of continuity and the equation of motion for two dimensional incompressible flow. We discuss the viscosity and wake region in detail. We present the derivation of the Oseen equation from the Navier-Stokes equations.

To summarize, we analyzed the applicability of the point source method, the no response test, the range test and the convergence test to the inverse fluid flow problems by considering the Oseen equation. It will be highly interesting to observe these methods in a more complicated fluid dynamic problems, for example, the time dependent Oseen flow. The study of potential theory for the time dependent Oseen equation is more challenging. 


\section{Part I}

\section{Theory}





\section{Chapter 2}

\section{Basic Tools}

In this chapter we introduce some basic concepts from functional analysis and fluid dynamics which we apply in this monograph. We start this chapter by presenting a brief introduction to Sobolev spaces. In the second section we establish the well known Navier Stokes equation and discuss some useful properties of the fluid flow phenomena. As we will use the integral equation approach through out our study, the remaining part of the chapter introduces the necessary tools which are required to study the integral equation approach, both theoretically and numerically. We also discuss the basic concept of ill-posed and well-posed integral equations in detail.

\subsection{Sobolev Space}

It is observed that classical solutions of the problems which arise in the field of applied mathematics are not always sufficient enough. Some times, we have to introduce weak solutions to get good results. For this we need for example Sobolev spaces to deal with the weak derivatives. These spaces were introduced by the russian mathematician Sergei Sobolev. Here we will discuss the basic concept of Sobolev spaces which are used in this work. For a more detailed study of Sobolev spaces we refer the readers to [2], [7].

Before turning to Sobolev spaces we first introduce some function spaces, which are necessary for defining weak derivatives, such as the Lebesgue spaces ( $L^{P}$-spaces) and the spaces of continuously differentiable functions. In this section, unless otherwise stated, the Greek letter $\Omega$ shall always stand for an open bounded domain in $\mathbb{R}^{n}, n \in \mathbb{N}$.

Definition 2.1. For a non-negative integer $k$, the space of $k$-times continuously differentiable functions $C^{k}(\Omega)$ contains all real continuous functions $u$ defined in $\Omega$ together with all their derivatives $D^{\alpha} u$ of order $|\alpha| \leq k$, which are continuous in $\Omega$. For $k=0$ we have the space of continuous functions $C^{0}(\Omega)=C(\Omega)$. Also the space 
$C^{\infty}(\Omega)$ is defined as

$$
C^{\infty}(\Omega):=\bigcap_{k=0}^{\infty} C^{k}(\Omega) .
$$

Moreover, by $C_{0}^{k}(\Omega), 0 \leq k \leq \infty$ we denote the space of $k$-times continuously differentiable functions with compact support in $\Omega$.

Definition 2.2. The space $L^{p}(\Omega), 0 \leq p<\infty$ is the set of all Lebesgue measurable functions $u(x) \in \Omega$ such that the norm

$$
\|u(x)\|_{p, \Omega}:=\left(\int_{\Omega}|u(x)|^{p}\right)^{1 / p}
$$

is finite. It is observed that $L^{p}(\Omega)$ is a Banach space. Moreover, for $p=2$, the space $L^{2}(\Omega)$ becomes a Hilbert space under the scalar product

$$
\langle u, v\rangle_{L^{p}(\Omega)}=\int_{\Omega} u(x) v(x) d x .
$$

Definition 2.3. A function $\varpi: \Omega \rightarrow R$ is called a test function if it belongs to the space of infinitely differentiable functions $C_{0}^{\infty}(\Omega)$ with compact support.

We are now in the position to define the weak derivatives. We first assume that $u(x) \in C^{1}(\Omega)$ and $\varpi \in C^{\infty}$ is a test function. Then from integration by parts, we obtain,

$$
\int_{\Omega} u \varpi_{x_{i}} d x=-\int_{\Omega} u_{x_{i}} \varpi d x
$$

where the subscripts represents the derivative. The boundary terms vanishes because of the compact support of the test function in $\varpi \in \Omega$. If we generalize this idea and assume that $k$ is a non-negative integer and $\alpha$ is a $n$-tuple of non-negative integers $\alpha_{k}$ such that

$$
|\alpha|=\sum_{k=1}^{n} \alpha_{i}
$$

Then the $|\alpha|^{t h}$ order partial derivative of a function $u: \Omega \subset \mathbb{R}^{n} \rightarrow \mathbb{R}$ is given by

$$
\partial^{\alpha} u(x)=\frac{D^{\alpha} u(x)}{\partial x_{1}^{\alpha_{1}}, \cdots, \partial x_{n}^{\alpha_{n}}},
$$

and finally from integration by parts, we obtain,

$$
\int_{\Omega} u D^{\alpha} \varpi d x=(-)^{|\alpha|} \int_{\Omega} D^{\alpha} u \varpi d x .
$$


Definition 2.4. Let $u$ and $v$ are functions in the Lebesgue space $L^{1}(\Omega)$. We say that $v$ is the $\alpha^{\text {th }}$ weak derivative of $u$, if it satisfies the following equation

$$
\int_{\Omega} u \partial^{\alpha} \varpi=(-1)^{|\alpha|} \int_{\Omega} v \varpi
$$

for all test function $\varpi \in C_{0}^{\infty}(\Omega)$.

If the function is sufficiently smooth then the classical derivative is also a weak derivative, but the converse is not true. The main advantage to introduce the weak derivative is that we do not need the existence of the derivatives of smaller order like in the classical definition.

Lemma 2.5. A weak $\alpha^{\text {th }}$-partial derivative of $u$, if it exists, is uniquely defined.

Proof. See [12].

Definition 2.6. The Soboloev space $W^{m, p}(\Omega), 0 \leq 0<1$, of order $m$ is defined by

$$
W^{m, p}(\Omega):=\left\{u \in L^{p}(\Omega): D^{\alpha} u \in L^{p}(\Omega), 0 \leq|\alpha| \leq m\right\} .
$$

Here the derivatives $D^{\alpha} u$ are taken in the weak sense.

In the space $W^{m, p}(\Omega)$ we introduce the following norm

$$
\|u\|_{m, p}=\left(\sum_{0 \leq|\alpha| \leq m}\left\|D^{\alpha} u\right\|_{L^{p}(\Omega)}^{p}\right)^{1 / p} \quad \text { if } \quad 0 \leq|\alpha|<\infty .
$$

For the special case $p=2$ we denote the Sobolev space by $W^{m}$ and it becomes a Hilbert space under the scalar product defined in Definition 2.2.

Definition 2.7. Let $\partial \Omega$ be the boundary of a simply connected domain $\Omega \subset \mathbb{R}^{2}$ of class $C^{k}, k \in \mathbb{N}$. If $\partial \Omega$ is a regular and $2 \pi$-periodic parametric representation such that $\partial \Omega=\{\vartheta(t): t \in[0,2 \pi)\}$, then the sobolev space on the boundary, $H^{p}(\partial \Omega)$ for $0 \leq p \leq k$, is the space of all functions $\phi \in L^{2}(\partial \Omega)$ with the property $\phi \circ \vartheta \in H^{p}[0,2 \pi]$. Here $\phi \circ \vartheta$ denotes the $2 \pi$-periodic function given by $(\phi \circ \vartheta)(t)=\phi(\vartheta(t)), t \in \mathbb{R}$. The scalar product and norm on $H^{p}(\partial \Omega)$ are defined through the scalar product on $H^{p}[0,2 \pi]$ by

$$
(\phi, \psi)_{H^{p}(\partial \Omega)}:=(\phi \circ \vartheta, \psi \circ \vartheta)_{H^{p}[0,2 \pi]} .
$$

Without loss of generality we have chosen a $[0,2 \pi)$ parametric domain. However we must allow the different parametric representation for the boundary $\partial \Omega$. Therefore we need to know that whether the above definition is valid for more than one parametric representation or not. The answer is yes and is explained in the following theorem. 
Theorem 2.8. Let $\partial \Omega$ satisfy the assumptions of Definition 2.7. Assume that $\vartheta$ and $\hat{\vartheta}$ are two different regular $2 \pi$-periodic parametric representations of $\partial \Omega$ such that $\partial \Omega=\{\vartheta(t): t \in[0,2 \pi)\}$ and $\partial \Omega=\{\hat{\vartheta}(t): t \in[0,2 \pi)\}$. Then the Sobolev spaces

$$
H^{p}(\partial \Omega):=\left\{\phi \in L^{2}(\partial \Omega): \phi \circ \vartheta \in H^{p}[0,2 \pi]\right.
$$

with the scalar product

$$
(\phi, \psi)_{H^{p}(\partial \Omega)}:=(\phi \circ \vartheta, \psi \circ \vartheta)_{H^{p}[0,2 \pi]}
$$

and

$$
\tilde{H}^{p}(\partial \Omega):=\left\{\phi \in L^{2}(\partial \Omega): \phi \circ \hat{\vartheta} \in H^{p}[0,2 \pi]\right.
$$

with the scalar product

$$
(\phi, \psi)_{\tilde{H}^{p}(\partial \Omega)}:=(\phi \circ \hat{\vartheta}, \psi \circ \hat{\vartheta})_{H^{p}[0,2 \pi]}
$$

are homeomorphic.

Proof. See Theorem 8.14 in [22].

We exhibit the connection between Sobolev spaces on a domain $\Omega$ and Sobolev spaces on its boundary $\partial \Omega$ by the following trace theorem. Before this we need to introduce the concept of trace. For functions defined on the closure $\bar{\Omega}$ with clearly defined values on the boundary $\partial \Omega$ and the reasonable restriction of the function to the boundary $\partial \Omega$ is called trace. Furthermore an operator

$$
T: C^{\infty}(\bar{\Omega}) \rightarrow C^{\infty}(\partial \Omega)
$$

is said to be a trace operator if it maps a function onto its trace such that

$$
T u:=\left.u\right|_{\partial \Omega} .
$$

Theorem 2.9. Assume that $\Omega$ is a $C^{k-1,1}$ domain. For $\frac{1}{2}<s \leq k$ the trace operator $T$ has a unique extension to a bounded linear operator

$$
T: W^{s}(\Omega) \rightarrow W^{s-\frac{1}{2}}(\partial \Omega)
$$

and this extension has a continuous right inverse. If $u \in C^{\infty}(\bar{\Omega})$ then

$$
\|u\|_{W^{s-\frac{1}{2}}} \leq C\|u\|_{W^{s}(\Omega)}
$$

Proof. See Theorem 3.37 in [29]. 


\section{$2.2 \quad$ Fundamental Solution}

Fundamental solutions play an important role in the theory of partial differential equation especially when we deal with existence and regularity of the solutions. If we have an explicit fundamental solution for a linear partial differential equation then with the help of convolution process we can assemble more complicated desired solutions. To understand the concept of fundamental solutions we first introduce these definitions.

Definition 2.10. A linear functional $f$ is called a distribution on a bounded domain $\Omega$ if for every compact set $A \subset \Omega$ their exists a constant $C$ and $n \in \mathbb{N}$ such that for all $\varphi \in C_{0}^{\infty}(A)$ the following inequality holds

$$
|f(\varphi)| \leq C \sum_{|\alpha|<n} \sup \left|\partial^{\alpha} \varphi\right|
$$

The set of all distributions on $\Omega$ is denoted by $\mathcal{D}^{\prime}(\Omega)$.

Definition 2.11. The distribution derivative of a function $u \in \mathcal{D}^{\prime}(\Omega)$ is defind by

$$
\left(\partial_{i} u\right)(\varphi)=-u\left(\partial_{i} \varphi\right), \quad \varphi \in C_{0}^{\infty}(\Omega) .
$$

It is trivial that higher orders of the derivative can be obtained by induction. From the above definition we can see that every distribution on a bounded domain $\Omega$ has a distribution derivative of any order. Thus we have the following definition of fundamental solution.

Definition 2.12. A distribution $E \in \mathcal{D}^{\prime}(\Omega)$ is called the fundamental solution of the linear partial differential operator $\mathfrak{L}$ if it satisfies

$$
\mathfrak{L} E=\delta .
$$

Here $\delta$ is the Dirac's $\delta$ distribution and for any test function $\varphi \in C_{0}^{\infty}(\Omega)$ it is defined as

$$
\delta(\varphi):=\varphi(0)
$$

Remark: Fundamental solutions are very important for obtaining the classical solution of an inhomogeous partial differential equation $\mathfrak{L} u=g$. Consider $u=E * g$, $\mathrm{E}$ is the fundamental solution of the operator $\mathfrak{L}$, then

$$
\begin{aligned}
\mathfrak{L} u & =\mathfrak{L}(E * g) \\
& =\mathfrak{L}(E) * g \\
& =\delta * g \\
& =g .
\end{aligned}
$$

Thus $E * g$ is a solution in the classical sense. 
Theorem 2.13 (Malgrange-Ehrenpreis). For every differential operator $\mathfrak{L}$ their exist a distribution $E \in \mathcal{D}^{\prime}$ such that $\mathfrak{L} E=\delta$.

Proof. For the proof we refer the reader to [14].

The Malgrange-Ehrenpreis theorem assures the existence of an fundamental solution for any differential operator. Since our main area of study is fluid dynamics we present as an example the derivation of the fundamental solution of the Stokes equation,

$$
\Delta u-\nabla \tilde{p}=0, \quad \nabla \cdot u=0, \quad \text { in } \Omega .
$$

Assume that $\Phi(z)$ is an arbitrary function on $\mathbb{R}$, which is smooth for $z \neq 0$ and $\delta_{i j}$ is the Kronecker delta. To obtain the fundamental solution, following [16], we consider the second order symmetric tensor field $E$ and vector field $e$ defined by the relation

$$
\begin{gathered}
E_{i j}(x-y)=\left(\delta_{i j} \Delta-\frac{\partial^{2}}{\partial y_{i} \partial y_{j}}\right) \Phi(|x-y|), \\
e_{j}(x-y)=-\frac{\partial}{\partial y_{j}} \Delta \Phi(|x-y|),
\end{gathered}
$$

for $i, j=1,2$ and $x, y \in \mathbb{R}^{2}$. Applying Laplace operator on equation (2.2.1), we have

$$
\Delta E_{i j}(x-y)=\left(\delta_{i j} \Delta^{2}-\frac{\partial^{2}}{\partial y_{i} \partial y_{j}} \Delta\right) \Phi(|x-y|) .
$$

Similarly taking the derivative $\partial / \partial y_{i}$ of equation $(2.2 .2)$

$$
\frac{\partial}{\partial y_{i}} e_{j}(x-y)=-\frac{\partial^{2}}{\partial y_{i} \partial y_{j}} \Delta \Phi(|x-y|) .
$$

Subtracting equation (2.2.4) from (2.2.3), we obtain

$$
\Delta E_{i j}(x-y)-\frac{\partial}{\partial y_{i}} e_{j}(x-y)=\delta_{i j} \Delta^{2} \Phi(|x-y|),
$$

also using the Einstein's convention we get

$$
\frac{\partial}{\partial y_{j}} E_{i j}(x-y)=0 .
$$

On the right hand side of equation (2.2.5) we have the biharmonic operator. The fundamental solution of biharmonic equation $\Delta^{2} \Phi(|x-y|)=0$ is given by

$$
\Phi(|x-y|)=|x-y|^{2} \ln (|x-y|) / 8 \pi .
$$


Substituting back the value of equation (2.2.5), we have

$$
\begin{aligned}
E_{i j}(x-y) & =-\frac{1}{4 \pi}\left(\delta_{i j} \ln \frac{1}{|x-y|}+\frac{\left(x_{i}-y_{i}\right)\left(x_{j}-y_{j}\right)}{\left|x-y^{2}\right|}\right), \\
e_{j}(x-y) & =\frac{1}{2 \pi} \frac{x_{j}-y_{j}}{|x-y|^{2}} .
\end{aligned}
$$

For $x-y=z \neq 0$ we can write the last two equations (2.2.8) and(2.2.9) as

$$
\begin{aligned}
& E(z)=\frac{1}{4 \pi}\left\{\ln |z| I+\frac{z z^{t}}{|z|^{2}}\right\}, \\
& e(z)=\frac{1}{2 \pi} \operatorname{grad}(\ln |x|) .
\end{aligned}
$$

The pair $E$ and $e$ is called the fundamental solution of the Stokes equation.

\section{$2.3 \quad$ Integral Operators}

An equation which contains an unknown function $\varphi$ and its definite integral is known as an integral equation. If the limits of the integral are constant then it is called a Fredholm integral equation otherwise it is known as a Voltera integral equation. Also if the unknown function $\varphi$ is strictly inside the integral then the equation is said to be of first kind, and if the unknown function $\varphi$ appears inside as well as outside the integral it is known as the equation of second kind, i.e., for a continuous function $K(x, y)$,

$$
\int_{a}^{b} K(x, y) \varphi(y) d y=f(x) \quad x \in[a, b]
$$

and

$$
\varphi(x)-\int_{a}^{b} K(x, y) \varphi(y) d y=f(x) \quad x \in[a, b]
$$

are the typical examples of Fredholm integral equations of first and second kind respectively.

Integral equations occur in different areas of applied mathematics and physics. These type of equations provide a powerful technique for solving a variety of practical problems. For several reasons integral equations are the preferred methods for analyzing differential equations of initial or boundary value problems. The straight forward benefit of using integral equations rather then differential equation is that it combines all the conditions specified in initial or boundary value problem into a single integral equation. Another advantage of integral equations are the reduction of the dimension of the problem, for example, a boundary value problem for a partial differential equation with two independent variables transforms into an integral equation which have a unknown function of only one variable. 
In operator notation, we can write the integral equation of first and second kind as

$$
A \varphi=f
$$

and

$$
\varphi-A \varphi=f .
$$

Definition 2.14. Let $\Omega \subset \mathbb{R}^{m}$ be a nonempty compact and Jordan measurable set that coincides with the closure of its interior. Then the operator $A: C(\Omega) \rightarrow C(\Omega)$, defined by

$$
(A \varphi)(x):=\int_{\Omega} K(x, y) \varphi(y) d y, \quad x \in \Omega,
$$

is called an integral operator with continuous kernel provided that $K: \Omega \times \Omega \rightarrow \mathbb{C}$ is a continuous function.

The integral operator defined in (2.3.5) is bounded (see Theorem 2.8 in [22]) under the norm

$$
\|A\|_{\infty}=\max _{x \in \Omega} \int_{\Omega}|K(x, y)| d y .
$$

The question of uniqueness and existence of the solution obtained via integral equations is equally important as in differential equations. The uniqueness and existence of the solution of the integral equation of second kind (2.3.4) can be established by the Neumann series provided that $\|A\|<1$ (see [22]). Neumann series has a major draw back that it is only applicable for the integral equation of the second kind with sufficiently small kernels. Therefore we need some other tools to establish the uniqueness and existence of integral equation. This leads us to the introduction of compact operators.

Definition 2.15. Let $X$ and $Y$ are two normed spaces. Then a linear operator $A: X \rightarrow Y$ is called compact if it maps each bounded set in $X$ into a relatively compact set in $Y$.

Theorem 2.16. The integral operator with continuous kernel is a compact operator on $C(\Omega)$.

Proof. See [22].

With the Theorem 2.16 we are now in position to say that the integral equations of first (2.3.3) and second kind (2.3.4) contains the compact operator $A: X \rightarrow X$. A compact operator $A$ on an infinite dimensional space $X$ cannot have a bounded inverse since $A^{-1} A=I$ is not a compact operator on any infinite dimensional space. This is a very important conclusion. It shows that the integral equation of first kind with continuous kernel is not boundedly invertible, this leads us to the phenomena of ill-posed problems. We discuss this phenomena in detail in next section. 
Now coming to the integral equation of second kind, we can write equation (2.3.4) as

$$
L \varphi=f
$$

where $L:=I-A$ with $I$ is the identity. We have developed tools for analyzing uniqueness and existence of the integral equation of second kind. Due to Frigyes Riesz $(\mathbf{1 8 8 0}-\mathbf{1 9 5 6 )}$ we know that the null space of the operator $L$, i.e.,

$$
N(L):=\{\varphi \in X: L \varphi=0\}
$$

is a finite dimensional subspace and its range is a closed linear subspace. The boundedness of the inverse operator $L$ is confirmed by the following fundamental result of the Riesz theory [22].

Theorem 2.17. Let $X$ be the normed space and $A: X \rightarrow X$ is a compact linear operator. Then the operator $I-A$ is injective if and only if it is surjective. Also the inverse operator $(I-A)^{-1}: X \rightarrow X$ is bounded provided that $I-A$ is injective.

With this knowledge we are able to conclude the following result which ensures the uniqueness and existence of the integral equation of second kind.

Corollary 2.18. Let $A: X \rightarrow X$ be a compact linear operator on a normed space $X$. If the homogeneous equation

$$
\varphi-A \varphi=0
$$

only has the trivial solution $\varphi=0$, then for each $f \in X$ the corresponding inhomogeneous equation (2.3.4) has a unique solution $\varphi \in X$ and this solution depends continuously on $f$.

Thus with the help of the Riesz theory we are able to solve integral equations of the second kind, which arise in many practical problems in the theory of fluid dynamics, electromagnetic or acoustics.

\subsection{Tikhonov Regularization}

In 1923, Hadamard [18] defined a well-posed problem by postulating the following three properties:

- Existence of the solution.

- Uniqueness of the solution.

- Continuous dependence of the solution on the data.

If one of the above properties is violated then we can say that the problem is illposed. We give the definition of a well-posed problem in the setting of an operator equation. 
Definition 2.19. Consider a bounded linear operator $A: X \rightarrow Y$ from a normed space $X$ to a normed space $Y$. If the operator $A$ is bijective and its inverse is continuous, then the equation

$$
A \varphi=f
$$

is called well-posed, otherwise it is called ill-posed.

Theorem 2.20. Let $X$ and $Y$ be two normed spaces and let $A: X \rightarrow Y$ be a compact linear operator. Then the integral equation of the first kind

$$
A \varphi=f
$$

is ill-posed provided that the normed space $X$ is infinite dimensional.

Proof. On contrary we assume that inverse operator $A^{-1}$ is bounded then the product of $A^{-1} A=I$ is compact on $X$ (see Theorem 2.16 of [22]), which is not possible because the identity operator $I$ is compact only on finite dimensional spaces (compare Theorem 2.20 in [22]).

This theorem tells us that the linear integral equations of the first kind with continuous or weakly singular kernels are examples of ill-posed problems. The third postulate described by Hadamard for well-posed problems is difficult for integral equation of the first kind. Due to the discontinuity of the inverse operator $A^{-1}$ small changes in the data leads to unstable solutions. In order to obtain a stable solution we have to consider the third condition postulated by Hadamard.

The basic idea to deal with the instability of such ill-posed problems is to find a bounded approximation $R_{\alpha}$ to the unbounded operator $A^{-1}$ depending on some parameter $\alpha$. The strategy to find such a bounded approximation $R_{\alpha}$ is known as the regularization scheme.

Definition 2.21. A family of bounded linear operators defined on the normed spaces $X$ and $Y$, such that

$$
R_{\alpha}: Y \rightarrow X, \quad \alpha>0
$$

is called a regularization scheme for an injective operator $A: X \rightarrow Y$, if

$$
\lim _{\alpha \rightarrow 0} R_{\alpha} A \varphi=\varphi, \quad \varphi \in X .
$$

The limit in equation (2.4.2) describes that $R_{\alpha}$ tends pointwise to $A^{-1}$. In the following theorem we observe two fundamental properties of the regularization scheme $R_{\alpha}$ for compact operators.

Theorem 2.22. Let $A: X \rightarrow Y$ be the compact operator on the normed spaces $X$ and $Y$ with $\operatorname{dim} X=\infty$ and a regularization scheme $R_{\alpha}, \alpha>0$. Then the family $R_{\alpha}, \alpha>0$ of bounded operators cannot be uniformaly bounded with respect to $\alpha$ and the operators $R_{\alpha}$ can not be norm convergent as $\alpha \rightarrow 0$. 
Proof. Following [22], we assume on contrary basis that the regularization operator $R_{\alpha}$ is bounded such that $\left\|R_{\alpha}\right\|<C$ for all $\alpha>0$ with some constant $C$. For all $f \in A(X)$ and in the view of equation (2.4.2) we have $R_{\alpha} f \rightarrow A^{-1} f$ when $\alpha \rightarrow 0$. Due to our assumption we can deduce that $\left\|A^{-1} f\right\| \leq C\|f\|$, i.e., $A^{-1}: A(X) \rightarrow X$ is bounded. Theorem 2.20 leads us to a contradiction.

We prove the second statement with the assumption that we have the norm convergence. Then there exists $\alpha>0$ such that $\left\|R_{\alpha} A-I\right\|<1 / 2$. Now for all $f \in A(X)$ we have

$$
\begin{aligned}
\left\|A^{-1} f\right\| & =\left\|A^{-1} f-R_{\alpha} f+R_{\alpha} f\right\| \leq\left\|A^{-1} f-R_{\alpha} A A^{-1} f\right\|+\left\|R_{\alpha} f\right\| \\
& \leq\left\|A^{-1} f\right\|\left\|I-R_{\alpha} A\right\|+\left\|R_{\alpha} f\right\| \leq \frac{1}{2}\left\|A^{-1} f\right\|+\left\|R_{\alpha}\right\|\|f\| .
\end{aligned}
$$

This implies $\left\|A^{-1} f\right\| \leq 2\left\|R_{\alpha}\right\|$ or in other words $A^{-1}: A(X) \rightarrow X$ is bounded. This leads us to the same contradiction as above.

The regularization scheme converges pointwise such that $R_{\alpha} f \rightarrow A^{-1}$ for $\alpha \rightarrow 0$ holds for all $f \in A(X)$. On the other hand if the data is perturbed by some noise such that $\left\|f^{\delta}-f\right\| \leq \delta$, then for a regularization parameter $\alpha$, we find an approximate solution $\varphi^{\delta}$ such that

$$
\varphi_{\alpha}^{\delta}:=R_{\alpha} f^{\delta}
$$

To estimate the error in the solution we write,

$$
\begin{aligned}
\varphi_{\alpha}^{\delta}-\varphi & =R_{\alpha} f^{\delta}-\varphi \\
& =R_{\alpha} f^{\delta}-R_{\alpha} f+R_{\alpha} f-\varphi \\
& =R_{\alpha} f^{\delta}-R_{\alpha} f+R_{\alpha} A \varphi-\varphi .
\end{aligned}
$$

Using the triangle inequality we obtain

$$
\begin{aligned}
\left\|\varphi_{\alpha}^{\delta}-\varphi\right\| & \leq\left\|R_{\alpha} f^{\delta}-R_{\alpha} f\right\|+\left\|R_{\alpha} A \varphi-\varphi\right\| \\
& \leq \delta\left\|R_{\alpha}\right\|+\left\|R_{\alpha} A \varphi-\varphi\right\| .
\end{aligned}
$$

Thus we decomposed the error into two parts, the first term reflects the data error and the second term expresses the error between the regularization operator $R_{\alpha}$ and the inverse operator $A^{-1}$. Theorem 2.22 tells us that the first term is not uniformly bounded with respect to $\alpha$. It means this term increases as $\alpha \rightarrow 0$, due to the unboundedness of the regularization operator $R_{\alpha}$. The second term decreases as $\alpha \rightarrow 0$ because of the limit defined in equation (2.4.2). This leads us to a difficult task how to choose the regularization parameter $\alpha$ such that we have an acceptable error level for the regularized solution. The accuracy of the approximation requires small error $\left\|R_{\alpha} A \varphi-\varphi\right\|$, i.e., a small parameter $\alpha$ and at the same time for the stability of the problem we need a large $\alpha$. Thus we have some kind of compromise between the accuracy and the stability for the choice of $\alpha$. The choice of the regularization parameter depending on the error level $\delta$ is called a strategy. 
Definition 2.23. A strategy is called regular if for all $f \in A(X)$ and all $f^{\delta} \in Y$ with $\left\|f^{\delta}-f\right\| \leq \delta$ we have

$$
R_{\alpha(\delta)} f^{\delta} \rightarrow A^{-1} f, \quad \delta \rightarrow 0 .
$$

In the area of inverse problems there are several strategies for the choice of regularization parameter $\alpha$, for a comprehensive view see for example [9]. We can divide them into the class of a priori and a posteriori strategies. The a priori strategies would be based on some additional information about the problem, for example the information about the smoothness properties of the exact solution. These strategies are not widely used because this kind of information is usually not available. So we mainly focused on the a posteriori strategies of which one is the following discrepancy or residual principle introduced by Morozov [31].

Definition 2.24 (Discrepancy Principle). The regularization parameter $\alpha$, for the error level $\delta$, should be chosen such that

$$
\left\|A R_{\alpha} f^{\delta}-f^{\delta}\right\|=\gamma \delta
$$

with some fixed parameter $\gamma \geq 1$.

The basic idea of the Tikhonov regularization is to approximate the fundamental solution by an element in the range of some integral operator $A$ between two Hilbert spaces $X$ and $Y$. So in the Tikhonov regularization we are interested to minimize the residual $\|A \varphi-f\|$ for all $f \in X$. For the stability of the minimization procedure a penalty term $\alpha\|\varphi\|$, with a regularization parameter $\alpha>0$, is added. The existence and uniqueness of the minimizer $\varphi_{n}$ in $X$ is proved by the following theorem.

Theorem 2.25. For the Hilbert spaces $X$ and $Y$, we assume that $A: X \rightarrow Y$ is a compact linear operator. We also assume that the regularization parameter $\alpha$ is positive. For each $f \in X$ there exist a unique $\varphi_{\alpha} \in X$ such that,

$$
\left\|A \varphi_{\alpha}-f\right\|^{2}+\alpha\left\|\varphi_{\alpha}\right\|^{2}=\inf _{\varphi \in X}\left\{\|A \varphi-f\|^{2}+\alpha\|\varphi\|^{2}\right\} .
$$

The minimizer $\varphi_{\alpha}$ is given by the unique solution of the following equation

$$
\alpha \varphi_{\alpha}+A^{*} A \varphi_{\alpha}=A^{*} f
$$

and depends continuously on $f$.

The right hand side of equation (2.4.4) is known as Tikhonov functional. The Tikhonov regularization scheme is explicitly stated by the following theorem

Theorem 2.26. Let $A: X \rightarrow Y$ be a compact injective linear operator for the Hilbert spaces $X$ and $Y$. Then for each $\alpha>0$ the operator $\alpha I+A^{*} A: X \rightarrow Y$ is a boundedly invertible and the operator

$$
R_{\alpha}:=\left(\alpha I+A^{*} A\right)^{-1} A^{*}
$$

describes a regularization scheme with $\left\|R_{\alpha}\right\| \leq \frac{1}{2 \sqrt{\alpha}}$. 
Proof. See [22]

The Tikhonov regularization replaces the solution of (2.4.1) by the solution of the approximated equation

$$
\alpha \varphi_{\alpha}+A^{*} A \varphi_{\alpha}=A^{*} f
$$

which can be obtained from (2.4.1) by the multiplication with $A^{*}$ and then thr addition of the penalty term $\alpha \varphi_{\alpha}$.

\subsection{Nyström's Method}

In 1930 Nyström's introduced a numerical method to work out the integral equations. A detailed description of the Nyström's Method can be found in [4], [21], [22].

Definition 2.27. Let $\Omega \subset \mathbf{R}^{m}$ be a nonempty compact and Jordan measurable set. Then the sequence of quadratures rules for the integral,

$$
Q(g):=\int_{\Omega} w(x) g(x) d x
$$

is defined as

$$
Q_{n}(g):=\sum_{j=1}^{n} \alpha_{j}^{(n)} g\left(x_{j}^{(n)}\right) .
$$

Here $w(x)$ is some weight function and $x_{1}^{(n)}, x_{2}^{(n)}, \ldots, x_{n}^{(n)}$ are the quadratures points in $\Omega$. The numbers $\alpha_{1}^{(n)}, \alpha_{2}^{(n)}, \ldots, \alpha_{n}^{(n)}$ are called quadrature weights.

Definition 2.28. A sequence $\left(Q_{n}\right)$ of quadrature rule is called convergent if

$$
Q_{n}(g) \rightarrow Q(g), n \rightarrow \infty, \text { for all } g \in C(\Omega) \text {. }
$$

With the help of convergent sequence $\left(Q_{n}\right)$ of quadrature rules, we can approximate the integral operator (2.3.5) with a continuous kernel $K$ as defined in Definition 2.14 by a sequence of numerical integration operators

$$
\left(A_{n} \varphi\right)(x):=\sum_{k=1}^{n} \alpha_{k}^{(n)} K\left(x, x_{k}^{(n)}\right) \varphi\left(x_{k}^{(n)}\right),
$$

for fixed $x \in \Omega$. Here we take $g(\tilde{x})=K(x, \tilde{x}) \varphi(\tilde{x})$ in the integrand of equation (2.5.1). With this the solution to an integral equation of second kind

$$
\varphi-A \varphi=f
$$


is approximated by the solution of

$$
\varphi_{n}-A_{n} \varphi_{n}=f
$$

Using quadrature $Q_{n}$ we discretized the continuous problem into a semi discrete problem, since it is still continuous in the first argument of the Kernel $K$. Therefore equation (2.5.4) is the semi discrete equation. Now with the help of following result (see also [22]), the solution of this semi discrete problem is obtained by solving the finite dimensional system of linear equations.

Theorem 2.29. Let $\varphi_{n}$ be a solution of

$$
\varphi_{n}(x)-\sum_{k=1}^{n} \alpha_{k} K\left(x, x_{k}\right) \varphi_{n}\left(x_{k}\right)=f(x), \quad x \in \Omega .
$$

Then the values $\varphi_{j}^{(n)}=\varphi_{n}\left(x_{j}\right), j=1, \ldots, n$, at the quadratures points satisfy the linear system

$$
\varphi_{j}^{(n)}-\sum_{k=1}^{n} \alpha_{k} K\left(x_{j}, x_{k}\right) \varphi_{k}^{(n)}=f\left(x_{j}\right), \quad j=1, \ldots n .
$$

Conversely, let $\varphi_{j}^{(n)}, j=1, \ldots, n$, be a solution of the system (2.5.6). Then the function $\varphi_{n}$ defined by

$$
\varphi_{n}(x):=f(x)+\sum_{k=1}^{n} \alpha_{k} K\left(x, x_{k}\right) \varphi_{k}^{(n)}, \quad x \in \Omega,
$$

solves equation (2.5.5).

Proof. A proof is worked out in Theorem 12.7 in [22].

Thus with this result we are able to find out the solution of semi discrete equation (2.5.4). Now the basic question arises that if $\varphi$ is the solution of integral equation of second kind (2.5.3) then is there any solution $\varphi_{n}$ of the corresponding approximate integral equation. Also whether the solution $\varphi_{n}$ of the semi discrete equation (2.5.4) converges to the true solution of (2.5.3) or not. For the existence of the solution $\varphi_{n}$ we will use the well known Riesz theory. To implement the Theorem 2.17 we must have the injectivity of the operator $I-A_{n}$. For this we need some properties of the discretized version of the operator $A$ (for more details see [17]).

Definition 2.30 (Consistency). Let $X$ be a normed space. Then the discretization of the operator $A: X \rightarrow X$, i.e., $A_{n}, n \in \mathbb{N}$ is called consistent in $X$ if

$$
\lim _{n \rightarrow \infty}\left\|A \varphi-A_{n} \varphi\right\|=0 \text { for all } \varphi \in X .
$$


Definition 2.31 (Stability). The discretization $A_{n}, n \in \mathbb{N}$ of the operator $A: X \rightarrow$ $X$ is called stable if there exist a constant $C$ and an integer $n_{0} \in \mathbb{N}$ such that the inverse operator $\left(I-A_{n}\right)^{-1}$ exists and uniformly bounded

$$
\left\|\left(I-A_{n}\right)^{-1}\right\| \leq C \text { for all } n>n_{0} .
$$

With these two definition we are in a position to prove the injectivity of the operator $I-A_{n}$.

Lemma 2.32. Assume that the discretization $A_{n}$ of the operator $A: X \rightarrow X$ is stable and consistent then the operator $I-A$ is injective.

Proof. If the inequality

$$
\beta\|\varphi\| \leq\|(I-A) \varphi\| \quad \beta>0,
$$

for all $\varphi \in X$ holds then the operator $I-A$ is injective. On contrary basis we assume that the inequality (2.5.10) is not true, i.e., there exist two sequences $\varphi_{n} \in X$ with $\left\|\varphi_{n}\right\|=1$ and $\phi_{n}:=(I-A) \varphi_{n}$ with $\left\|\phi_{n}\right\| \leq 1 / n$. Due to consistency we have $A_{m} \varphi_{n} \rightarrow A \varphi_{n}, m \rightarrow \infty$ for each fixed $n$. Consequently there exist an index $m=m(n)$ such that

$$
\left\|A_{m} \varphi_{n}-A \varphi_{n}\right\| \leq 1 / n
$$

Now define another sequence

$$
\begin{aligned}
\psi_{n} & :=\left(I-A_{m}\right) \varphi_{n} \\
& =\varphi_{n}-A \varphi_{n}+A \varphi_{n}-A_{m} \varphi_{n} \\
& =\phi_{n}-\left(A_{m}-A\right) \varphi_{n} .
\end{aligned}
$$

This implies that

$$
\begin{aligned}
\left\|\psi_{n}\right\| & =\left\|\phi_{n}-\left(A_{m}-A\right) \varphi_{n}\right\| \\
& \leq\left\|\phi_{n}\right\|+\left\|\left(A_{m}-A\right) \varphi_{n}\right\| \leq 2 / n .
\end{aligned}
$$

Now rewriting the equation (2.5.11) in terms of $\varphi_{n}$, i.e.,

$$
\varphi_{n}=\left(I-A_{m}\right)^{-1} \psi_{n} .
$$

As the operator $A_{m}$ is stable and with the help of equation (2.5.9) we have

$$
1=\left\|\varphi_{n}\right\|=\left\|\left(I-A_{m}\right)^{-1} \psi_{n}\right\| \leq C\left\|\psi_{n}\right\| \leq 2 C / n .
$$

This is a contradiction and the proof is complete.

Since the operator $I-A$ is injective from Theorem 2.17 and Corollary 2.18 we have the unique solvability of the continuous problem (2.5.3). Now coming to the 
next question that the solution $\varphi_{n}$ of the semi discrete equation converges to the true solution $\varphi$. Using equations (2.5.3) and (2.5.4) we have tp rewrite the equation (2.5.3) as

$$
(I-A) \varphi=f .
$$

Adding the term $A_{n} \varphi_{n}$ in both sides of the above equation and then subtract it from the equation (2.5.4) we obtain

$$
\varphi_{n}-\varphi=(I-A)^{-1}\left(A_{n}-A\right) \varphi .
$$

For $n \geq \mathbb{N}$ and with the help of equation (2.5.9) we derive

$$
\left\|\varphi_{n}-\varphi\right\| \leq C\left\|\left(A_{n}-A\right) \varphi\right\|,
$$

which tends to zero due to the equation (2.5.8). We summarize the results in the following theorem.

Theorem 2.33. Assume that we have consistent and stable discretization of equation (2.5.4). Then equation (2.5.3) is solvable in $C(\Omega)$ and the discretization is convergent towards (2.5.3), i.e., there exist an integer $N$ such that equation (2.5.4) is uniquely solvable for every $f \in C(\Omega)$ and for all $n \geq N, \epsilon>0$ the following estimate

$$
\left\|\varphi_{n}-\varphi\right\| \leq \epsilon
$$

holds.

To the end of this section we exhibit the convergence of the discretization (2.5.4) of Nyström's Method by the following theorem.

Theorem 2.34. The sequence $\left(A_{n}\right)$ is collectively compact and pointwise convergent, i.e.,

$$
A_{n} \varphi \rightarrow A \varphi, \quad n \rightarrow \infty \text { for all } \varphi \in C(\Omega)
$$

provided that the quadrature formulas $Q_{n}$ are convergent.

Proof. See Theorem 12.8 in [22]. 


\section{Chapter 3}

\section{Direct Problem of Oseen Flow}

In this chapter we analyze direct problems for the Oseen equation. We present a weak formulation of the Oseen equation, fundamental solution to the Oseen equation and its derivation. We use the boundary integral equation approach to prove existence of the solution to the exterior and interior Dirichlet boundary value problems for the Oseen equation.

\subsection{Oseen Equation}

As a model problem in two dimensions, the steady Oseen equation is derived from the Navier-Stokes equation by linearizing its convective term around a constant velocity $u_{0}=(1,0)$. The details are in the appendix. The Oseen equation is

$$
\mu \triangle u-\partial_{1} u-\nabla p=0, \quad \nabla \cdot u=0 .
$$

Let $D \subset \mathbb{R}^{2}$ be a sufficiently smooth bounded domain with connected exterior. Here, $D$ might possibly have several separate components. Without loss of generality we assume that the origin is contained in $D$. We consider the exterior Dirichlet problem for the Oseen equation (3.1.1) subject to the boundary condition

$$
u=f, \quad \text { on } \quad \partial D,
$$

with a given vector field $f$ and the condition at infinity,

$$
\lim _{x \rightarrow \infty} u(\hat{x})=u_{\infty}, \quad \hat{x}=\frac{x}{|x|},
$$

in the sense of uniform convergence with respect to all directions $\hat{x} \in \mathbb{S}$, where $u_{\infty}$ is a constant vector. For homogeneous boundary values $f=0$ and $u_{\infty}=u_{0}=(1,0)^{T}$, this boundary value problem models a two-dimensional flow around a cylindrical obstacle with cross section $D$ immersed in a fluid with constant velocity $u_{\infty}$. In Fig. 3.1 we exhibit the flow field visualization of this boundary value problem. 


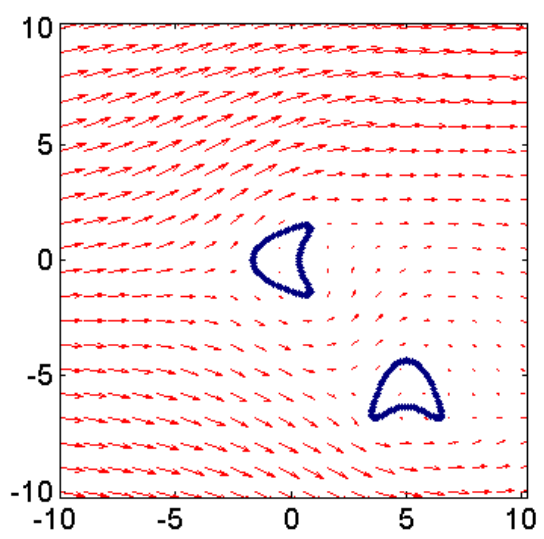

Figure 3.1: We show a visualization of the flow field $u$ of the Oseen equation around two obstacles.

Often, a variational form of the Oseen equation is employed in computational fluid dynamics (CFD) literature [16]. Multiplying the Oseen equation with a test vector field $v \in C_{0}^{1}\left(\mathbb{R}^{2} \backslash \bar{D}, \mathbb{R}^{2}\right)$ and the equation of continuity with a scaler field $q \in C_{0}^{1}\left(\mathbb{R}^{2} \backslash \bar{D}, \mathbb{R}\right)$ and then using the Gauss divergence theorem [22] and partial integration we derive the following weak formulation of Oseen equation

$$
\begin{aligned}
a(u, v)+d(p, v) & =0, \\
d(q, u) & =0, \\
\lim _{R \rightarrow \infty} \frac{1}{R} \int_{|x|=R}\left|u-u_{\infty}\right| d s(x) & =0 .
\end{aligned}
$$

Here $a$ and $d$ are defined as the bilinear forms

$$
\begin{aligned}
a(u, v) & :=\int_{\mathbb{R}^{2} \backslash \bar{D}}\left\{\mu \nabla v: \nabla u-u \cdot \partial_{1} v\right\} d x, \\
d(p, v) & :=-\int_{\mathbb{R}^{2} \backslash \bar{D}} p \nabla \cdot v d x .
\end{aligned}
$$

Then for a given field $f \in W^{\frac{1}{2}}\left(\partial D, \mathbb{R}^{2}\right)$ we say that the pair

$$
(u, p) \in W_{l o c}^{1}\left(\mathbb{R}^{2} \backslash D, \mathbb{R}^{2}\right) \times L_{l o c}^{2}\left(\mathbb{R}^{2} \backslash D, \mathbb{R}\right)
$$

is the weak solution to the Dirichlet problem (3.1.1)-(3.1.3) if $\left.u\right|_{\partial D}=f$ in the sense of the trace operator and (3.1.4)-(3.1.6) are satisfied.

We will also need the adjoint Oseen equation

$$
\mu \triangle \tilde{u}+\partial_{1} \tilde{u}-\nabla \tilde{p}=0, \quad \operatorname{div} \tilde{u}=0 .
$$


It coincides with the Oseen equation (3.1.1) when $u_{0}=(-1,0)^{T}$. Thus solutions to the adjoint Oseen equation are also solutions to an Oseen equation and all statements about such solutions carry over to the adjoint equation.

Since we use the method of boundary integral equation to solve the equation (3.1.4)-(3.1.6) with a layer potential approach. For this we need to understand the fundamental solution of Oseen equation that we derive as follows.

\subsubsection{Derivation of the Fundamental Solution}

In the following, we basically follow Finn [13], Galdi [16] or Oseen [33] to obtain the fundamental solution of the Oseen equation. Since the choice of the constants and the signs of the terms varies from paper to paper and it is slightly delicate, here we carefully worked out the arguments. We denote as the tensor field $E$ and the vector field $e$, defined by

$$
\begin{gathered}
E_{i j}(y)=E_{i j}\left(y_{1}, y_{2}\right)=\left(\frac{\partial^{2}}{\partial y_{i} \partial y_{j}}-\delta_{i j} \Delta\right) \Phi\left(y_{1}, y_{2}\right), \\
e(y)=e_{j}\left(y_{1}, y_{2}\right)=\frac{\partial}{\partial y_{j}}\left(\mu \Delta-\frac{\partial}{\partial y_{1}}\right) \Phi\left(y_{1}, y_{2}\right),
\end{gathered}
$$

for $i, j=1,2$. Here $\Phi$ is a smooth real function for $0 \neq y \in \mathbb{R}^{2}$. Multiplying equation (3.1.11) with the operator $\mu \Delta-\frac{\partial}{\partial y_{1}}$ and taking derivative of equation(3.1.12) w.r.t. $\frac{\partial}{\partial y_{i}}$, and then subtracting we have

$$
\left(\mu \Delta-\frac{\partial}{\partial y_{1}}\right) E_{i j}\left(y_{1}, y_{2}\right)-\frac{\partial e_{j}\left(y_{1}, y_{2}\right)}{\partial y_{j}}=-\delta_{i j} \Delta\left(\mu \Delta-\frac{\partial}{\partial y_{1}}\right) \Phi\left(y_{1}, y_{2}\right) .
$$

Using Einstein's convention we also have

$$
\frac{\partial}{\partial y_{l}} E_{l j}\left(y_{1}, y_{2}\right)=0
$$

$E$ and $e$ are the solutions of equations (3.1.13) and (3.1.14) if and only if the function $\Phi$ is the fundamental solution of the linear partial differential operator given in the right hand side of equation (3.1.13). Following Definition 2.12, we set Dirac's delta function is equal to $\Delta \mathcal{E}(|y|)$, here $\mathcal{E}(|y|)$ is the fundamental solution of the Laplace equation. Thus we have,

$$
\Delta\left(\mu \Delta-\frac{\partial}{\partial y_{1}}\right) \Phi\left(y_{1}, y_{2}\right)=\Delta \mathcal{E}(|y|)
$$

Now, we try to obtain the solution of above equation (3.1.15) into the form

$$
\Phi\left(y_{1}, y_{2}\right)=\int^{y_{1}}\left[\Phi_{1}\left(\eta, y_{2}\right)-\Phi_{2}\left(\eta, y_{2}\right)\right] d \eta
$$


with $\Phi_{1}$ and $\Phi_{2}$ to be selected appropriately. After taking derivative of above equation w.r.t. $y_{1}$, the above equation reduces to

$$
\frac{\partial \Phi\left(y_{1}, y_{2}\right)}{\partial y_{1}}=\left[\Phi_{1}\left(y_{1}, y_{2}\right)-\Phi_{2}\left(y_{1}, y_{2}\right)\right]
$$

Furthermore, taking the derivative of equation (3.1.15) with respect to $y_{1}$ and then substituting the value of (3.1.17) in it, we obtain

$$
\Delta\left(\mu \Delta-\frac{\partial}{\partial y_{1}}\right)\left[\Phi_{1}\left(y_{1}, y_{2}\right)-\Phi_{2}\left(y_{1}, y_{2}\right)\right)=\Delta\left(\frac{\partial}{\partial y_{1}} \mathcal{E}(|y|)\right) .
$$

The following choice of the function $\Phi_{2}$,

$$
\Phi_{2}\left(y_{1}, y_{2}\right)=\mathcal{E}(|y|)
$$

leads equation (3.1.18) into the form

$$
\Delta\left[-\mu \Delta \mathcal{E}(|y|)+\left(\mu \Delta-\frac{\partial}{\partial y_{1}}\right) \Phi_{1}\left(y_{1}, y_{2}\right)\right]=0 .
$$

For $\Phi_{1}\left(y_{1}, y_{2}\right)$ to be the solution of equation (3.1.18), it is sufficient to have

$$
\left(\Delta-\frac{1}{\mu} \frac{\partial}{\partial y_{1}}\right) \Phi_{1}\left(y_{1}, y_{2}\right)=\Delta \mathcal{E}(|y|) .
$$

Setting

$$
\Phi_{1}\left(y_{1}, y_{2}\right)=\frac{e^{\lambda y_{1}}}{|y|^{(n-2) / 2}} f(\lambda|y|)
$$

with $\lambda=\frac{1}{2} \mu$. By a direct calculation and taking $\lambda|x-y|=\xi$, we obtain,

$$
\left(\Delta-\frac{1}{\mu} \frac{\partial}{\partial y_{1}}\right) \Phi_{1}\left(y_{1}, y_{2}\right)=\frac{e^{\lambda y_{1}}}{|y|^{(n+2) / 2}}\left\{\xi^{2} f^{\prime \prime}(\xi)+\xi f^{\prime}(\xi)-\xi^{2} f(\xi)\right\},
$$

with

$$
f^{\prime}(\xi):=\frac{\partial}{\partial \xi} f(\xi)
$$

The equation

$$
\xi^{2} f^{\prime \prime}(\xi)+\xi f^{\prime}(\xi)-\xi^{2} f(\xi)=0,
$$

is the modified Bessel equation and it has two independent solutions

$$
\begin{aligned}
I_{0}(\xi) & =\sum_{m=0}^{\infty} \frac{\left(\frac{1}{2} \xi\right)^{2 m}}{(m !)^{2}} \\
K_{0}(\xi) & =-I_{0}(\xi) \log \frac{1}{2} \xi+\sum_{m=0}^{\infty} \frac{\left(\frac{1}{2} \xi\right)^{2 m}}{(m !)^{2}} \Theta(m+1),
\end{aligned}
$$


which are known as modified Bessel functions of first and second kind, respectively. Here $\Theta(n)$ is a digamma function given by

$$
\Theta(n)=-\varsigma+\sum_{k=1}^{n-1} \frac{1}{k}
$$

where $\varsigma$ is Euler-Mascheroni constant. As $I_{0}(\xi)$ is smooth for all values of $\xi$ while $K_{0}(\xi)$ is singular at $\xi=0$, we can write $K_{0}(\xi)$ explicitly as

$$
K_{0}(\xi)=\log \frac{1}{\xi}-\varsigma+\mathcal{X}(\xi) .
$$

Here

$$
\mathcal{X}(\xi)=-\sum_{m=1}^{\infty} \frac{\left(\frac{1}{2} z\right)^{2 m}}{m ! \Gamma(m)}\left\{\ln \frac{1}{2} \xi-\Theta(m+1)\right\},
$$

with very useful properties (see [16])

$$
\mathcal{X}(\xi)=o(1), \quad \frac{d^{k} \mathcal{X}(\xi)}{d \xi^{k}}=o\left(\xi^{-k}\right) \text { as } \xi \rightarrow 0 .
$$

Equation (3.1.20) tells us that if $\Phi_{1}\left(y_{1}, y_{2}\right)$ is its solution, then it must take the form of fundamental solution of Laplace equation in the neighborhood of $|y|=0$. Thus from the fundamental solution of Laplace equation and in the view of (3.1.20) and (3.1.22) we are bound to take the following function

$$
\Phi_{1}\left(y_{1}, y_{2}\right)=-\frac{1}{2 \pi} K_{0}\left(\frac{|y|}{2 \mu}\right) e^{y_{1} / 2 \mu}
$$

Also for two dimensional space the equation (3.1.19) takes the following form

$$
\left.\Phi_{2}\left(y_{1}, y_{2}\right)=\frac{1}{2 \pi} \ln |y|\right) .
$$

Thus we have the function $\Phi\left(y_{1}, y_{2}\right)$, where $\Phi_{1}\left(y_{1}, y_{2}\right)$ and $\Phi_{2}\left(y_{1}, y_{2}\right)$ are described above,

$$
\Phi\left(y_{1}, y_{2}\right)=\int_{0}^{y_{1}}\left[\Phi_{2}\left(\eta, y_{2}\right)-\Phi_{1}\left(\eta, y_{2}\right)\right] d \eta+\Phi_{0}\left(y_{2}\right)
$$

Here $\Phi_{0}\left(y_{2}\right)$ is the function of $y_{2}$ only and it is to be fixed appropriately. Now our next task is to find $\Phi_{0}\left(y_{2}\right)$, from equation(3.1.19) and (3.1.20), we have

$$
\frac{\partial^{2}}{\partial y_{2}^{2}}\left(\Phi_{2}-\Phi_{1}\right)=-\frac{\partial}{\partial y_{1}}\left(\frac{\partial}{\partial y_{1}}\left(\Phi_{2}-\Phi_{1}\right)+\frac{1}{\mu} \Phi_{1}\right)
$$


Now taking double derivative of (3.1.27) with respect to the second argument and then using the value of equation (3.1.28) we have

$$
\begin{aligned}
\frac{\partial^{2} \Phi\left(y_{1}, y_{2}\right)}{\partial y_{2}^{2}}= & -\frac{\partial}{\partial y_{1}}\left[\Phi_{2}\left(y_{1}, y_{2}\right)-\Phi_{1}\left(y_{1}, y_{2}\right)\right]-\frac{1}{\mu} \Phi_{1}\left(y_{1}, y_{2}\right) \\
& -\frac{1}{\mu} \Phi_{1}\left(0, y_{2}\right)+\frac{\partial^{2}}{\partial y_{2}^{2}} \Phi_{0}\left(y_{2}\right) .
\end{aligned}
$$

Since $\Phi_{1}\left(0, y_{2}\right)=-1 / 2 \pi K_{0}\left(\frac{\left|y_{2}\right|}{2 \mu}\right)$ and this diverges logarithmically fast when $y_{2} \rightarrow$ 0 , while the other terms remain bounded. Due to this factor we face singularity in the fundamental solution $E_{22}$. The best possible way to avoid this singularity is to choose

$$
\frac{\partial^{2}}{\partial y_{2}^{2}} \Phi_{0}\left(y_{2}\right)=\frac{1}{2 \pi \mu} K_{0}\left(\frac{\left|y_{2}\right|}{2 \mu}\right)
$$

Under the condition $\Phi_{0}^{\prime}(0)=\Phi_{0}(0)=0$, above equation reduces to

$$
\Phi_{0}\left(y_{2}\right)=\frac{1}{2 \pi \mu} \int_{0}^{y_{2}}\left(y_{2}-\eta\right) K_{0}\left(\frac{|\eta|}{2 \mu}\right) d \eta
$$

By substituting the value of $\Phi_{0}\left(y_{2}\right)$ in (3.1.27) we have the final expression for the function $\Phi\left(y_{1}, y_{2}\right)$, i.e.,

$$
\begin{aligned}
\Phi\left(y_{1}, y_{2}\right)= & \frac{1}{4 \pi \mu} \int_{0}^{y_{1}}\left[\log \sqrt{\eta^{2}+y_{2}^{2}}+K_{0}\left(\frac{1}{2 \mu} \sqrt{\eta^{2}+y_{2}^{2}}\right)\right] d \eta \\
& -\frac{1}{2 \pi} \int_{0}^{y_{2}}\left(y_{2}-\eta\right) K_{0}(\lambda|\eta|) d \eta .
\end{aligned}
$$

Finally substituting the values of $\Phi\left(y_{1}, y_{2}\right)$ in the equation (3.1.11), we have

$$
E\left(y_{1}, y_{2}\right)=\left(\begin{array}{cc}
\Psi\left(y_{1}, y_{2}\right)-\Psi_{1}\left(y_{1}, y_{2}\right) & -\Psi_{2}\left(y_{1}, y_{2}\right) \\
-\Psi_{2}\left(y_{1}, y_{2}\right) & \Psi\left(y_{1}, y_{2}\right)+\Psi_{1}\left(y_{1}, y_{2}\right)
\end{array}\right)
$$

with

$$
\begin{aligned}
\Psi\left(y_{1}, y_{2}\right) & =\frac{1}{4 \pi \mu} e^{y_{1} / 2 \mu} K_{0}\left(\frac{|y|}{2 \mu}\right) \\
\Psi_{i}\left(y_{1}, y_{2}\right) & =\frac{y_{i}}{2 \pi|y|}\left[\frac{1}{|y|}-\frac{1}{2 \mu} e^{y_{1} / 2 \mu} K_{1}\left(\frac{|y|}{2 \mu}\right)\right], \quad \text { for } \quad i=1,2 .
\end{aligned}
$$

Here $K_{0}$ and $K_{1}$ are the modified Bessel functions [45].

Also we can evaluate the pressure term $e$ by substituting the value of equation (3.1.15) in equation (3.1.12), we have

$$
e_{j}\left(y_{1}, y_{2}\right)=\frac{\partial}{\partial y_{j}} \mathcal{E}(|y|),
$$


or

$$
e\left(y_{1}, y_{2}\right)=\operatorname{grad} \Phi\left(y_{1}, y_{2}\right), \quad \text { with } \quad \Phi\left(y_{1}, y_{2}\right)=\frac{1}{2 \pi} \ln |y| .
$$

With the help of power series expansion of the Bessel functions $K_{0}$ and $K_{1}$, a straight forward calculation divides the fundamental solution of the Oseen equation in the following way

$$
E\left(y_{1}, y_{2}\right)=E_{0}\left(y_{1}, y_{2}\right)+\ln |y| A\left(y_{1}, y_{2}\right)+B\left(y_{1}, y_{2}\right)
$$

where

$$
E_{0}\left(y_{1}, y_{2}\right)=\frac{1}{4 \pi \mu}\left(-\ln |y| I+\frac{y y^{T}}{|y|^{2}}\right),
$$

with $I$ is the identity matrix and $A=A_{i j}, B=B_{i j}$ are analytic matrices with

$$
\begin{gathered}
A_{i j}=-\frac{1}{4 \pi \mu}\left\{\delta_{i j}\left(-1+e^{y_{1} / 2 \mu} I_{0}\left(\frac{|y|}{2 \mu}\right)\right)(-1)^{i+j} \frac{y_{k}}{4 \mu} e^{y_{2} / 2 \mu} \sum_{m=0}^{\infty} \frac{\left(|y|^{2} / 16 \mu^{2}\right)^{m}}{m !(m+1) !}\right\} \\
k=1 \text { for } i=j, k=2 \text { for } i \neq j
\end{gathered}
$$

Also

$$
\begin{gathered}
L=\frac{1}{4 \pi \mu}\left\{(-\gamma+\ln |4 \mu|) I_{0}\left(\frac{|y|}{2 \mu}\right)+\left[\frac{|y|^{2}}{4(2 \mu)^{2}}+\left(1+\frac{1}{2}\right) \frac{|y|^{2}}{\left(4(2 \mu)^{2}\right)^{2}}+\ldots\right]\right\}, \\
M_{k}=\frac{y_{k}}{16 \pi \mu} \sum_{m=0}^{\infty}\left\{\ln |4 \mu|+\frac{1}{2}\{\Theta(m+1)+\Theta(m+2)\}\right\} \frac{\left[|y|^{2} / 16 \mu^{2}\right]^{m}}{m !(m+1) !}-\frac{y_{k}}{2 \pi|y|^{2}} e^{y_{1} / 2 \mu},
\end{gathered}
$$

and

$$
N=\frac{y_{1}}{4 \pi \mu\left|y^{2}\right|}\left\{y_{1}+2 \mu\right\} .
$$

From (3.1.34) we observe that $E_{0}\left(y_{1}, y_{2}\right)$ is the fundamental solution of Stokes equation (2.2.10).

For several applications we need to observe the asymptotic behavior of the fundamental solution of Oseen equation.

Theorem 3.1. The fundamental solution (3.1.32) of the Oseen equation admits the asymptotics

$$
\begin{aligned}
E(y)= & \frac{1}{2 \pi|y|^{2}}\left(\begin{array}{cc}
-y_{1} & -y_{2} \\
-y_{2} & y_{1}
\end{array}\right) \\
& +\frac{e^{\left(y_{1}-|y|\right) / 2 \mu}}{4 \sqrt{\pi \mu|y|^{3}}}\left(\begin{array}{cc}
|y|+y_{1} & y_{2} \\
y_{2} & |y|-y_{1}
\end{array}\right)\left[1++O\left(\frac{1}{|y|}\right)\right]
\end{aligned}
$$

for $|y| \rightarrow \infty$. 
Proof. For the proof, we use the asymptotics of the modified Bessel functions. As the asymptotes of the modified Bessel functions, described in [1], with $|\arg \xi|<\frac{3}{2}|\pi|$ are

$$
\begin{aligned}
& K_{0}(\xi)=\sqrt{\frac{\pi}{2 \mu}} e^{-\xi}\left\{1-\frac{1}{8 \xi}+\frac{9}{2 !(8 \xi)^{2}} \ldots\right\}, \\
& K_{1}(\xi)=\sqrt{\frac{\pi}{2 \mu}} e^{-\xi}\left\{1+\frac{3}{8 \xi}+\frac{2.6}{2 !(8 \xi)^{2}} \ldots\right\},
\end{aligned}
$$

$|\xi| \rightarrow \infty$. With the help of above values, we calculate the

$$
\begin{aligned}
\Psi(y)-\Psi_{1}(y) & =-\frac{y_{1}}{2 \pi|y|^{2}}+\frac{|y|+y_{1}}{4 \sqrt{\pi \mu|y|^{3}}} e^{\left(y_{1}-|y|\right) / 2 \mu}\left\{1+O\left(\frac{1}{|y|}\right)\right\}, \\
-\Psi_{2}(y) & =-\frac{y_{2}}{2 \pi|y|^{2}}+\frac{y_{2}}{4 \sqrt{\pi \mu|y|^{3}}} e^{\left(y_{1}-|y|\right) / 2 \mu}\left\{1+O\left(\frac{1}{|y|}\right)\right\}, \\
\Psi(y)+\Psi_{1}(y) & =\frac{y_{1}}{2 \pi|y|^{2}}+\frac{|y|-y_{1}}{4 \sqrt{\pi \mu|y|^{3}}} e^{\left(y_{1}-|y|\right) / 2 \mu}\left\{1+O\left(\frac{1}{|x|}\right)\right\} .
\end{aligned}
$$

Thus from equation (3.1.32) we proved the asymptotics (3.1.36), which are uniform in all direction when $|y| \rightarrow \infty$.

\subsection{Direct Problem}

In this section we presents the direct problem of the Oseen equation. We use the integral equation approach for the interior and exterior Oseen problem. We start this section by introducing the single layer potentials operators for the Oseen equation and using standard tools of integral equations we presents the weak solutions of the interior and exterior Dirichlet problem (3.1.1)-(3.1.3) of Oseene equation. For certain reasons we also introduce the adjoint Oseen equation.

Definition 3.2. Let $\partial D$ be of class $C^{2}$ and $\varphi$ be a vector field on $\partial D$, then the single layer potential is defined by the pair

$$
(\mathscr{S} \varphi)(x):=\int_{\partial D} E(x-y) \varphi(y) d s(y), \quad(\mathscr{P} \varphi)(x):=\int_{\partial D} e(x-y) \cdot \varphi(y) d s(y),
$$

for $x \in \mathbb{R}^{2} \backslash \partial D$. E and e are given by equations (3.1.32) and (3.1.33).

From the above definition and the fundamental solution of Oseen equation, it is observed that for an integrable density $\varphi$ the pair of single layer potential operator $(\mathscr{S} \varphi, \mathscr{P} \varphi)$ solves the Oseen equation both in $D$ and $\mathbb{R}^{2} \backslash \bar{D}$.

To investigate the properties of the pair of single layer potential operator on the boundary $\partial D$, we need the jump relations. From equation (3.1.34), it is observed 
that the fundamental solution of Oseen equation and the fundamental solution of Stokes equation has the same singular behavior at $x=0$ because of $A(0)=0$. Thus the jump relations for the Oseen equation are similar to the jump relations of the Stokes equation. In the following theorem we have a brief sketch of jump relations for the Oseen equation.

Theorem 3.3. Let $\partial D$ be of class $C^{2}$. Let $\nu$ be the outward unit normal on the obstacle $D$. Then for the single layer potential operators $\mathscr{S} \varphi$ and $\mathscr{P} \varphi$, we have

$$
\begin{aligned}
\lim _{h \rightarrow+0}(\mathscr{S} \varphi)(x \pm h \nu(x))= & \int_{\partial D} E(x-y) \varphi(y) d s(y) \\
\lim _{h \rightarrow+0}(\mathscr{P} \varphi)(x \pm h \nu(x))= & \int_{\partial D} e(x-y) \cdot \varphi(y) d s(y) \pm \frac{1}{2} \nu(x) \cdot \varphi(x)(3.2) \\
\lim _{h \rightarrow+0} \frac{\partial(\mathscr{S} \varphi)}{\partial \nu(x)}(x \pm h \nu(x))= & \int_{\partial D} \frac{\partial E(x-y)}{\partial \nu(x)} \varphi(y) d s(y) \\
& \mp \frac{1}{2 \mu}[\varphi(x)-\varphi(x) \cdot \nu(x) \nu(x)]
\end{aligned}
$$

for $x \in \partial D$.

Proof. Compare [30], Theorem 4.2.

Theorem 3.4. The single layer potential operator is a bounded operator

$$
\mathscr{S}:\left(W^{r}\left(\partial D, \mathbb{R}^{2}\right)\right)^{2 \times 1} \rightarrow\left(W^{r+1}\left(\partial D, \mathbb{R}^{2}\right)\right)^{2 \times 1}
$$

for all $r \in \mathbb{R}$.

Proof. For the proof we follow Kress and Meyer [23] who used the idea of isomorphism. We know that the Sobolev spaces $W^{r}\left(\partial D, \mathbb{R}^{2}\right)$ and the spaces $W^{r}\left([0,2 \pi], \mathbb{R}^{2}\right)$ are isomorphic to each other (see Theorem 8.14 of [22]). Thus if we show the boundedness of the parametrized form of the operator $(\mathscr{S} \varphi)(x)$, then the proof is completed. To construct the isomorphic Sobolev space first we parametrize our boundary $\partial D$ with $2 \pi$ period such that $\partial D=\{z(t): t \in[0,2 \pi]\}$. Then from equation (3.1.34) the parametrized form of the single layer potential operator is given by

$$
(\mathbb{S} \psi)(t)=\int_{0}^{2 \pi}\left\{\ln \left(\frac{4}{e} \sin ^{2} \frac{t-\tau}{2}\right) M_{1}(t, \tau)+M_{2}(t, \tau)\right\} \psi(\tau) d \tau, t \in[0,2 \pi],
$$

for $\psi=\varphi(z(t))\left|z^{\prime}(t)\right|$. Here, $M_{1}$ and $M_{2}$ are twice differentiable matrices with

$$
M_{1}(t, t)=-\frac{1}{2 \pi \mu} I, \quad 0 \leq t \leq 2 \pi .
$$

With the help of Theorem 12.15 of [22] we are in the position to say that the operator $(\mathbb{S} \psi): W^{r}\left([0,2 \pi], \mathbb{R}^{2}\right) \rightarrow W^{r+1}\left([0,2 \pi], \mathbb{R}^{2}\right)$ is bounded for all $r \geq 0$. Now by the 
duality principle we can extend this argument for all $r \in \mathbb{R}$. Finally, from the isomorphism of the Sobolev spaces, statement of the theorem is proven.

The last Theorem 3.4 confirms that the single layer potential operator $\mathscr{S}$ is bounded on the boundary. However if we extend this operator to interior or exterior of the domain $D$ then a simple question arises that whether is it still bounded or not. Following Kress and Meyer [23], we have the following result which provide the boundedness of the single layer potential operator $\mathscr{S}$ in interior or exterior of the domain $D$.

Theorem 3.5. The single layer potential operator $\mathscr{S}$ is a bounded operator

$$
\mathscr{S}: W^{-1 / 2}\left(\partial D, \mathbb{R}^{2}\right) \rightarrow W^{1}\left(D, \mathbb{R}^{2}\right), \quad \mathscr{S}: W^{-1 / 2}\left(\partial D, \mathbb{R}^{2}\right) \rightarrow W^{1}\left(D_{R}, \mathbb{R}^{2}\right),
$$

and $\mathscr{P}$ is a bounded operator from

$$
\mathscr{P}: W^{-1 / 2}\left(\partial D, \mathbb{R}^{2}\right) \rightarrow L^{2}\left(D, \mathbb{R}^{2}\right), \quad \mathscr{P}: W^{-1 / 2}\left(\partial D, \mathbb{R}^{2}\right) \rightarrow L^{2}\left(D_{R}, \mathbb{R}^{2}\right),
$$

where $D_{R}:=\left\{x \in \mathbb{R}^{2} \backslash \bar{D}:|x| \leq R\right\}$.

Proof. Assume an arbitrary bounded domain $B$ with its boundary $\partial B$. Now we assume that the Oseen equation (3.1.1) defined on a bounded domain $B$ with sufficiently smooth boundary and having the exterior normal vector $\nu$. Also consider that the pair $(u, p)$ be the sufficiently smooth solution of the Oseen equation. Now working on equation (3.1.1), we have

$$
\mu u \cdot \Delta u-u \cdot \partial_{1} u-\nabla \cdot(p u)=0 .
$$

Integrating over the domain $B$, we have

$$
\mu \int_{B} u \cdot \Delta u d x-\int_{B} u \cdot \partial_{1} u d x-\int_{B} \nabla \cdot(p u) d x=0 .
$$

Using the Gauss divergence theorem, we obtain

$$
\begin{aligned}
\mu \int_{B} u \cdot \Delta u d x & =\mu \int_{\partial B} u \cdot \frac{\partial u}{\partial \nu} d s-\int_{B}|\nabla u|^{2} d x \\
\int_{B} u \cdot \partial_{1} u d x & =-\int_{B} u \cdot \partial_{1} u d x+\int_{\partial B} u \cdot \nu d s \\
& =\frac{1}{2} \int_{\partial B} u \cdot \nu_{1} u d s \\
\int_{B} \nabla \cdot(p u) d x & =\int_{\partial B} p u d s
\end{aligned}
$$

Substituting the obtained values in equation (3.2.8), we have

$$
\mu \int_{B}|\nabla u|^{2} d x=\int_{\partial B} u \cdot\left(\mu \frac{\partial u}{\partial \nu}-p \nu-\frac{\nu_{1}}{2} u\right) d s .
$$


From the jump relations (3.2.2)-(3.2.4), the above equation holds for the pair of single layer potential operators $(\mathscr{S} \varphi, \mathscr{P} \varphi)$ provided that $\varphi \in C^{0, \alpha}(\partial D)$. Now choosing the domain $B=D$ in equation (3.2.9) we obtain

$$
\begin{aligned}
\mu \int_{D}|\nabla \mathscr{S} \varphi|^{2} d x= & \int_{\partial D} \mathscr{S} \varphi \cdot\left(\mu \frac{\partial \mathscr{S} \varphi}{\partial \nu}-\mathscr{P} \varphi \nu-\frac{\nu_{1}}{2} \mathscr{S} \varphi\right) d s \\
& +\int_{\partial D} \mathscr{S} \varphi \cdot\left(\frac{1}{2}[\varphi-\varphi \cdot \nu \nu]+\frac{1}{2} \varphi \cdot \nu \nu\right) d s .
\end{aligned}
$$

This implies

$$
\begin{aligned}
\mu \int_{D}|\nabla \mathscr{S} \varphi|^{2} d x= & \int_{\partial D} \mathscr{S} \varphi \cdot\left(\mu \frac{\partial \mathscr{S} \varphi}{\partial \nu}-\mathscr{P} \varphi \nu-\frac{\nu_{1}}{2} \mathscr{S} \varphi\right) d s \\
& +\frac{1}{2} \int_{\partial D} \mathscr{S} \varphi \cdot \varphi d s .
\end{aligned}
$$

Similarly now taking $B=D_{R}$ in equation (3.2.9), where $D_{R}:=\left\{x \in \mathbb{R}^{2} \backslash \bar{D}:|x| \leq\right.$ $R\}$, leading to two boundaries $|x|=R$ and $\partial D$.

$$
\begin{aligned}
\mu \int_{D_{R}}|\nabla \mathscr{S} \varphi|^{2} d x= & \int_{|x|=R} \mathscr{S} \varphi \cdot\left(\mu \frac{\partial \mathscr{S} \varphi}{\partial \nu}-\mathscr{P} \varphi \nu-\frac{\nu_{1}}{2} \mathscr{S} \varphi\right) d s \\
& -\int_{\partial D} \mathscr{S} \varphi \cdot\left(\mu \frac{\partial \mathscr{S} \varphi}{\partial \nu}-\mathscr{P} \varphi \nu-\frac{\nu_{1}}{2} \mathscr{S} \varphi\right) d s \\
& -\int_{\partial D} \mathscr{S} \varphi \cdot\left(-\frac{1}{2}[\varphi-\varphi \cdot \nu \nu]-\frac{1}{2} \varphi \cdot \nu \nu\right) d s . \\
\mu \int_{D_{R}}|\nabla \mathscr{S} \varphi|^{2} d x= & -\int_{\partial D} \mathscr{S} \varphi \cdot\left(\mu \frac{\partial \mathscr{S} \varphi}{\partial \nu}-\mathscr{P} \varphi \nu-\frac{\nu_{1}}{2} \mathscr{S} \varphi\right) d s \\
& +\frac{1}{2} \int_{\partial D} \mathscr{S} \varphi \cdot \varphi d s-\mathbb{I}_{R}(\varphi) .
\end{aligned}
$$

with

$$
\mathbb{I}_{R}(\varphi):=\int_{|x|=R} \mathscr{S} \varphi \cdot\left(\mathscr{P} \varphi \nu+\frac{\nu_{1}}{2} \mathscr{S} \varphi-\mu \frac{\partial \mathscr{S} \varphi}{\partial \nu}\right) d s
$$

Adding equation (3.2.11) and equation(3.2.12), we have

$$
\mu \int_{D}|\nabla \mathscr{S} \varphi|^{2} d x+\mu \int_{D_{R}}|\nabla \mathscr{S} \varphi|^{2} d x=\int_{\partial D} \mathscr{S} \varphi \cdot \varphi d s-\mathbb{I}_{R}(\varphi) .
$$

Since 


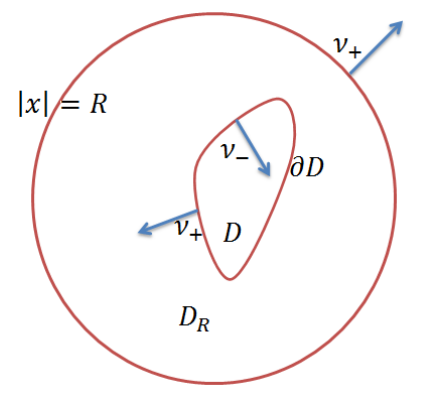

Figure 3.2: Shows that how the jump relations works with two boundaries

$$
\begin{aligned}
\left|\mathbb{I}_{R}(\varphi)\right| & =\left|\int_{|x|=R} \mathscr{S} \varphi \cdot\left(\mathscr{P} \varphi \nu+\frac{\nu_{1}}{2} \mathscr{S} \varphi-\mu \frac{\partial \mathscr{S} \varphi}{\partial \nu}\right) d s\right| \\
& \leq \int_{|x|=R}\left|\mathscr{S} \varphi \cdot\left(\mathscr{P} \varphi \nu+\frac{\nu_{1}}{2} \mathscr{S} \varphi-\mu \frac{\partial \mathscr{S} \varphi}{\partial \nu}\right)\right| d s \\
& \leq \int_{|x|=R}|\mathscr{S} \varphi| \cdot\left(|\mathscr{P} \varphi|+\frac{\nu_{1}}{2}|\mathscr{S} \varphi|+\mu\left|\frac{\partial \mathscr{S} \varphi}{\partial \nu}\right|\right) d s
\end{aligned}
$$

To analyze the above inequality (3.2.15) in more detail we need the asymptotes of the fundamental solution of the Oseen equation.

$$
\begin{aligned}
|E(x)| & \leq \frac{e^{\left(x_{1}-|x|\right) / 2 \mu}}{4 \sqrt{\pi \mu|x|}} \max \left(\frac{|x|+x_{1}+x_{2}}{|x|}, \frac{|x|-x_{1}+x_{2}}{|x|}\right)+O\left(\frac{1}{|x|}\right) \\
& \leq c_{1} \frac{1}{\sqrt{|x|}} e^{\left(x_{1}-|x|\right) / 2 \mu}\left(1+\frac{x_{1}+x_{2}}{|x|}\right)+O\left(\frac{1}{|x|}\right) \\
& \leq c_{1} \frac{1}{\sqrt{|x|}} e^{\left(x_{1}-|x|\right) / 2 \mu}\left(1+O\left(\frac{1}{|x|}\right)\right) \\
& \leq c_{1} \frac{1}{\sqrt{|x|}} e^{\left(x_{1}-|y|\right) / 2 \mu}+O\left(\frac{1}{\sqrt{|x|}}\right)
\end{aligned}
$$

where $c_{1}=1 / 4 \sqrt{\pi \mu}$. We calculate each term of the inequality (3.2.15) separately.

$$
\begin{aligned}
|\mathscr{S} \varphi(x)| & =\left|\int_{\partial D} E(x-y) \varphi(y) d s(y)\right| \\
& \leq \int \partial D|E(x-y)||\varphi(y)| d s(y) \\
& \leq\|\varphi\|_{\infty} \int_{\partial D}|E(x-y)| d s(y) .
\end{aligned}
$$


With the help of inequality (3.2.16), we can write the last inequality as follows

$$
\begin{aligned}
|\mathscr{S} \varphi(x)| & \leq\|\varphi\|_{\infty}\left(c_{1} \frac{1}{\sqrt{|x|}} e^{\left(x_{1}-|x|\right) / 2 \mu}+O\left(\frac{1}{\sqrt{|x|}}\right)\right) \int_{\partial D} d s(y) \\
& \leq \tilde{c_{1}}\|\varphi\|_{\infty} \frac{1}{\sqrt{|x|}} e^{\left(x_{1}-|x|\right) / 2 \mu}+O\left(\frac{1}{\sqrt{|x|}}\right) .
\end{aligned}
$$

Here $\tilde{c_{1}}=c_{1} \int_{\partial D} d s(y)$. Similarly we calculate the other terms of inequality (3.2.15), we obtain,

$$
\begin{aligned}
\left|\frac{\partial \mathscr{S} \varphi}{\partial \nu(x)}(x)\right| & =|\nu(x) \cdot \nabla \mathscr{S} \varphi(x)| \\
& \leq \tilde{c_{2}} \frac{1}{\sqrt{|x|}} e^{\left(x_{1}-|x|\right) / 2 \mu}+O\left(\frac{1}{\sqrt{|x|}}\right) .
\end{aligned}
$$

Further we calculate

$$
|\mathscr{P} \varphi(x)| \leq \tilde{c_{3}} \max \left(\frac{y_{1}}{|y|}, \frac{y_{2}}{|y|}\right)
$$

with $\tilde{c_{3}}=\frac{1}{\sqrt{2 \pi}}$. Thus in the view of values which are obtained in inequalities (3.2.17), (3.2.18) and (3.2.19), we have

$$
\begin{aligned}
\left|\mathbb{I}_{R}(\varphi)\right| \leq & \|\varphi\|_{\infty}^{2} \int_{|x|=R}\left(\tilde{c}_{1} \frac{1}{\sqrt{|x|}} e^{\left(x_{1}-|x|\right) / 2 \mu}+O\left(\frac{1}{\sqrt{|x|}}\right)\right) \\
& \times\left[\left(\tilde{c}_{3} \max \left(\frac{x_{1}}{|x|}, \frac{x_{2}}{|y|}\right)\right)+\frac{\nu_{1}}{2}\left(\tilde{c}_{2} \frac{1}{\sqrt{|x|}} e^{\left(x_{1}-|x|\right) / 2 \mu}+O\left(\frac{1}{\sqrt{|x|}}\right)\right)\right. \\
& \left.+\left(\tilde{c}_{2} \frac{1}{\sqrt{|x|}} e^{\left(x_{1}-|x|\right) / 2 \mu}+O\left(\frac{1}{\sqrt{|x|}}\right)\right)\right] d s(x) \\
\leq & \tilde{c}\|\varphi\|_{\infty}^{2} \int_{|x|=R}\left(\frac{1}{|x|} e^{\left(x_{1}-|x|\right) / \mu}+O\left(\frac{1}{\sqrt{|x|}}\right)\right) d s(x) \\
\leq & \tilde{c}\|\varphi\|_{\infty}^{2} \int_{|x|=R} \frac{1}{|x|} e^{\left(x_{1}-|x|\right) / \mu} d s(x)+O\left(\frac{1}{\sqrt{R}}\right) d s(x)
\end{aligned}
$$

Now using the parametric representation for the boundary $|x|=R$, i.e.,

$$
x(t)=(R \cos t, R \sin t) \quad t \in[-\pi, \pi],
$$

and with the aid of

$$
\cos t-1=-2 \sin ^{2} t / 2 \leq-2 t^{2} / \pi,
$$


we further estimate the integral in above equation such that

$$
\begin{aligned}
\int_{|x|=R} \frac{1}{|x|} e^{\left(x_{1}-|x|\right) / \mu} d s(x) & =\int_{0}^{2 \pi} e^{\frac{R}{\mu}(\cos t-1)} d t=\int_{-\pi}^{\pi} e^{\frac{R}{\mu}(\cos t-1)} d t \\
& =\int_{-\pi}^{\pi} e^{\frac{-2 R}{\mu} \sin ^{2} \frac{t}{2}} d t \leq \int_{-\pi}^{\pi} e^{\frac{-4 R}{\pi \mu} t^{2}} d t \\
& \leq \frac{\pi \mu}{4 R} \int_{-\pi}^{\pi} e^{-\tau^{2}} d \tau \leq \frac{\pi \mu}{4 R} \int_{-\infty}^{\infty} e^{-\tau^{2}} d \tau \\
& \leq \frac{\mu \sqrt{\pi}}{4 R}
\end{aligned}
$$

Thus with this straight forward calculation, inequality (3.2.20) reduces to

$$
\left|\mathbb{I}_{R}(\varphi)\right| \leq \tilde{C}\|\varphi\|_{\infty}^{2} \frac{1}{R}+O\left(\frac{1}{\sqrt{R}}\right)
$$

Therefore $\mathbb{I}_{R} \rightarrow 0$ as $R \rightarrow 0$ and equation (3.2.14) takes the following form

$$
\mu \int_{D}|\nabla \mathscr{S} \varphi|^{2} d x+\mu \int_{\mathbb{R}^{2} \backslash D}|\nabla \mathscr{S} \varphi|^{2} d x=\langle\varphi, \mathscr{S} \varphi\rangle
$$

Here $\varphi \in C^{0, \alpha}(\partial D)$ and the duality bracket is defined as

$$
\langle\varphi, \mathscr{S} \varphi\rangle:=\int_{\partial D} \varphi \cdot \mathscr{S} \psi d s
$$

on $W^{1 / 2}(\partial D)$. Now in the next step we check whether the density $\varphi$ which belongs to $W^{-1 / 2}(\partial D)$ satisfied the equation $(3.2 .22)$ or not. Since for each $\varphi \in W^{-1 / 2}(\partial D)$ there exists a sequence $\phi_{n} \in C^{0, \alpha}(\partial D)$ such that $\varphi_{n} \rightarrow \varphi$ when $n \rightarrow \infty$ with respect to the norm $W^{-1 / 2}(\partial D)$. Thus from definition 3.2 we can conclude that

$$
\nabla \mathscr{S} \varphi_{n} \rightarrow \nabla \mathscr{S} \varphi, \quad n \rightarrow \infty
$$

with uniform convergence on compact subsets of $D$ and $\mathbb{R}^{2} \backslash \bar{D}$. From theorem 3.4 the operator $\mathscr{S}: W^{-1 / 2}(\partial D) \rightarrow W^{1 / 2}(\partial D)$ is bounded and equation (3.2.22) tells us that $\nabla \mathscr{S} \varphi_{n}$ is a Cauchy sequence in $L^{2}(D)$ and $L^{2}\left(\mathbb{R}^{2} \backslash \bar{D}\right)$ and it is convergent in both $L^{2}(D)$ and $L^{2}\left(\mathbb{R}^{2} \backslash \bar{D}\right)$. From the locally uniform convergence, given in equation(3.2.24), we conclude that

$$
\nabla \mathscr{S} \varphi_{n} \rightarrow \nabla \mathscr{S} \varphi, \quad n \rightarrow \infty
$$

both in $L^{2}(D)$ and $L^{2}\left(\mathbb{R}^{2} \backslash \bar{D}\right)$. Writing equation (3.2.22) in the form

$$
\mu \int_{D}\left|\nabla \mathscr{S} \varphi_{n}\right|^{2} d x+\mu \int_{\mathbb{R}^{2} \backslash D}\left|\nabla \mathscr{S} \varphi_{n}\right|^{2} d x=\langle\varphi, \mathscr{S} \varphi\rangle
$$


and passing the limit $n \rightarrow \infty$ we have

$$
\mu \int_{D}|\nabla \mathscr{S} \varphi|^{2} d x+\mu \int_{\mathbb{R}^{2} \backslash D}|\nabla \mathscr{S} \phi|^{2} d x=\langle\varphi, \mathscr{S} \varphi\rangle
$$

for $\varphi \in W^{-1 / 2}(\partial D)$. Since from Corollary 8.18 in [22] we know that the norm on $W^{1}(B)$, where $B$ is a bounded domain, is $\|u\|_{L^{2}(\partial D)}^{2}+\|\nabla u\|_{L^{2}(D)}^{2}$. Therefore from equation (3.2.26) and Theorem 3.4, we have the boundedness of the operator $\mathscr{S}$ in the domain $B$.

Now we estimate the boundedness of the pressure term $\mathscr{P}$. By definition 3.2 the gradient of $\Phi$, which is the solution of Laplace equation, involves in the operator $\mathscr{P}$. We could enforce Theorem 8.24 from [22] to obtain the boundedness on the operator $\mathscr{P}$.

Theorem 3.6. For $\varphi \in W^{-1 / 2}(\partial D)$ the pair $(u, p)=(\mathscr{S} \varphi, \mathscr{P} \varphi)$ is a weak solution of the Dirichlet problem (3.1.4)-(3.1.6) with boundary condition $u=\mathscr{S} \varphi$ on $\partial D$ and $u_{\infty}=0$.

Proof. The proof of the above theorem is straightforward. Since the pair $(\mathscr{S} \varphi, \mathscr{P} \varphi)$ is the classical solution of the exterior Oseen equation. Using partial integration with the help of Gauss divergence theorem, the equations (3.1.4) and (3.1.6) are satisfied.

Theorem 3.7. The Null space of the operator $\mathscr{S}: W^{-1 / 2}\left(\partial D, \mathbb{R}^{2}\right) \rightarrow W^{1 / 2}\left(\partial D, \mathbb{R}^{2}\right)$ is given by $N(\mathscr{S})=\operatorname{span}\{\nu\}$.

Proof. The proof is given in [23]. However a similar proof is given in Theorem 3.18.

Since the null space of the operator $\mathscr{S}$ is non-trivial so with this operator it is not possible that we could have a unique solution of the Oseen equation. To deal with this issue we need to modify our operator $\mathscr{S}$. By straightforward differentiation we readily see that the pair $\left(\operatorname{grad} \Phi, \partial_{1} \Phi\right)$ is a solution of the Oseen equation. Also from Theorem 3.6, we know that the pair $(\mathscr{S} \varphi, \mathscr{P} \varphi)$ is the solution of Oseen equation provided $u=\mathscr{S} \varphi$ on $\partial D$ and $u_{\infty}=0$. Linear combination of these solutions are solution again.

Lemma 3.8. The operator $U: W^{-1 / 2}\left(\partial D, \mathbb{R}^{2}\right) \rightarrow W^{1 / 2}\left(\partial D, \mathbb{R}^{2}\right)$ defined by

$$
U \varphi=\mathscr{S} \varphi+\langle\varphi, v\rangle \operatorname{grad} \Phi
$$

with $\langle\varphi, \nu\rangle=\int_{\partial D} \varphi \nu d s$ is bijective operator and has a bounded inverse. 
Proof. Following [30], we first prove the injectivity of the operator $U$. Assume that $\langle\nu, U \varphi\rangle=0$ for all $\varphi \in W^{-1 / 2}\left(\partial D, \mathbb{R}^{2}\right)$. Taking the inner product of the operator (3.2.27) with the normal vector $\nu$, we obtain

$$
0=\langle\nu, U \varphi\rangle=\langle\nu, \mathscr{S} \varphi\rangle+\langle\varphi, v\rangle\langle\nu, \operatorname{grad} \Phi\rangle .
$$

Following Theorem 3.5 and because of the divergence theorem we can conclude that $\langle\nu, \mathscr{S} \varphi\rangle=0$, since $\operatorname{div} \mathscr{S} \varphi=0$ in $D$. Moreover, since the origin contained in $D$, from Example 6.16 in [22], we have $\langle\nu, \operatorname{grad} \Phi\rangle=-1$. Together these results with our assumption $U \varphi=0$ the equation (3.2.28) reduces to $\langle\varphi, v\rangle=0$. Also from Theorem 3.7 we know that $\mathscr{S} \varphi=0$ implies that $\varphi \in \operatorname{span}\{\nu\}$. The latter together with $\langle\varphi, v\rangle=0$ gives us our final result that $\varphi=0$. This proves the injectivity of operator $U$.

To prove the rest of the theorem we use this technique. The diffrence $A:=U-\mathscr{S}$ is bounded and has finite dimensional range. Therefore the operator $A$ is a compact operator. The principle part of the equation (3.2.6) is

$$
\left(\mathscr{S}_{0} \psi\right)=\int_{0}^{2 \pi} \ln \left(\frac{4}{e} \sin ^{2} \frac{t-\tau}{2}\right) \psi(\tau) d \tau, \quad t \in[0,2 \pi] .
$$

In the view of Theorem 8.22 of [22], the bounded operator

$$
\mathscr{S}_{0}: W^{r}\left(\partial D, \mathbb{R}^{2}\right) \rightarrow W^{r+1}\left(\partial D, \mathbb{R}^{2}\right)
$$

has a bounded inverse. Also from Theorem 13.20 in [22], we can conclude that $\mathbb{S}-\mathscr{S}_{0}$ is bounded from $W^{r}\left(\partial D, \mathbb{R}^{2}\right)$ into $W^{r+2}\left(\partial D, \mathbb{R}^{2}\right)$ of all $r \in \mathbb{R}$. Thus $\mathbb{S}-\mathscr{S}_{0}$ is compact from $W^{r}\left(\partial D, \mathbb{R}^{2}\right) \rightarrow W^{r+1}\left(\partial D, \mathbb{R}^{2}\right)$ due to the embedding theory of Sobolev spaces. The operator $\mathrm{U}$ is the sum of an invertible operator and a compact operator. We have shown that it is injective and thus, by Riesz theory, the proof is finished.

Theorem 3.9. Given a vector field $f \in W^{\frac{1}{2}}\left(\partial D, \mathbb{R}^{2}\right)$, and a constant vector $u_{\infty}$, the integral equation

$$
U \varphi=f-u_{\infty}
$$

has a unique solution $\varphi \in W^{-\frac{1}{2}}\left(\partial D, \mathbb{R}^{2}\right)$. Then, the pair

$$
\left(\mathscr{S} \varphi+\langle\varphi, v\rangle \operatorname{grad} \Phi+u_{\infty}, \mathscr{P} \varphi-\langle\varphi, v\rangle \partial_{1} \Phi\right)
$$

is a weak solution to the Dirichlet problem (3.1.4)-(3.1.6).

Proof. A proof is worked out in Theorem 2.5 [23].

Usually Oseen problem are solved in exterior domains due to the physical interest in flows. To study the convergence of the point source method for flow reconstruction we need also the wellposedness the interior Oseen problem. It can be shown with similar tools as the exterior problems. Since the proof is not worked out in literature, here we present a concise version for the convenience of the reader. 
Lemma 3.10. For a bounded domain $D \subset \mathbb{R}^{2}$, we introduce a space $\mathcal{X}(D)$ defined by

$$
\mathcal{X}(D):=\left\{\varphi \in C_{0}^{\infty}(D): \nabla \cdot \varphi=0 \text { in } D\right\},
$$

with the scalar product

$$
[\psi, \varphi]:=\int_{D} \nabla \psi: \nabla \varphi d x .
$$

The space $\mathcal{X}(D)$ is a pre-Hilbert space.

Definition 3.11. The space $\widetilde{\mathcal{X}}$ which is defined as the completion of $\mathcal{X}$ with respect to $\|.\| \mathcal{X}$, where

$$
\|\varphi\|_{\mathcal{X}}=[\varphi, \varphi]^{1 / 2}=\left[\int_{D} \nabla \varphi: \nabla \varphi d x\right]^{1 / 2}
$$

Theorem 3.12. Let $D \subseteq \mathbb{R}^{2}, n \geq 2$, be bounded and locally Lipschitzian and assume that $\mu$ is sufficiently large. For any $f \in W^{1 / 2,2}(\partial D)$ with

$$
\int_{\partial D} \nu \cdot f d s=0
$$

there exists one and only one weak solution u to the Oseen problem (3.1.1) in the domain $D$ and satisfy the condition (3.1.2) on boundary. The solution depends continuously on the boundary data in the sense that there is a constant $c$ such that

$$
\|u\|_{W^{1,2}(D)} \leq c\|f\|_{W^{1 / 2,2}(\partial D)} .
$$

Proof. First we remark that under the condition (3.2.33) by Theorem II 3.3 in [16] there exists a solenoidal extension $V \in W^{1,2}(D)$ of $f$, such that we have the estimate

$$
\|V\|_{1,2} \leq c\|f\|_{1 / 2,2(\partial D)}
$$

with some constant $c$. We search the generalized solution $u$ of the Oseen problem in the form

$$
u=w+V,
$$

with $w \in W_{0}^{1,2}(D)$. For $\psi \in \widetilde{\mathcal{X}}(D)$ we remark that we have $\operatorname{div} \psi=0$ and $\left.\psi\right|_{\partial D}=0$, which leads to

$$
\langle\psi, \operatorname{grad} p\rangle=\langle\operatorname{div} \psi, p\rangle=0 .
$$

We study a solution $u$ to (3.1.1), multiply (3.1.1) by $\psi$ and employ partial integration to derive

$$
\mu[u, \psi]-\left\langle u, \partial_{1} \psi\right\rangle=0 .
$$

Plugging (3.2.36) into (3.2.38), we obtain,

$$
\mu[(w+V), \psi]-\left\langle w+V, \partial_{1} \psi\right\rangle=0 .
$$


This leads us to

$$
\mu[w, \psi]-\left\langle w, \partial_{1} \psi\right\rangle=-\mu[V, \psi]+\left\langle V, \partial_{1} \psi\right\rangle,
$$

for all $\psi \in \tilde{\mathcal{X}}(D)$. Using the definition of the bilinear form (3.1.7) for the interior domain $\Omega=D$, the above equation takes the form

$$
a(w, \psi)=-\mu[V, \psi]+\left\langle V, \partial_{1} \psi\right\rangle .
$$

The right hand side of (3.2.40) is a bounded linear functional in $\psi \in \mathcal{X}$. Using the Cauchy Schwarz inequality on both terms of $a(\cdot, \cdot)$ and the Poincaré inequality in the second step we estimate

$$
\begin{aligned}
|a(w, \psi)| & \leq \mu[w, w]^{1 / 2}[\psi, \psi]^{1 / 2}+\langle w, w\rangle^{1 / 2}[\psi, \psi]^{1 / 2} \\
& \leq(\mu+1)\|w\|_{W_{0}^{1,2}(D)}\|\psi\|_{\mathcal{X}(D)} \\
& \leq C(\mu+1)\|w\|_{\mathcal{X}(D)}\|\psi\|_{\mathcal{X}(D)}
\end{aligned}
$$

with some constant $C$, such that $a$ is a bounded sesquilinear form on $\mathcal{X}(D)$. To show coercivity of the sesquilinear form we note

$$
a(w, w)=\mu\|w\|_{\mathcal{X}(D)}^{2}-\left\langle w, \partial_{1} w\right\rangle .
$$

Using the Cauchy Schwarz inequality and the Poincaré inequality on the second term of above equation we estimate

$$
\begin{aligned}
a(w, w) & \geq \mu\|w\|_{\mathcal{X}(D)}^{2}-\langle w, w\rangle^{1 / 2}[w, w]^{1 / 2} \\
& \geq \mu\|w\|_{\mathcal{X}(D)}^{2}-\|w\|_{W_{0}^{1,2}(D)}\|w\|_{\mathcal{X}(D)} \\
& \geq(\mu-C)\|w\|_{\mathcal{X}(D)}^{2}
\end{aligned}
$$

with the Poincaré constant $C$. For $\mu>C$ the sesquilinear form is coercive and by the Lax-Milgram Theorem [12], we can conclude that $w$ is the unique solution of the week formulation of Oseen equation.

Now we have to prove that the solution $w$ is continuously depending on the boundary data. From Lax-Milgram theorem we can conclude that the solutions depends continuously on the boundary data. However we can also get this result with explicit estimates as follows. Replacing $\psi$ with $w$ in equation(3.2.39) we have

$$
\mu[w, w]=\langle w, \partial w\rangle+\langle V, \partial w\rangle-\mu[V, w] .
$$

Via Cauchy Schwarz we estimate

$$
\mu\|w\|_{\mathcal{X}(D)}^{2} \leq\|w\|_{L^{2}(D)}\|w\|_{\mathcal{X}(D)}+\|V\|_{L^{2}(D)}\|w\|_{\mathcal{X}(D)}+\mu\|w\|_{\mathcal{X}(D)}\|\nabla V\|_{L^{2}(D)} .
$$


By the Poincaré inequality we have

$$
\mu\|w\|_{\mathcal{X}(D)}^{2} \leq C\|w\|_{\mathcal{X}(D)}^{2}+\|V\|_{L^{2}(D)}\|w\|_{\mathcal{X}(D)}+\mu\|w\|_{\mathcal{X}(D)}\|\nabla V\|_{L^{2}(D)}
$$

with some constant $C$. Dividing by $\|w\|_{\mathcal{X}(D)}$ with standard estimates we derive

$$
(\mu-C)\|w\|_{\mathcal{X}(D)} \leq(\mu+1)\|\nabla V\|_{W^{1,2}(D)} .
$$

With the estimate (3.2.35) we obtain

$$
(\mu-C)\|w\|_{\mathcal{X}(D)} \leq(\mu+1)\|f\|_{W^{1 / 2,2}(\partial D)} .
$$

Finally, using the Poincaré inequality once again, we can write the above equation as

$$
\frac{(\mu-C)}{C}\|w\|_{W^{1,2}(D)} \leq(\mu+1)\|f\|_{W^{1 / 2,2}(\partial D)} .
$$

So for $\mu>C$ the inequality (3.2.34) is satisfied and the proof is complete.

We first need some preparations before we continue with developing some further integral equation tools for the Oseen problem. We will study the adjoint single layer potential operators. Two operators $A: X \rightarrow Y$ and $A^{*}: Y \rightarrow X$ are said to be adjoint provided that the following equation holds,

$$
\langle A \varphi, \psi\rangle=\left\langle\varphi, A^{*} \psi\right\rangle,
$$

for all $\varphi \in X$ and $\psi \in Y$. Moreover from Theorem 4.6 of [22] we know that for the calculation of the adjoint we need to exchange the role of kernel variables inside the operator, i.e., for an integral operator

$$
(A \varphi)(x)=\int_{\Omega} K(x, y) \varphi(y) d y \quad x \in \Omega
$$

its adjoint is

$$
\left(A^{*} \psi\right)(y)=\int_{\Omega} K(x, y) \psi(x) d x \quad y \in \Omega .
$$

With this brief review of adjoint operators we are going to characterize the adjoint of the single layer potential operators for the Oseen equation. In the formulation of the adjoint Oseen equation we should keep in mind that the fundamental solution $E$ is a tensor and it is symmetric in nature.

Theorem 3.13. Let $\partial D$ be of class $C^{1}$. Assume that $\psi$ be the vector field and $\rho$ be the scalar field on $\partial D$ then in the view of above discussion the adjoint operators of the single layer potential operators $\mathscr{S}$ and $\mathscr{P}$ are

$$
\begin{aligned}
\left(\mathscr{S}^{*} \psi\right)(y) & =\int_{\partial D} E(x-y) \psi(x) d s(x) \\
\left(\mathscr{P}^{*} \rho\right)(y) & =-\int_{\partial D} e(x-y) \rho(x) d s(x)
\end{aligned}
$$


for $y \in \mathbb{R}^{2} \backslash \partial D$, and theses operators must satisfy the following equations

$$
\begin{aligned}
\langle\mathscr{S} \varphi, \psi\rangle & =\left\langle\varphi, \mathscr{S}^{*} \psi\right\rangle, \\
\langle\mathscr{P} \varphi, \rho\rangle & =\left\langle\varphi, \mathscr{P}^{*} \rho\right\rangle .
\end{aligned}
$$

From above Theorem 3.13 it is clear that the operator $\mathscr{S}^{*}$ is for the velocity field and $\mathscr{P}^{*}$ is for the corresponding pressure field. The adjoint of the velocity operator $\mathscr{S}$ maps from vector field to a vector field but the adjoint of the pressure operator $\mathscr{P}$ maps from a scalar field to a vector field which is not acceptable from physical point of view. To address this issue we need to define another operator for the pressure field.

Definition 3.14. Let $\psi=\left(\psi_{1}, \psi_{2}\right)$ be a vector field on $\partial D$ of class $C^{1}$ then we can define the vectorial version of equation (3.2.44) with the operator $\tilde{\mathscr{P}}^{*}$ such that

$$
\left(\tilde{\mathscr{P}}^{*} \psi\right)(y):=-\int_{\partial D} e(x-y) \cdot \psi(x) d s(x) .
$$

We can see that the operator $\tilde{\mathscr{P}}^{*}$ is related to the adjoint of the single layer potential operator $\mathscr{P}$ of Oseen equation, i.e., $\mathscr{P}^{*}$, such that

$$
\begin{aligned}
\left(\tilde{\mathscr{P}}^{*} \psi\right)(y) & =-\int_{\partial D} e(x-y) \cdot \psi(x) d s(x) \\
& =-\int_{\partial D}\left(e_{1}(x-y) \psi_{1}(x)+e_{2}(x-y) \psi_{2}(x)\right) d s(x) \\
& =-\left[\int_{\partial D} e(x-y) \psi_{1}(x) d s(x)\right]_{1}-\left[\int_{\partial D} e(x-y) \psi_{2}(x) d s(x)\right]_{2} \\
& =\left[\mathscr{P}^{*}\left(\psi_{1}\right)\right]_{1}+\left[\mathscr{P}^{*}\left(\psi_{2}\right)\right]_{2} .
\end{aligned}
$$

Lemma 3.15. For an integrable density $\psi \in \partial D$, the pair of adjoint single layer potential operators $\left(\mathscr{S}^{*} \psi, \tilde{\mathscr{P}}^{*} \psi\right)$ is the solution of adjoint Oseen equation (3.1.10).

Proof. By straightforward differentiation we readily see that for $i=1,2$,

$$
\tilde{u}_{i}(y):=E_{i}(x-y), \quad \tilde{p}_{i}(y):=-e_{i}(x-y)
$$

is the solution of adjoint Oseen equation (3.1.10) for fixed $x \in \partial D$. Linear combinations of these solutions are solutions again. Thus for $y \in \mathbb{R}^{2}$ we define

$$
\begin{aligned}
\tilde{u}(y) & :=\int_{\partial D}\left(E_{1}(x-y) \psi_{1}(x)+E_{2}(x-y) \psi_{2}(x)\right) d s(x) \\
& =\int_{\partial D} E(x-y) \psi(x) d s(x) \\
& =\left(\mathscr{S}^{*} \psi\right)(y),
\end{aligned}
$$




$$
\begin{aligned}
\tilde{p}(y) & :=-\int_{\partial D}\left(e_{1}(x-y) \psi_{1}(x)+e_{2}(x-y) \psi_{2}(x)\right) d s(x) \\
& =-\left[\int_{\partial D} e(x-y) \psi_{1}(x) d s(x)\right]_{1}-\left[\int_{\partial D} e(x-y) \psi_{2}(x) d s(x)\right]_{2} \\
& =-\left(\mathscr{P}^{*} \psi_{1}\right)_{1}(y)-\left(\mathscr{P}^{*} \psi_{2}\right)_{2}(y) \\
& =\left(\tilde{\mathscr{P}}^{*} \psi\right)(y) .
\end{aligned}
$$

Then, $(\tilde{u}, \tilde{p})$ or $\left(\mathscr{S}^{*} \psi, \tilde{\mathscr{P}}^{*} \psi\right)$, respectively, is a solution of adjoint Oseen equation (3.1.10) in $D$ and $\mathbb{R}^{2} \backslash \bar{D}$.

Lemma 3.16. Let $\partial D$ be of class $C^{1}$. Let $\nu$ be the outward unit normal on the obstacle $D$. Then for the adjoint single layer potential operators $\mathscr{S}^{*} \psi$ and $\tilde{\mathscr{P}}^{*} \psi$, we have

$$
\begin{aligned}
\lim _{h \rightarrow+0}\left(\mathscr{S}^{*} \psi\right)(y \pm h \nu(y))= & \int_{\partial D} E(x-y) \psi(x) d s(x), \\
\lim _{h \rightarrow+0}\left(\tilde{\mathscr{P}}^{*} \psi\right)(y \pm h \nu(y))= & -\int_{\partial D} e(x-y) \cdot \psi(x) d s(x) \\
& \pm \frac{1}{2} \nu(y) \cdot \psi(y), \\
\lim _{h \rightarrow+0} \frac{\partial\left(\mathscr{S}^{*} \psi\right)}{\partial \nu(y)}(y \pm h \nu(y))= & \int_{\partial D} \frac{\partial E(x-y)}{\partial \nu(y)} \psi(x) d s(x) \\
& \mp \frac{1}{2 \mu}[\psi(y)-\psi(y) \cdot \nu(y) \nu(y)],
\end{aligned}
$$

for $y \in \partial D$.

Proof. Since the jump relations for the single layer potential operators $\mathscr{S}$ and $\mathscr{P}$ are obtained (see Theorem 3.3) using the fact that fundamental solutions of Oseen and Stokes equations have the same singular behavior at origin. As the fundamental solution of Stokes equation has the following property

$$
E_{0}(z)=E_{0}(-z) \quad e(z)=-e(-z)
$$

for $z \in \mathbb{R}^{2}$. Using this useful property and the jump relations defined in Theorem 3.3 we can develop the jump relations for the adjoint operators $\mathscr{S}^{*}$ and $\tilde{\mathscr{P}}^{*}$. The continuity of $\mathscr{S}^{*}$ is obvious, i.e.

$$
\lim _{h \rightarrow+0}\left(\mathscr{S}^{*} \psi\right)(y \pm h \nu(y))=\int_{\partial D} E(x-y) \psi(x) d s(x) .
$$

Further, for $j=1,2$ with the help of $(3.2 .3)$ for $\varphi=(\rho, 0)^{T}$ or $\varphi=(0, \rho)^{T}$ we 
calculate

$$
\begin{aligned}
-\int_{\partial D} e_{j}(x-y \mp h \nu(y)) \rho(x) d s(x) & =\int_{\partial D} e_{j}(y \pm h \nu(y)-x) \rho(x) d s(x) \\
& \stackrel{h \rightarrow 0}{\rightarrow} \int_{\partial D} e_{j}(y-x) \rho(x) d s(x) \pm \frac{1}{2} \nu_{j}(y) \rho(y) \\
& =\int_{\partial D} e_{j}(x-y) \rho(x) d s(x) \pm \frac{1}{2} \nu_{j}(y) \rho(y) .
\end{aligned}
$$

We obtain

$$
\lim _{h \rightarrow+0}\left(\mathscr{P}^{*} \rho\right)(y \pm h \nu(y))=-\int_{\partial D} e(x-y) \rho(x) d s(x) \pm \frac{1}{2} \nu(y) \rho(y) .
$$

The calculation of the jump relation for the adjoint operator defined $\tilde{\mathscr{P}}^{*}$ applied to a vector field $\psi$ defined in (3.2.47) is:

$$
\begin{aligned}
\lim _{h \rightarrow+0}\left(\tilde{\mathscr{P}}^{*} \psi\right)(y \pm h \nu(y))= & {\left[\lim _{h \rightarrow+0} \mathscr{P}^{*} \psi_{1}(y \pm h \nu(y))\right]_{1} } \\
& +\left[\lim _{h \rightarrow+0} \mathscr{P}^{*} \psi_{2}(y \pm h \nu(y))\right]_{2} \\
= & {\left[-\int_{\partial D} e(x-y) \psi_{1}(x) d s(x) \pm \frac{1}{2} \nu(y) \psi_{1}(y)\right]_{1} } \\
& +\left[-\int_{\partial D} e(x-y) \psi_{2}(x) d s(x) \pm \frac{1}{2} \nu(y) \psi_{2}(y)\right]_{2} \\
= & -\int_{\partial D}\left[e_{1}(x-y) \psi_{1}(x)+e_{2}(x-y) \psi_{2}(x)\right] d s(x) \\
& \pm \frac{1}{2}\left[\nu_{1}(y) \psi_{1}(y)+\nu_{2}(y) \psi_{2}(y)\right] \\
= & -\int_{\partial D} e(x-y) \cdot \psi(x) d s(x) \pm \frac{1}{2} \nu(y) \cdot \psi(y) .
\end{aligned}
$$

A similar calculation for $\mathscr{S}^{*}$ shows

$$
\begin{aligned}
\lim _{h \rightarrow+0} \frac{\mathscr{S}^{*} \psi}{\partial \nu}(y \pm h \nu(y))= & \int_{\partial D} \frac{\partial E(x-y)}{\partial \nu(y)} \psi(x) d s(x) \\
& \mp \frac{1}{2 \mu}[\varphi(y)-(\varphi(y) \cdot \nu(y)) \nu(y)]
\end{aligned}
$$

for $y \in \partial D$.

Lemma 3.17. Let $D$ be some domain with boundary $\partial D$ of class $C^{1}$. Given a vector field $\psi \in L^{2}(\partial D)$, then

$$
\mu \int_{D}\left|\nabla \mathscr{S}^{*} \psi\right|^{2} d y+\mu \int_{\mathbb{R} \backslash \bar{D}}\left|\nabla \mathscr{S}^{*} \psi\right|^{2} d y=\int_{\partial D} \psi \cdot \mathscr{S}^{*} \psi d s .
$$


Proof. From Lemma 3.15 we note that $(\tilde{u}, \tilde{p})=\left(\mathscr{S}^{*} \psi, \tilde{P}^{*} \psi\right)$ satisfies the adjoint Oseen equation in $D$ and in $\mathbb{R}^{2} \backslash \bar{D}$. Since adjoint Oseen equation is an Oseen equation with different signs. To prove equation (3.2.55) we follow the same arguments which we used before for the Oseen equation in Theorem 3.5. However we repeat some of the arguments due to the change in sign for the convenience of the reader. Working on equation (3.1.10) and with the help of Gauss divergence theorem we derive

$$
\mu \int_{D}|\nabla \tilde{u}|^{2} d y=\int_{\partial D} \tilde{u} \cdot\left(\mu \frac{\partial \tilde{u}}{\partial \nu}+\frac{\nu_{1}}{2} \tilde{u}-\tilde{p} \nu\right) d s .
$$

Now we employ the jump relations (3.2.48), (3.2.49) to calculate

$$
\begin{aligned}
\mu \int_{D}\left|\nabla \mathscr{S}^{*} \psi\right|^{2} d y= & \int_{\partial D} \mathscr{S}^{*} \psi \cdot\left(\mu \frac{\partial \mathscr{S}^{*} \psi}{\partial \nu}+\frac{\nu_{1}}{2} \mathscr{S}^{*} \psi-\left(\tilde{\mathscr{P}}^{*} \psi\right) \nu\right) d s \\
& +\frac{1}{2} \int_{\partial D} \psi \cdot \mathscr{S}^{*} \psi d s
\end{aligned}
$$

In a second step we apply (3.2.56) to $D_{R}:=\mathbb{R}^{2} \backslash \bar{D}$. In this case we have two boundaries i.e., $\partial D$ and $\partial D_{R}$. Thus with the help of the jump relations, equation (3.2.56) takes the form

$$
\begin{aligned}
\mu \int_{D_{R}}\left|\nabla \mathscr{S}^{*} \psi\right|^{2} d y= & \int_{|x|=R} \mathscr{S}^{*} \psi \cdot\left(\mu \frac{\partial \mathscr{S}^{*} \psi}{\partial \nu}+\frac{\nu_{1}}{2} \mathscr{S}^{*} \psi-\left(\tilde{\mathscr{P}}^{*} \psi\right) \nu\right) d s . \\
& -\int_{\partial D} \mathscr{S}^{*} \psi \cdot\left(\mu \frac{\partial \mathscr{S}^{*} \psi}{\partial \nu}+\frac{\nu_{1}}{2} \mathscr{S}^{*} \psi-\left(\tilde{\mathscr{P}}^{*} \psi\right) \nu\right) d s \\
& +\frac{1}{2} \int_{\partial D} \psi \cdot \mathscr{S}^{*} \psi d s
\end{aligned}
$$

Finally adding equation (3.2.57) and equation (3.2.58) we have

$$
\mu \int_{D}\left|\nabla \mathscr{S}^{*} \psi\right|^{2} d y+\mu \int_{D_{R}}\left|\nabla \mathscr{S}^{*} \psi\right|^{2} d y=\mathbb{I}_{R}+\int_{\partial D} \psi \cdot \mathscr{S}^{*} \psi d s
$$

where

$$
\mathbb{I}_{R}=\int_{|x|=R} \mathscr{S}^{*} \psi \cdot\left(\mu \frac{\partial \mathscr{S}^{*} \psi}{\partial \nu}+\frac{\nu_{1}}{2} \mathscr{S}^{*} \psi-\left(\tilde{\mathscr{P}}^{*} \psi\right) \nu^{T}\right) d s
$$

As in the proof of Theorem 3.5 it is proved that $\mathbb{I}_{R} \rightarrow 0$ as $R \rightarrow \infty$. Thus, taking limit $R \rightarrow \infty$ in equation (3.2.59), we obtain equation (3.2.55).

Theorem 3.18. Let $D \subset \mathbb{R}^{2}$ be a domain with at least $C^{1}$ boundary. Then the null space of the operator $\mathscr{S}^{*}: L^{2}(\partial D) \rightarrow L^{2}(\partial D)$ is given by

$$
N\left(\mathscr{S}^{*}\right)=\operatorname{span}\{\nu\} .
$$


Proof. Assume $\mathscr{S}^{*} \psi=0$ on $\partial D$ for $\psi \in L^{2}(\partial D)$. By equation (3.2.55) we have

$$
\mu \int_{D}\left|\nabla \mathscr{S}^{*} \psi\right|^{2} d x+\mu \int_{\mathbb{R}^{2} \backslash \bar{D}}\left|\nabla \mathscr{S}^{*} \psi\right|^{2} d x=\left\langle\psi, \mathscr{S}^{*} \psi\right\rangle_{L^{2}(\partial D)}
$$

From above equation we have $\mathscr{S}^{*} \psi$ is constant on $\partial D$. Then by the adjoint Oseen equation (3.1.10), we also have that $\tilde{\mathscr{P}}^{*} \psi$ is constant in $D$ and in $\mathbb{R}^{2} \backslash \bar{D}$ and with the help of jump relations (3.2.48), (3.2.49) and (3.2.50), we can conclude that

$$
c=\nu \cdot \varphi, \quad 0=\varphi-(\nu \cdot \varphi) \nu=\varphi-c \nu,
$$

which implies $\psi \in \operatorname{span}\{\nu\}$.

Conversely, we consider $\psi=\nu$. Since, by the adjoint Oseen equation, $\operatorname{div} S^{*} \nu=0$ in $\mathbb{R}^{2} \backslash \bar{D}$ Gauss' theorem yields

$$
\int_{\partial D} \nu \cdot \mathscr{S}^{*} \nu d s=0
$$

From equation (3.2.61) we conclude $\mathscr{S}^{*} \nu$ is a constant in $\mathbb{R}^{2} \backslash \bar{G}$. Since $\left(\mathscr{S}^{*} \nu\right)(x)$ tends to zero for $|x| \rightarrow \infty$ we conclude $\mathscr{S}^{*} \nu=0$ in $\mathbb{R}^{2} \backslash \bar{D}$. This yields $\mathscr{S}^{*} \nu=0$ on $\Lambda$ and completes the proof.

We will now work out an alternative existence proof for the interior problem via integral equations, which also provides continuity statements for later use.

Theorem 3.19. The operator $\mathscr{S}$ is boundedly invertible from

$$
\operatorname{span}\{\nu\}^{\perp}=N(\mathscr{S})^{\perp} \subset W^{-1 / 2}(\partial D)
$$

into

$$
\operatorname{span}\{\nu\}^{\perp}=N\left(\mathscr{S}^{*}\right)^{\perp} \subset W^{1 / 2}(\partial D) .
$$

Proof. We remark that $\mathscr{S}=\mathscr{S}_{0}+\left(\mathscr{S}-\mathscr{S}_{0}\right)$ with a compact operator $\mathscr{S}-\mathscr{S}_{0}$ : $W^{-1 / 2} \rightarrow W^{1 / 2}$ and the principal part which in parametrized form is given in (3.2.29). The operator $\mathscr{S}_{0}$ is boundedly invertible from $W^{-1 / 2}(\partial D)$ into $W^{1 / 2}(\partial D)$, compare Theorem 8.22 of [22]. For $\mathscr{S}$ we remark that according to Lemma 3.18 we have

$$
\overline{R(\mathscr{S})}=N\left(\mathscr{S}^{*}\right)^{\perp}=\operatorname{span}\{\nu\}^{\perp}=\left\{\varphi \in W^{1 / 2}(\partial D):\langle\nu, \varphi\rangle=0\right\} .
$$

We define the auxiliary operator

$$
(\tilde{U} \varphi)(x):=(\mathscr{S} \varphi)(x)+\langle\varphi, \nu\rangle \nu(x), \quad x \in \partial D .
$$

Clearly, the second term in $\tilde{U}$ has finite dimensional image and is thus compact, and thus $\tilde{U}$ is sum of a boundedly invertible and a compact operator. If we can show injectivity of $\tilde{U}$ from $W^{-1 / 2}(\partial D)$ into $W^{1 / 2}(\partial D)$, by the Riesz-Fredholm theory 
we obtain invertibility and the boundedness of the inverse. To show injectivity we assume that $\varphi$ with $\tilde{U} \varphi=0$. Then

$$
0=\langle\nu, \tilde{U} \varphi\rangle=\underbrace{\langle\nu, \mathscr{S} \varphi\rangle}_{=\left\langle\mathscr{S}^{*} \nu, \varphi\right\rangle=0}+\langle\varphi, \nu\rangle \underbrace{\langle\nu, \nu\rangle}_{\neq 0}
$$

which yields $\langle\varphi, \nu\rangle=0$. Now $0=\tilde{U} \varphi=\mathscr{S} \varphi$ leads to $\varphi=\beta \nu$ with some $\beta \in \mathbb{R}$, since $N(\mathscr{S})=\operatorname{span}\{\nu\}$. Finally, from (3.2.63) we now obtain $0=\beta\langle\nu, \nu\rangle$, which yields $\beta=0$ and thus $\varphi=0$. Thus, we have shown that $\tilde{U}$ is boundedly invertible.

Let $\varphi$ be a solution of $\tilde{U} \varphi=f$ where $\langle f, \nu\rangle=0$. Then we know that

$$
0=\langle\nu, f\rangle=\langle\nu, \tilde{U} \varphi\rangle=\langle\nu, \mathscr{S} \varphi\rangle+\langle\varphi, \nu\rangle\langle\nu, \nu\rangle=\langle\varphi, \nu\rangle\langle\nu, \nu\rangle .
$$

We conclude that $\langle\varphi, \nu\rangle=0$ and thus $\varphi \in N(\mathscr{S})^{\perp}$ and $\mathscr{S} \varphi=f$. This proves that $\mathscr{S}$ is boundedly invertible as operator $N(\mathscr{S})^{\perp} \rightarrow N\left(\mathscr{S}^{*}\right)^{\perp}$, and the proof is complete.

We can use the above integral equation of the first kind to solve the interior Oseen problem.

Corollary 3.20. (Interior Problem) Let $\varphi \in W^{-1 / 2}(\partial D)$ be a solution to

$$
\mathscr{S} \varphi=f
$$

for $f \in W^{1 / 2}(\partial D)$ with $\langle f, \nu\rangle=0$. Then the pair $(\mathscr{S} \varphi, \mathscr{P} \varphi)$ is a weak solution to the interior Oseen equation in $D$. The solution $(\mathscr{S} \varphi, \mathscr{P} \varphi)$ in $W^{1}(D) \times L^{2}(D)$ depends continuously on $f$.

Proof. Clearly $(\mathscr{S} \varphi, \mathscr{P} \varphi)$ solves the Oseen equation and the boundary values of $\mathscr{S} \varphi$ are given by $f$ according to Theorem 3.19. Continuity is a consequence of the boundedness of the inverse in combination with the boundedness of the potential operators as established in Theorem 3.5.

Corollary 3.21. (Exterior Problem) Let $\varphi \in W^{-1 / 2}(\partial D)$ be a solution to

$$
\mathscr{S} \varphi=f
$$

for $f \in W^{1 / 2}(\partial D)$ with $\langle f, \nu\rangle=0$. Then the pair $(\mathscr{S} \varphi, \mathscr{P} \varphi)$ is a weak solution to the exterior Oseen equation in $\mathbb{R}^{2} \backslash \bar{D}$. The solution $(\mathscr{S} \varphi, \mathscr{P} \varphi)$ in $W_{\text {loc }}^{1}\left(\mathbb{R}^{2} \backslash \bar{D}\right) \times$ $L_{\text {loc }}^{2}\left(\mathbb{R}^{2} \backslash \bar{D}\right)$ depends continuously on $f$.

Proof. With the condition $\langle f, \nu\rangle=0$ this Corollary is an extension of Theorem 3.6. 
As a consequence of the above Corollary 3.20 there are constants $c, c^{\prime}$ such that if we have two solutions $(u, p)$ and $(\tilde{u}, \tilde{p})$ of the interior Oseen problem with

$$
\|u-\tilde{u}\|_{W^{1 / 2}(\partial D)} \leq \epsilon
$$

then

$$
\|u-\tilde{u}\|_{W^{1}(D)} \leq c \epsilon, \quad\|p-\tilde{p}\|_{L^{2}(D)} \leq c^{\prime} \epsilon
$$

Since the adjoint Oseen equation is an Oseen equation with different direction of the flow $u_{0}$, the same estimates apply to solutions of the adjoint Oseen equation. 


\section{Chapter 4}

\section{Field Reconstructions by the Point Source Method}

The point source method is a well established scheme for field reconstruction in acoustics and electromagnetics, see [6, 11, 25, 26, 28, 34-36, 38]. It belongs to the class of decomposition methods in inverse scattering since it solves the non-linear and ill-posed inverse shape reconstruction problem by a decomposition into a linear ill-posed problem and non-linear well-posed problem. Here, our goal is to establish the point source method (PSM) for the reconstruction of fluid flow phenomena.

In this chapter we investigate the point source method for the Oseen flow. The Oseen equation is basically derived from the Navier-Stokes equation which is vectorial in nature. Before this the point source method is applied on the Helmholtz's or Maxwell's equations successfully $[28,35,36]$. However, here we can see the new aspects of point source method. In contrast to acoustics or electromagnetics, the use of the point source method in fluid dynamics leads to a number of challenges in terms of the analysis and the proper setup of the scheme, in particular since the null-spaces of the integral operators under consideration are no longer trivial.

We will base the point source method on an integral equation approach to represent the flow field in the exterior of some object $D$. This approach contains integrals over the unknown inclusion. These terms are removed by using an approximation of the point source by a single layer potential over the measurement surface and a reduction step where the remaining term is expressed in terms of measured data.

Our primary task is to reconstruct the flow field and to locate and reconstruct the shape of unknown obstacles from the given velocity field at infinity. Assume that the obstacle $D \subset \mathbb{R}^{2}$ and a domain $\Omega$ such that $D \subset \Omega$. We want to find an approximating function $\Upsilon(x)$, defined as

$$
\Upsilon(x):=\int_{\Lambda} E(y-x) g(y) d s(y), \quad x \in \Omega,
$$

for the point source $E(x-\cdot)$ with source point $x$ located in the region $\Omega \backslash \bar{D}$, here 
$\Lambda$ is an open subset of the boundary $\partial \Omega$ of the domain $\Omega$. Since the fundamental solution of Oseen equation $E(x-\cdot)$ is a matrix of dimension $2 \times 2$. Therefore the density $g \in\left(L^{2}(\Lambda)\right)^{2 \times 2}$ is tensor of dimension $2 \times 2$.

In a first step we establish the layer potential theory to approximate the point source $E(x-\cdot)$ to approximating function $\Upsilon(\cdot)$. For the inverse problem our obstacle $D$ is unknown so we replace the unknown domain $D$ with a known domain, called approximation domain, $G_{x}$ such that $G \subset \Omega$. The point source method usually works with approximation domains $G$, where $D \subset G$ is a sufficient condition for convergence of the reconstruction of the flow field $u(x)$ in a point $x \notin \bar{G}$.

Furthermore, the approximation of the point source by a single-layer potential contains the ill-posed part of the reconstruction, it is carried out by means of Tikhonov regularization. This then leads to a reconstruction formula

$$
u_{r e c}(x)=\int_{\Lambda} g^{T}(\xi)\left(u_{\text {meas }}-u_{\infty}\right)(\xi) d s(\xi)+u_{\infty}, \quad x \in \mathbb{R}^{2} \backslash \bar{D},
$$

with some density tensor $g \in\left(L^{2}(\Lambda)\right)^{2 \times 2}$ and the measured values $u_{\text {meas }}$ on some surface $\Lambda$.

The plan for this chapter is as follows. In section 4.1 we introduce the methodology for the choice of approximation domain $G$ and presented the approximation of the source point $E(x, \cdot)$ with the single layer potential operator. Then in section 4.3 we construct the back projection formula for the Oseen flow with the help of point source method. In this monograph we did not provide any new results to prove the uniqueness of the inverse problem. However following Kress and Meyer [23], uniqueness for the inverse problem is demonstrated in section 4.2.

\subsection{Approximation with a Single-Layer Potential}

The basic goal of this work is to reconstruct the flow field and the shape of unknown obstacle $D$ due to the Oseen's flow. This means that in the first step we replace the unknown domain $D$ with the known domain $G$. The position of the new domain named as approximation domain $G$ is subject to the source point $x$. Thus for all $x \in \Omega \backslash \bar{D}$ we choose the approximation domain $G_{x}$ such that $\bar{D} \subset G_{x}$ and $x \notin G_{x}$ for approximating the point source $E(x-\cdot)$.

As the setting for the single layer potential operator is different for the Oseen equation as compared to Laplace equation or Helmholtz equation. The integral operators under consideration have non-trivial null-spaces and to prove the approximation properties of the operators we need a careful consideration of these spaces. As another point, the fundamental solution of the Oseen equation is not symmetric or antisymmetric in its arguments, as it is for acoustic problems. The other difference is that Oseen equation has two dependent variables i.e. velocity field and pressure. Therefore the single layer potential operator for Oseen equation is a pair 
of two operators (see definition 3.2), one is for the velocity field and the other one is for the corresponding pressure field. This makes things much more involved, when we carry out the setup and proofs for the approximation properties, in particular we need to investigate the adjoint operators, which solve some adjoint Oseen problem. We will address and resolve the problems and provide a full convergence analysis. Since the problem is tensorial by nature and we need to work with a tensorial fundamental solution. We introduce the following version of the single layer potential operators for the Oseen equation or the adjoint Oseen equation.

Definition 4.1. Let $\Lambda$ and $\partial G$ are the boundaries of the domains $G \subset \mathbb{R}^{2}$ and $\Omega \subset \mathbb{R}^{2}$, respectively. Let $g=\left(g_{1}, g_{2}\right)$ be a matrix of dimension $2 \times 2$ with $g_{i} \in$ $\left(L^{2}(\partial G)\right)^{2 \times 1}$ for $i=1,2$. Then the pair of single layer potential operators

$$
S:\left(L^{2}(\partial G)\right)^{2 \times 2} \rightarrow\left(L^{2}(\Lambda)\right)^{2 \times 2}, \quad P:\left(L^{2}(\partial G)\right)^{2 \times 2} \rightarrow\left(L^{2}(\Lambda)\right)^{1 \times 2},
$$

is defined by

$$
\begin{array}{rlrl}
(S g)(y):= & \int_{\partial G} E(y-\xi) g(\xi) d s(\xi), & y \in \Lambda, \\
(P g)(y):=\int_{\partial G}(\nabla \Phi(y-\xi))^{T} g(\xi) d s(\xi), & y \in \Lambda .
\end{array}
$$

Here $E(x-y)$ and $\nabla \Phi(y-\xi)$ represents fundamental solution of the Oseen equation defined in equation (3.1.32) and (3.1.33).

Furthermore the operators $S$ and $P$ have the adjoint operators $S^{*}$ and $P^{*}$ such that

$$
\langle S g, \tilde{g}\rangle=\left\langle g, S^{*} \tilde{g}\right\rangle \quad\langle P g, \tilde{g}\rangle=\left\langle g, P^{*} \tilde{g}\right\rangle,
$$

for all $g \in L^{2}(\partial G)$ and $\tilde{g} \in L^{2}(\Lambda)$. We can write the operators (4.1.2) and (4.1.3) in the shape of pairs, when applied on a matrix $g$, such that $S g=\left(\mathscr{S} g_{1}, \mathscr{S} g_{2}\right)$ and $P g=\left(\mathscr{P} g_{1}, \mathscr{P} g_{2}\right)$. The operator

$$
\mathscr{S}:\left(L^{2}(\partial G)\right)^{2 \times 1} \rightarrow\left(L^{2}(\Lambda)\right)^{2 \times 1},
$$

is defined as

$$
(\mathscr{S} \psi)(y):=\int_{\partial G} E(y-\xi) \psi(\xi) d s(\xi) \quad y \in \Lambda,
$$

for any integrable density $\psi \in\left(L^{2} \partial G\right)^{2 \times 1}$. Similarly the operator $P$ can be written in the form of a pair $\left(\mathscr{P} g_{1}, \mathscr{P} g_{2}\right)$. Here the operator

$$
\mathscr{P}(y):\left(L^{2}(\partial G)\right)^{2 \times 1} \rightarrow\left(L^{2}(\Lambda)\right)^{1 \times 1},
$$

is defined as

$$
(\mathscr{P} \psi):=\int_{\partial G}(\nabla \Phi(y-\xi))^{T} \psi(\xi) d s(\xi) \quad y \in \Lambda .
$$

The idea of writing operators in the form of a pair can be extended up to their adjoint operators such that $S^{*} \tilde{g}=\left(\mathscr{S}^{*} \tilde{g}_{1}, \mathscr{S}^{*} \tilde{g}_{2}\right)$ and $P^{*} \tilde{g}=\left(\mathscr{P}^{*} \tilde{g}_{1}, \mathscr{P}^{*} \tilde{g}_{2}\right)$. 
Remark: As a remake we should mention that the pair $(\mathscr{S} \psi, \mathscr{P} \psi)$ satisfy the Oseen equation on the boundary $\Lambda$.

Definition 4.2. Let $\partial G$ and $\Lambda$ are defined as in Definition 4.1. Let $g=\left(g_{1}, g_{2}\right)$ is a matrix of dimension $2 \times 2$ with $g_{i} \in\left(L^{2}(\Lambda)\right)^{2 \times 1}$ for $i=1,2$. Then the pair of single layer potential operators for the adjoint Oseen equation is defined by

$$
\begin{array}{lll}
\left(S^{(a d j)} g\right)(\xi) & :=\int_{\Lambda} E(y-\xi) g(y) d s(y), & \xi \in \mathbb{R}^{2} \backslash \Lambda, \\
\left(P^{(a d j)} g\right)(\xi) & :=-\int_{\Lambda}(\nabla \Phi(y-\xi))^{T} g(y) d s(y), & \xi \in \mathbb{R}^{2} \backslash \Lambda .
\end{array}
$$

Since the operators $S^{(a d j)}$ and $P^{(a d j)}$ are applied to the matrix $g$ we can rewrite them as following

$$
\begin{aligned}
& S^{(a d j)} g:=\left(\mathscr{S}^{(a d j)} g_{1}, \mathscr{S}^{(a d j)} g_{2}\right), \\
& P^{(a d j)} g:=\left(\mathscr{P}^{(a d j)} g_{1}, \mathscr{P}^{(a d j)} g_{2}\right) .
\end{aligned}
$$

For any arbitrary density $\varphi \in\left(L^{2}(\Lambda)\right)^{2 \times 1}$, the operator

$$
\mathscr{S}^{(a d j)}:\left(L^{2}(\Lambda)\right)^{2 \times 1} \rightarrow\left(L_{l o c}^{2}\left(\mathbb{R}^{2} \backslash \Lambda\right)\right)^{2 \times 1}
$$

is defined as

$$
\left(\mathscr{S}^{(a d j)} \varphi\right)(\xi)=\int_{\Lambda} E(y-\xi) \varphi(y) d s(y), \quad \xi \in \mathbb{R}^{2} \backslash \Lambda
$$

and the pressure field operator

$$
\mathscr{P}^{(a d j)}:\left(L^{2}(\Lambda)\right)^{2 \times 1} \rightarrow\left(L_{l o c}^{2}\left(\mathbb{R}^{2} \backslash \Lambda\right)\right)^{1 \times 1}
$$

is defined as

$$
\left(\mathscr{P}^{(a d j)} \varphi\right)(\xi)=-\int_{\Lambda} \underbrace{(\nabla \Phi(y-\xi))^{T}}_{1 \times 2} \underbrace{\varphi(y)}_{2 \times 1} d s(y) \quad \xi \in \mathbb{R}^{2} \backslash \Lambda .
$$

These operators coincide with the classical adjoint of the single layer potentials for the Oseen equation (3.1.1) (see Theorem 3.13) .

Lemma 4.3. For an integrable density $\varphi \in\left(L^{2}(\Lambda)\right)^{2 \times 1}$, the pair of single layer potential operators $\left(\mathscr{S}^{(a d j)} \varphi, \mathscr{P}^{(a d j)} \varphi\right)$ defined in equations (4.1.11) and (4.1.13) satisfies the adjoint Oseen equation (3.1.10) in $\mathbb{R}^{2} \backslash \Lambda$.

Proof. We know that the pairs $\left(E_{i}(y-\xi), e_{i}(y-\xi)\right), i=1,2$ are two linearly independent solutions of the Oseen equation (3.1.1) for fixed $y \in \Lambda$. From a straight 
forward calculation we see that $\left(E_{i}(y-\xi),-e_{i}(y-\xi)\right), i=1,2$ are two linearly independent solutions of adjoint Oseen equation (3.1.10) for fixed $y \in \Lambda$. Since linear combinations of these solutions is again a solution, for $\xi \in \mathbb{R}^{2} \backslash \Lambda$ we can define

$$
\begin{aligned}
\tilde{u}(\xi) & :=\int_{\Lambda}\left(E_{1}(y-\xi) \varphi_{1}(y)+E_{2}(y-\xi) \varphi_{2}(y)\right) d s(y) \\
& =\int_{\Lambda} E(y-\xi) \varphi(y) d s(y) \\
& =\left(\mathscr{S}^{(a d j)} \varphi\right)(\xi), \\
\tilde{p}(\xi) & :=-\int_{\Lambda}\left(e_{1}(y-\xi) \varphi_{1}(y)+e_{2}(y-\xi) \varphi_{2}(y)\right) d s(y) \\
& =-\int_{\Lambda} e(y-\xi) \cdot \varphi(y) d s(y) \\
& =\left(\mathscr{P}^{(a d j)} \varphi\right)(\xi),
\end{aligned}
$$

where $\varphi \in\left(L^{2}(\Lambda)\right)^{2 \times 1}$. Thus, $(\tilde{u}, \tilde{p})$ or $\left(\mathscr{S}^{(a d j)} \varphi, \mathscr{P}^{(a d j)} \varphi\right)$, respectively, is a solution of adjoint Oseen equation (3.1.10) in $\mathbb{R}^{2} \backslash \Lambda$.

Remark: If we choose the appropriate domains $G$ and $\Omega$ such that $G \subset \Omega$ then $\partial G$ must belong to the region $\mathbb{R}^{2} \backslash \Lambda$. Then the adjoint of the single layer potential operator (4.1.2) for the Oseen equation is equal to the potential operators $S^{(a d j)}$, i.e.,

$$
S^{(a d j)}=S^{*} .
$$

In future, unless otherwise stated, the operator $S^{(a d j)}$ goes from $\left(L^{2}(\Lambda)\right)^{2 \times 2}$ to $\left(L^{2}(\partial G)\right)^{2 \times 2}$. Similarly the operator $P^{(a d j)}$ goes from $L^{2}(\Lambda)^{2 \times 2}$ to $\left(L^{2}(\partial G)\right)^{1 \times 2}$.

Due to the wellposedness of the Oseen equation and the adjoint Oseen equation in the interior domain (see Remark after the Corollary 3.20) it is sufficient to work out on one operator. If we get the approximation for the one operator then the approximation for the other one is understood in the sense (3.2.66).

Now let us consider the Oseen equation in the domain $\Omega \backslash \bar{G}$. We will show that we can find an approximating function $\Upsilon$ in the form of a single-layer potential operator

$$
\Upsilon(z)=\int_{\Lambda} E(y-z) g(y) d s(y) \quad z \in \Omega
$$

of the corresponding adjoint Oseen equation which approximates the point source $E(x-\cdot)$ on $G$ such that

$$
E(x-\cdot) \approx \Upsilon(\cdot) \quad x \notin G .
$$

The point source method is based on the principle that the single layer potential operator has a dense range in a $L^{2}$ space. Under this principle it has been successfully applied for different problems (See for example [34-36, 38]). However in our 
problem this criterion is not fulfilled. The operator $S^{(a d j)}$ do not have a dense range in $\left(L^{2}(\partial G)\right)^{2 \times 2}$ since the adjoint operator for the Oseen equation is not injective. To deal with this problem we adopted another technique to implement the point source method for the reconstruction of flow field.

Theorem 4.4. Assume that $\Lambda$ is an open subset of the boundary $\partial B$ of a domain $B$ with analytical boundary containing $\partial G$ in its interior. Then, the null space of the operator $\mathscr{S}:\left(L^{2}(\partial G)\right)^{2 \times 1} \rightarrow\left(L^{2}(\Lambda)\right)^{2 \times 1}$ is given by

$$
N(\mathscr{S})=\operatorname{span}\{\nu\} .
$$

Proof. Assume $\mathscr{S}^{*} \psi=0$ on $\Lambda$ for $\psi \in\left(L^{2}(\partial G)\right)^{2 \times 1}$. By using (3.2.22) applied to the approximation domain $G$, we have

$$
\mu \int_{G}|\nabla \mathscr{S} \psi|^{2} d x+\mu \int_{\mathbb{R}^{2} \backslash \bar{G}}|\nabla \mathscr{S} \psi|^{2} d x=\langle\psi, \mathscr{S} \psi\rangle_{\left(L^{2}(\partial G)\right)^{2 \times 1}} .
$$

From $\mathscr{S} \psi=0$ on $\Lambda$, by analyticity of $\mathscr{S} \psi$ on $\partial B$, we obtain $\mathscr{S} \psi \equiv 0$ on $\partial B$. Clearly, the pair $(\mathscr{S} \psi, \mathscr{P} \psi)$ is a weak solution of the Oseen equation in $\mathbb{R}^{2} \backslash \bar{B}$. Uniqueness (Theorem VII.1.2 in [16]) of the exterior Oseen problem implies $\mathscr{S} \psi \equiv 0$ in $\mathbb{R}^{2} \backslash \bar{B}$. Since domain $B$ contains $G$ in its interior, by analytic continuation into $\mathbb{R}^{2} \backslash \bar{G}$ and the continuity of $\mathscr{S} \psi$ on $\partial G$ we obtain $\mathscr{S} \psi=0$ on $\partial G$. Now equation (4.1.17) implies that $\mathscr{S} \psi$ is constant in $G$ and in $\mathbb{R}^{2} \backslash \bar{G}$. Then by the Oseen equation (3.1.1), we also have that $\mathscr{P} \psi$ is constant in $G$ and in $\mathbb{R}^{2} \backslash \bar{G}$ then with the help of jump relations (3.2.3) and (3.2.4) we finally have $\psi \in \operatorname{span}\{\nu\}$.

Conversely, we consider $\psi=\nu$. Since, by the Oseen equation, $\operatorname{div} \mathscr{S} \nu=0$ in $\mathbb{R}^{2} \backslash \bar{G}$ Gauss' theorem yields

$$
\int_{\partial G} \nu \cdot \mathscr{S} \nu d s=0
$$

From equation (4.1.17) we conclude $\mathscr{S} \nu$ is a constant in $\mathbb{R}^{2} \backslash \bar{G}$. Since $(\mathscr{S} \nu)(x)$ tends to zero for $|x| \rightarrow \infty$ we conclude $\mathscr{S} \nu=0$ in $\mathbb{R}^{2} \backslash \bar{G}$. This yields $\mathscr{S} \nu=0$ on $\Lambda$ and completes the proof.

Corollary 4.5. Assume that $\Lambda$ and $\partial G$ satisfied the assumptions of Theorem 4.4. Then the operator $\mathscr{S}^{(a d j)}:\left(L^{2}(\Lambda)\right)^{2 \times 1} \rightarrow\left(L^{2}(\partial G)\right)^{2 \times 1}$ has a non-trivial null space such that

$$
N\left(\mathscr{S}^{(a d j)}\right)=\operatorname{span}\{\nu\}
$$

Proof. A similar proof is done in Theorem 3.18. 
Theorem 4.6. The nullspace of the operator $S$ and the nullspace of the operator $S^{(a d j)}$ are given by

$$
\begin{aligned}
N(S) & =\operatorname{span}\{(\nu, 0),(0, \nu)\}, \\
N\left(S^{(a d j)}\right) & =\operatorname{span}\{(\nu, 0),(0, \nu)\},
\end{aligned}
$$

respectively.

Proof. Since the proof of equations (4.1.19) and (4.1.20) are similar, here we present the proof for the operator $S$. Consider a matrix $g=\left(g_{1}, g_{2}\right)$ of size $2 \times 2$ such that

$$
\begin{aligned}
g \in N(S) & \Leftrightarrow \quad S g=0 \\
& \Leftrightarrow \quad\left(\mathscr{S} g_{1}, \mathscr{S} g_{2}\right)=0 \\
& \Leftrightarrow \quad \mathscr{S} g_{1}=0, \quad \mathscr{S} g_{2}=0 \\
& \Leftrightarrow \quad g_{1} \in N(\mathscr{S}), \quad g_{2} \in N(\mathscr{S}) \\
& \Leftrightarrow \quad g=\left(\alpha_{1} \nu, \alpha_{2} \nu\right)=\alpha_{1}(\nu, 0)+\alpha_{2}(0, \nu) \\
& \Leftrightarrow \quad g \in \operatorname{span}\{(\nu, 0),(0, \nu)\} .
\end{aligned}
$$

From the basic literature of functional analysis we have very famous result which insures the criteria of denseness of the operators $\mathscr{S}, S, \mathscr{S}^{(\text {adj })}$ and $S^{(\text {adj })}$ in the particular space $\operatorname{span}\{v\}$.

Theorem 4.7. For a bounded linear operator $A: X \rightarrow Y$ between Hilbert spaces $X, Y$ we have

$$
A(X)^{\perp}=N\left(A^{*}\right), \quad N\left(A^{*}\right)^{\perp}=\overline{A(X)} .
$$

Proof. For the first statement, assume that $f \in A(X)^{\perp}$, this means

$$
\begin{aligned}
f \in A(X)^{\perp} & \Leftrightarrow\langle A \phi, f\rangle=0 \text { for all } \phi \in X \\
& \Leftrightarrow\left\langle\phi, A^{*} f\right\rangle=0 \\
& \Leftrightarrow A^{*} f=0 \\
& \Leftrightarrow f \in N\left(A^{*}\right) .
\end{aligned}
$$

For the second part we denote $\mathcal{A}=A(X)$ and obviously we have the closed subspace $\overline{\mathcal{A}} \subset\left(\mathcal{A}^{\perp}\right)^{\perp}$. We take an orthogonal projection $\mathcal{P}$ in $Y$ onto the $\overline{\mathcal{A}}$. Using Theorem 1.25 in [21] we have the orthogonality $\mathcal{P} \phi-\phi \perp\left(\mathcal{A}^{\perp}\right)^{\perp}$ for any arbitrary $\phi \in\left(\mathcal{A}^{\perp}\right)^{\perp}$. But at the same time, because of $\mathcal{A} \subset\left(\mathcal{A}^{\perp}\right)^{\perp}$, we also have $\mathcal{P} \phi-\phi \in \mathcal{A}^{\perp}$. This yield $\mathcal{P} \phi-\phi=0$, i.e., $\mathcal{P} \phi=\phi$ and it follows that $\phi \in \overline{\mathcal{A}}=A(X)$. This gives us the following straightforward result $\overline{\mathcal{A}}=\left(\mathcal{A}^{\perp}\right)^{\perp}$ which together with the first equality gives us the second result. 
Theorem 4.8. Let $\mathscr{S}$ and $\mathscr{S}^{(a d j)}$ be two potential operators defined in equations (4.1.5) and (4.1.11). Then we have the following results.

$$
\overline{R\left(\mathscr{S}^{(a d j)}\right)}=\overline{R\left(\mathscr{S}^{*}\right)}=\operatorname{span}\{\nu\}^{\perp} \quad \text { and } \quad \overline{R(\mathscr{S})}=\operatorname{span}\{\nu\}^{\perp} .
$$

Also the range spaces of operators $S$ and $S^{(a d j)}$ are given by

$$
\begin{aligned}
\overline{R(S)} & =\operatorname{span}\{(\nu, 0),(0, \nu)\}^{\perp}, \\
\overline{R\left(S^{(a d j)}\right)} & =\operatorname{span}\{(\nu, 0),(0, \nu)\}^{\perp} .
\end{aligned}
$$

Proof. From Theorem 4.7 we have the following result

$$
\overline{R\left(\mathscr{S}^{(a d j)}\right)}=N(\mathscr{S})^{\perp} .
$$

Making use of equation (4.1.16) in above equation we proved the first statement of the Lemma, i.e., $\overline{R\left(\mathscr{S}^{(a d j)}\right)}=\operatorname{span}\{v\}^{\perp}$. Now replacing $\mathscr{S}^{(a d j)}$ by $\mathscr{S}$ in equation (4.1.25) we obtain

$$
\overline{R(\mathscr{S})}=N\left(\mathscr{S}^{(a d j)}\right)^{\perp} .
$$

With the help of equation (4.1.18) in above equation we have the second statement of the lemma. Similarly, from equations (4.1.19) and (4.1.20) we can prove the results stated in equations (4.1.23) and (4.1.24).

This is useful result for our analysis, since it shows that for any density $\psi \in$ $\left(L^{2}(\Lambda)\right)^{2 \times 1}$ or $\left(L^{2}(\partial G)\right)^{2 \times 1}$ whose scalar product is zero with the normal vector $\nu$ on the boundary $\Lambda$ or $\partial G$, must belongs to the $\overline{R(\mathscr{S})}$ or $\overline{R\left(\mathscr{S}^{*}\right)}$ respectively. It is discussed before in section 3.1 that $\left(E_{i}, e_{i}\right), i=1,2$, are two linearly independent solution of Oseen equation and $\left(E_{i},-e_{i}\right), i=1,2$ are the two solution of corresponding adjoint Oseen equation. Thus we can see that both solutions are identical for the velocity field however we have difference of minus sign for the pressure term. The continuity equation tells us that

$$
\operatorname{div}_{y} E_{i}(x-y)=0, \quad y \in G \text { and } x \notin \bar{G} \text { for } i=1,2 .
$$

With the help of divergence theorem we have

$$
\left\langle\nu, E_{i}(x-\cdot)\right\rangle_{\left(L^{2}(\partial G)\right)^{2 \times 1}}=0, \quad x \in \mathbb{R}^{2} \backslash \bar{G}, \quad i=1,2 .
$$

Thus, we can find density $g_{i} \in\left(L^{2}(\Lambda)\right)^{2 \times 1}, i=1,2$ such that the approximation

$$
E_{i}(x-y) \approx \int_{\Lambda} E(\xi-y) g_{i}(\xi) d s(\xi), \quad y \in \partial G, \quad i=1,2,
$$

is satisfied in the sense that for a given $x \notin G, \epsilon>0$ there is $g_{i} \in\left(L^{2}(\Lambda)\right)^{2 \times 1}$ such that

$$
\left\|E_{i}(x-\cdot)-\mathscr{S}^{(a d j)} g_{i}\right\|_{\left(L^{2}(\partial G)\right)^{2 \times 1}} \leq \epsilon, \quad i=1,2 .
$$


Lemma 4.9. The tensor $E=\left(E_{1}, E_{2}\right)$ must belongs to $\overline{R(S)}$ and $\overline{R\left(S^{(a d j)}\right)}$.

Proof. The fundamental tensors $E_{i}, i=1,2$, given by the equation (4.1.27), belongs to the spaces $\overline{R(\mathscr{S})}$ and $\overline{R\left(\mathscr{S}^{(a d j)}\right)}$, which means $\left\langle E_{i}, \nu\right\rangle=0, i=1,2$. For any $\beta_{1}, \beta_{2} \in \mathbb{R}$, the tensor $\left(\beta_{1} \nu, \beta_{2} \nu\right)=\beta_{1}(\nu, 0)+\beta_{2}(0, \nu)$ belongs to the null space of $S$ and $S^{(a d j)}$ given in equations (4.1.19), (4.1.20) respectively. Now

$$
\begin{aligned}
\left\langle E,\left(\beta_{1} \nu, \beta_{2} \nu\right)\right\rangle & =\left\langle\left(E_{1}, E_{2}\right),\left(\beta_{1} \nu, \beta_{2} \nu\right)\right\rangle \\
& =\beta_{1}\left\langle E_{1}, \nu\right\rangle+\beta_{2}\left\langle E_{2}, \nu\right\rangle \\
& =0
\end{aligned}
$$

With the help of Theorem 4.8 and above result (4.1.29) we can conclude that the tensor $E$ belong to $\overline{R(S)}$ and $\overline{R\left(S^{(a d j)}\right)}$.

With all this theory we are now in position to approximate the point source $E(x-\cdot)$ on the boundary of the approximation domain $G$ with a particular integral on $\Lambda$.

Theorem 4.10. Let $(E, e)$ be the fundamental solution of the Oseen equation. Then we can approximate

$$
E(x-y) \approx \int_{\Lambda} E(\xi-y) g(\xi) d s(\xi), \quad y \in \partial G
$$

in the sense that for given $x \notin G, \epsilon>0$ there is $g \in\left(L^{2}(\Lambda)\right)^{2 \times 2}$ such that

$$
\left\|E(x-\cdot)-S^{(a d j)} g\right\|_{\left(L^{2}(\partial G)\right)^{2 \times 2}} \leq \epsilon .
$$

Proof. With the help of equation (4.1.27) and Lemma 4.9 we have the statement of the theorem.

Remark: As a remark we would like to mention that due to the wellposedness of the adjoint Oseen equation in the interior domain (see the remark after Corollary 3.20) and the estimates (3.2.66), we have the following approximation

$$
(\nabla \Phi(x-y))^{T} \approx \int_{\Lambda}(\nabla \Phi(\xi-y))^{T} g(\xi) d s(\xi) \quad y \in G
$$

in the sense of

$$
\left\|P^{(a d j)} g-(\nabla \Phi(x-\cdot))^{T}\right\|_{\left(L^{2}(\partial G)\right)^{2 \times 2}} \leq \epsilon .
$$




\subsection{Uniqueness of Inverse problem}

As it is discussed before that our inverse problem is to reconstruct the flow field and to determine the unknown boundary from the knowledge of $u$ on $\Lambda$. For this we consider the weak solution of the Oseen equation

$$
\mu \Delta u-\partial_{1} u-\operatorname{grad} p=0, \quad \operatorname{div} u=0
$$

in the exterior of the unknown obstacle by taking the following homogeneous boundary condition

$$
u=0 \quad \text { on } \quad \partial D
$$

and the condition at infinity is

$$
\lim _{x \rightarrow \infty} u=u_{\infty}=(1,0) .
$$

Before establishing the point source method we are interested in the uniqueness result of the above inverse problem. Kress and Meyer [23] developed these uniqueness results in 2000 , we adopt these results in our analysis. For convenience of the reader we present these results here, explicitly.

Lemma 4.11. Let $\Omega$ be a bounded domain in $\mathbb{R}^{2}$. Assume that $u=0$ on $\partial \Omega$ with $u \in W^{1}(\Omega) \cap C^{1}(\Omega) \cap C(\bar{\Omega})$. Then there exists a sequence $u_{n} \in C_{0}^{1}(\Omega)$ such that

$$
\left\|u_{n}-u\right\|_{W^{1}(\Omega)} \rightarrow 0, \quad n \rightarrow \infty,
$$

or in other words $u \in W_{0}^{1}(\Omega)$

Proof. See Lemma 3.1 in [23]

Theorem 4.12. Let us consider $D_{1}$ and $D_{2}$ are two domains contained in the interior of a measurement boundary $\Lambda$. Assume that we have two weak solutions of the boundary value problem (4.2.1)-(4.2.3) for $D_{1}$ and $D_{2}$ respectively. If these solutions have the same value at $\Lambda$ then $D_{1}=D_{2}$.

Proof. Following Kress and Meyer [23], we assume to the contrary that $D_{1} \neq D_{2}$ and the solutions $u_{1}=u_{2}$ to the corresponding boundary value problem coincides on $\Lambda$. From this by the uniqueness of the solution of Dirichlet problem, applied to the exterior of $\Lambda$, we have $u_{1}=u_{2}$ in the exterior of $\Lambda$. As our solutions are represented in terms of single layer potential so it can easily deduced that $u_{1}$ and $u_{2}$ are analytic in the exterior of the respective domains i.e., $\mathbb{R}^{2} \backslash \bar{D}_{1}$ and $\mathbb{R}^{2} \backslash \bar{D}_{2}$. By the analyticity and the uniqueness result we can say that $u_{1}=u_{2}$ in the whole region except $D_{1} \cup D_{2}$. Let us cal this unbounded region by $G$.

Now define another region $G^{*}:=\left(\mathbb{R}^{2} \backslash G\right) \backslash \bar{D}_{2}$ and assume that it is non-empty. Now the solution $u:=u_{2}$ is defined in the region $G^{*}$ as $u_{2}$ is the solution of equation 
(4.2.1)-(4.2.3) for the exterior of $D_{2}$. Also $u=0$ on $\partial G^{*}$ in the classical sense because $u=0$ on $\partial D_{2}$ which is apart of $\partial G^{*}$.

By the measure theory, the intersection of measurable sets is measurable. This implies $G^{*}$ is a measurable set. According to equation (3.1.9) we have, $u_{2} \in$ $W_{\text {loc }}^{1}\left(\mathbb{R}^{2} \backslash D_{2}, \mathbb{R}^{2}\right)$ and $p \in L_{\text {loc }}^{2}\left(\mathbb{R}^{2} \backslash D_{2}, \mathbb{R}\right)$ this implies that $u \in W^{1}\left(G^{*}, \mathbb{R}^{2}\right)$ and $p \in L^{2}\left(G^{*}, \mathbb{R}^{2}\right)$ and hence finally we can conclude that $\operatorname{grad} u \in W^{1}\left(G^{*}, \mathbb{R}^{2}\right)$. This means that, from Lemma 4.2 , there exist a sequence $u_{n} \in C_{0}^{1}\left(G^{*}, \mathbb{R}^{2}\right)$ such that $\left\|u_{n}-u\right\| \rightarrow 0, n \rightarrow \infty$.

Now applying the divergence theorem on the Oseen equation for $u$, we obtain

$$
\int_{G^{*}}\left\{\mu \nabla u \cdot \nabla u_{n}+\partial_{1} u \cdot u_{n}-p \operatorname{div} u_{n}\right\} d x=0 .
$$

The last terms vanishes when $n \rightarrow \infty$ since div $u=0$. The second term vanishes because

$$
\int_{G^{*}} \partial_{1} u \cdot u_{n}=-\int_{G^{*}} u \cdot \partial_{1} u_{n}
$$

So after passing the limit $n \rightarrow \infty$ in equation (4.2.4) we are left with

$$
\int_{G^{*}}|\nabla u|^{2} d x=0 .
$$

This implies that $u$ must be a constant and from the boundary condition (4.2.2) we have $u=u_{2}=0$ in $D^{*}$. By analyticity it follows that $u_{2}=0$ in $\mathbb{R}^{2} \backslash D_{2}$ which is a contradiction to the condition (4.2.3), since $u_{\infty} \neq 0$ and with this our proof is complete.

\subsection{The Point Source Method for Oseen Flow}

In this section we develop the point source method for the reconstruction of flow field and to locate and reconstruct the unknown obstacle $D$. Later on we investigate the convergence of the method. In particular we carry out the explicit construction of a kernel or function $g_{x} \in\left(L^{2}(\Lambda)\right)^{2 \times 2}$ for $x \notin G \backslash D$, such that the velocity field $u(x)$ in $x$ is approximated by the back projection formula

$$
u_{r e c}(x) \approx \int_{\Lambda} g_{x}^{T}(\xi)\left(u_{\text {meas }}(\xi)-u_{\infty}\right) d s(\xi)+u_{\infty}, \quad x \in \mathbb{R}^{2} \backslash D,
$$

where $g_{x}(\xi)=\left(g_{x, 1}(\xi), g_{x, 2}(\xi)\right)$ is a tensor with unknown densities

$$
g_{x, 1}(\xi)=\left(\begin{array}{c}
g_{x, 1,1}(\xi) \\
g_{x, 1,2}(\xi)
\end{array}\right), \quad g_{x, 2}(\xi)=\left(\begin{array}{c}
g_{x, 2,1}(\xi) \\
g_{x, 2,2}(\xi)
\end{array}\right) .
$$

Since in the inverse problem we have to construct the size and the location of the obstacle $D$ as well as the flow field. In the point source method we replace our 
unknown domain $D$ by a known domain $G$. We call this domain as an approximation domain. Our whole analysis depends on the choice of approximation domain. There are several methods in literature (see $[6,10,19,20]$ ) for example the needle approach, domain sampling or newly develop LASSO scheme. We will discuss in detail about the choice of approximation domain in numerical part of this monograph.

From Theorem 4.10 we know that the fundamental solution $E(x-\cdot), x \notin \bar{G}$ can be approximated by the single layer potential operator corresponding to each element $x$ in the grid such that

$$
S^{(a d j)} g_{x} \approx E(x-\cdot) \quad \text { on } \quad \partial G .
$$

Since the above equation is ill-posed in nature so we used Tikhonov regularization (see details in section 2.4) to replace the above approximation with the following regularized equation,

$$
\left(\alpha I+S S^{(a d j)}\right) g_{x}=S E(x-\cdot),
$$

to get the approximate solution $g_{x}$ which satisfies

$$
\left\|S^{(a d j)} g_{x}-E(x-\cdot)\right\|_{L^{2}(\partial G)} \leq \epsilon .
$$

Here the important question is how to choose the regularization parameter $\alpha$. For this we assume that $g_{x}$ is the unique solution of the equation (4.3.4) and we define a function $\mathcal{F}(\alpha)$ such that

$$
\mathcal{F}(\alpha):=\left\|S^{(a d j)} g_{x}-E(x-\cdot)\right\|-\epsilon^{2}
$$

for any $0<\epsilon<\|E(x-\cdot)\|$. It can be shown by standard arguments that for $\alpha \rightarrow 0$ in equation (4.3.4) we have $\left\|S^{(a d j)} g_{x}-E(x-\cdot)\right\| \rightarrow 0$, and for $\alpha \rightarrow \infty$ we have $S^{(a d j)} g_{x} \rightarrow 0$. Thus equation (4.3.6) reduces to

$$
\mathcal{F}(\alpha)=\left\{\begin{array}{lll}
\|E(x-\cdot)\|-\epsilon^{2}>0 & \text { for } & \alpha \rightarrow \infty \\
-\epsilon^{2}<0 & \text { for } & \alpha \rightarrow 0
\end{array} .\right.
$$

Thus $\mathcal{F}(\alpha)$ has zeros $\alpha=\alpha(\epsilon)$ or in other words

$$
\mathcal{F}(\alpha)=\left\|S^{(a d j)} g_{x}-E(x-\cdot)\right\|-\epsilon^{2}=0
$$

Substituting the value of $g$ from equation (4.3.4) in the above one, we have the value of $\alpha$ from the following equation

$$
\left\|S^{(a d j)}\left(\alpha I+S S^{(a d j)}\right)^{-1} S E(x-\cdot)-E(x-\cdot)\right\|-\epsilon=0 .
$$

However in this monograph, instead of using this procedure we choose $\alpha$ by trial and error. It is observed that we have a good approximation from $\alpha=10^{6}$ to $\alpha=10^{9}$. 
To construct the back projection formula for the reconstruction of the velocity field and the shape of unknown obstacle for the Oseen equation we use the following potential representation

$$
u(x)=(\mathscr{S} \varphi)(x)+\gamma \nabla \Phi(x)+u_{\infty},
$$

where $\gamma=\langle\varphi, \nu\rangle$. Here we use the single layer potential operator,

$$
(\mathscr{S} \varphi)(x)=\int_{\partial D} E(x-y) \varphi(y) d s(y), \quad y \in \partial D,
$$

for $D \subset G$. We choose this special type of potential representation since it solves the Oseen equation (compare Theorem 3.9). Now from Theorem 4.10, the fundamental solution $E(x-\cdot)$ can be approximated by the single layer potential at the source point $x$ under the norms described in equation (4.1.31). Taking the transpose of the equation (4.1.30) we have

$$
E^{T}(x-y) \approx\left(\int_{\Lambda} E(\xi-y) g_{x}(\xi) d s(\xi)\right)^{T} \quad y \in \partial D .
$$

Due to the symmetry of fundamental solution $E(x-\cdot)$ we are left with

$$
E(x-y) \approx \int_{\Lambda} g_{x}^{T}(\xi) E(\xi-y) d s(\xi), \quad y \in \partial D
$$

Substituting the value of equation (4.3.12) into equation (4.3.10), we obtain

$$
(\mathscr{S} \varphi)(x) \approx \int_{\partial D}\left(\int_{\Lambda} g_{x}^{T}(\xi) E(y-\xi) d s(\xi)\right) \varphi(y) d s(y)
$$

The inequality (4.3.11) is understood in the sense that given $x \in G, \epsilon>0$ there is $g_{x} \in\left(L^{2}(\Lambda)\right)^{2 \times 2}$ such that

$$
\left\|(\mathscr{S} \varphi)(x)-\int_{\partial D}\left(\int_{\Lambda} g_{x}^{T}(\xi) E(y-\xi) d s(\xi)\right) \varphi(y) d s(y)\right\|_{L^{2}(\partial G)} \leq \epsilon .
$$

Interchanging the integrals in equation (4.3.13) we derive

$$
\begin{aligned}
& (\mathscr{S} \varphi)(x) \approx \int_{\Lambda} g_{x}^{T}(\xi) \underbrace{\int_{\partial D} E(\xi-y) \varphi(y) d s(y)}_{(\mathscr{S} \varphi)(\xi)} d s(\xi) . \\
& (\mathscr{S} \varphi)(x) \approx \int_{\Lambda} g_{x}^{T}(\xi)(\mathscr{S} \varphi)(\xi) d s(\xi),
\end{aligned}
$$

in the sense of the following norm

$$
\left\|(\mathscr{S} \varphi)(x)-\int_{\Lambda} g_{x}^{T}(\xi)(\mathscr{S} \varphi)(\xi) d s(\xi)\right\|_{L^{2}(\partial G)} .
$$


On the boundary $\Lambda$ we have the measured velocity field $u_{\text {meas }}$, we employ equation (4.3.9) to transform (4.3.15) into

$$
\begin{aligned}
(\mathscr{S} \varphi)(x) \approx & \int_{\Lambda} g_{x}^{T}(\xi)\left[u_{\text {meas }}(\xi)-\gamma \nabla \Phi(\xi)-u_{\infty}\right] d s(\xi) \\
= & \int_{\Lambda} g_{x}^{T}(\xi)\left(u_{\text {meas }}-u_{\infty}\right)(\xi) d s(\xi) \\
& -\gamma \int_{\Lambda} g_{x}^{T}(\xi) \nabla \Phi(\xi) d s(\xi) .
\end{aligned}
$$

Substituting the value of equation (4.3.17) into equation (4.3.9)

$$
\begin{aligned}
u_{r e c}(x) \approx & \int_{\Lambda} g_{x}^{T}(\xi)\left(u_{\text {meas }}-u_{\infty}\right)(\xi) d s(\xi) \\
& -\gamma\left(\int_{\Lambda} g_{x}^{T}(\xi) \nabla \Phi(\xi) d s(\xi)-\nabla \Phi(x)\right)+u_{\infty}
\end{aligned}
$$

Taking transpose of equation (4.1.32), we obtain

$$
\nabla \Phi(x) \approx \int_{\Lambda} g(\xi)^{T} \nabla \Phi(\xi) d s(\xi)
$$

in the sense of equation (4.1.33). Finally we have the reconstruction formula

$$
u_{r e c}(x) \approx \int_{\Lambda} g_{x}^{T}(\xi)\left(u_{\text {meas }}(\xi)-u_{\infty}\right) d s(\xi)+u_{\infty}
$$

in the sense that given $\epsilon>0$ there is $g_{x}^{T} \in\left(L^{2}(\Lambda)\right)^{2}$ such that

$$
\left\|u_{\text {rec }}-\int_{\Lambda} g_{x}^{T}(\xi)\left(u_{\text {meas }}(\xi)-u_{\infty}\right) d s(\xi)+u_{\infty}\right\|_{L^{2}(\partial G)} \leq \epsilon .
$$

We summarize the results in the following theorem.

Theorem 4.13. For the test domain $G$ with $D \subset G$ and $x \in \mathbb{R}^{2} \backslash \bar{G}$, the reconstructed velocity field defined by

$$
u_{r e c}(x):=\int_{\Lambda} g_{x}^{T}(\xi)\left(u_{m e a s}(\xi)-u_{\infty}\right) d s(\xi)+u_{\infty}
$$

with a density $g$ satisfying

$$
\left\|S^{*} g_{x}-E(x-\cdot)\right\|_{L^{2}(\partial G)} \leq \epsilon
$$

approximates the true velocity field uniformly on $\mathbb{R}^{2} \backslash \bar{G}$ such that

$$
\lim _{\epsilon \rightarrow 0} \max _{x \in \mathbb{R}^{2} \backslash \bar{G}}\left|u(x)-u_{\text {rec }}(x)\right|=0 .
$$




\section{Chapter 5}

\section{Tests for Analytical Continuation}

The goal of this chapter is to analyze methods which test for analytic extensions in fluid dynamics. We will discuss and analyze three different schemes. First, we describe and analyze the no-response test (Luke and Potthast 2003) [27] and the range test (Kusiak, Potthast and Sylvester 2003), [41] which have been suggested for acoustic and electromagnetic problems. We will prove the convergence of the methods when applied to the Oseen equation. In particular, we will provide a new approach to show convergence of the no-response test.

Then, we describe an alternative way to determine analytic extensibility via a convergence test. We describe such a test based on the point source method (Potthast 1996). Finally, we provide a convergence proof of the convergence test.

In section 4.1 of Chapter 4 , we discuss in detail about the single layer potential operators, $\mathscr{S}$ and $\mathscr{S}^{(a d j)}$, of the Oseen equation (3.1.1) and the adjoint Oseen equation (3.1.10). We also analyze the basic properties of these potential operators. Here we recall the potential operators for the convenience of the reader. The single-layer potential operator for the Oseen equation (3.1.1) over a $C^{1}$-surface $\partial G$ is given by

$$
\left(\mathscr{S}_{\partial G} \varphi\right)(x)=\int_{\partial G} E(x-y) \varphi(y) d s(y), \quad x \in \Lambda .
$$

And the single-layer potential operator for the adjoint Oseen equation (3.1.10) is

$$
\left(\mathscr{S}_{\Lambda}^{(a d j)} \psi\right)(y)=\int_{\Lambda} E(x-y) \psi(x) d s(x), \quad x \in \partial G .
$$

Furthermore it is observed that for the appropriate choice of domains $G$ and $\Omega$ the operator $\mathscr{S}^{(\text {adj })}$ behaves like the adjoint operator of $\mathscr{S}$. As a remark we would like to mention the range spaces of the operators $\mathscr{S}$ and $\mathscr{S}^{(\text {adj })}$

$$
\overline{R(\mathscr{S})}=\operatorname{span}\{\nu\}^{\perp}, \quad \overline{R\left(\mathscr{S}^{(a d j)}\right)}=\overline{R\left(\mathscr{S}^{*}\right)}=\operatorname{span}\{\nu\}^{\perp}
$$


here $\nu$ is a unit outward normal vector.

\section{$5.1 \quad$ Range Test}

In the theory of Inverse problems, the study of the reconstruction of the shape of unknown obstacles is of great mark. In last two decades there are several methods developed for the reconstruction of the unknown obstacle. In 2003 Potthast, Sylvester and Kusiok [41] introduced range test to locate and reconstruct the unknown object in scattering problems. A more comprehensive study about the range test on inverse scattering theory is done by Schulz in his $\mathrm{PhD}$ thesis [42].

Here, we present new aspects of the range test in inverse fluid flow problems. Consider a test domain $G$ and the single layer potential operator $\mathscr{S}$ defined in equation (5.0.1). We have the measured velocity field denoted by $u_{\text {meas }}-u_{\infty}$ on $\Lambda$. The basic idea of the range test is to check the analytical extensibility of a potential operator $\mathscr{S}_{\partial G}$ into $\mathbb{R}^{2} \backslash G$ by investigating the solvability of an ill-posed equation of the type

$$
\mathscr{S}_{\partial G} \varphi=u_{\text {meas }}-u_{\infty}
$$

where $u_{\text {meas }} \in L^{2}(\Lambda)$. If the equation (5.1.1) is solvable with $\varphi \in L^{2}(\partial G)$, then we have the analytically extended field into the region $\mathbb{R}^{2} \backslash G$ with boundary values in $W^{1}(\partial G)$. We collect all these extensions in a set which is obtained by the analytical extension of the measured velocity field $u_{\text {meas }}$. The complement of this set is a subset of the unknown obstacle $D$.

The methodology to check the solvability for an ill-posed equation for the scattering theory is given in Theorem 3.5 of [41]. However for special setting for the potential operators of the Oseen equation, since they are not injective in general, we present the following theorem to test the solvability of an ill-posed equation (5.1.11).

Theorem 5.1. Assume that $G$ be a bounded domain. We consider the single layer potential operator defined in equation (5.0.1) from the Hilbert space $X:=$ $\operatorname{span}\{\nu\}^{\perp} \subset L^{2}(\partial G)$ to $Y:=\operatorname{span}\{\nu\}^{\perp} \subset L^{2}(\Lambda)$. Then for the Tikhonov solution

$$
\varphi_{\alpha}:=\left(\alpha I+\mathscr{S}^{*} \mathscr{S}\right)^{-1} \mathscr{S}^{*} f
$$

with regularization parameter of the equation

$$
\mathscr{S} \varphi=f
$$

such that $\langle f, \nu\rangle=0$ and $f \in W^{1}(\Omega)$, we obtain the behavior

$$
\lim _{\alpha \rightarrow 0}\left\|\varphi_{\alpha}\right\|= \begin{cases}\infty, & \text { if } f \notin \mathscr{S}(X) \\ \left\|\varphi^{*}\right\|<\infty, & \text { if } \mathscr{S} \varphi^{*}=f\end{cases}
$$


Proof. First, assume that $f \notin \mathscr{S}(X)$. Furthermore we assume that there is a constant $C$ such that the density $\varphi_{\alpha}$ is bounded for sufficiently small $\alpha>0$. Then there is a sequence $\varphi_{k} \rightarrow 0$ for $k \rightarrow \infty$ such that the weak convergence $\varphi_{\alpha_{k}} \rightarrow \tilde{\varphi}, k \rightarrow \infty$ holds. Thus the linear single layer potential operator maps the weakly convergence sequence into a strongly convergence sequence, i.e., we obtain

$$
\mathscr{S} \varphi_{\alpha_{k}} \rightarrow \mathscr{S} \tilde{\varphi}=: \tilde{f}
$$

with some $\tilde{f} \in \mathscr{S}(X) \subset Y$ with $\langle\tilde{f}, \nu\rangle=0$. The regularized version of equation (5.1.3) for the densities $\varphi_{\alpha_{k}}$ is

$$
\left(\alpha_{k} I+\mathscr{S}^{*} \mathscr{S}\right) \varphi_{\alpha_{k}}=\mathscr{S}^{*} f
$$

Now applying the limit $k \rightarrow \infty$ on equation (5.1.6), we obtain,

$$
\mathscr{S}^{*} \mathscr{S} \tilde{\varphi}=\mathscr{S}^{*} f .
$$

From (5.1.6) we have $\mathscr{S}^{*} \tilde{f}=\mathscr{S}^{*} f$, and both $f$ and $\tilde{f}$ are in $\operatorname{span}\{\nu\}^{\perp}$. However, the operator $\mathscr{S}^{*}$ restricted to $\operatorname{span}\{\nu\}^{\perp}$ is injective, which yields $\tilde{f}=f$. This observation leads us to the contradiction that $f \in \mathscr{S}(A)$ since $\tilde{f} \in \mathscr{S}(X)$. This proves the first line of equation (5.1.4).

To prove the second line of equation (5.1.4), assume that $f \in \mathscr{S}(X)$, then there must be some density $\varphi^{*} \in X$ such that

$$
\mathscr{S} \varphi^{*}=f .
$$

Now applying the limit $\alpha \rightarrow 0$ on both side of equation (5.1.2) and using equation (5.1.8), we obtain

$$
\lim _{\alpha \rightarrow 0} \varphi_{\alpha}=\lim _{\alpha \rightarrow 0}\left(\alpha I+\mathscr{S}^{*} \mathscr{S}\right)^{-1} \mathscr{S}^{*} \mathscr{S} \varphi^{*}
$$

Since $f \in \mathscr{S}(X)=\operatorname{span}\{\nu\}^{\perp}$ so we can say that the operator $\mathscr{S}$ is injective in a proper subspace of $X$, i.e., $\operatorname{span}\{\nu\}^{\perp}$, and therefore we can apply Theorem 2.26 on equation (5.1.9) such that

$$
\lim _{\alpha \rightarrow 0} \varphi_{\alpha}=\lim _{\alpha \rightarrow 0} R_{\alpha} \mathscr{S} \varphi^{*}
$$

With the help of Definition 2.19, we have

$$
\lim _{\alpha \rightarrow 0} \varphi_{\alpha}=\varphi^{*} .
$$

This proves the second statement of the equation (5.1.4).

In above theorem, equation (5.1.4) suggests that if the equation (5.1.1) is solvable then the norm $\left\|\varphi_{\alpha}\right\|$ is finite with $\alpha \rightarrow 0$, while on the other hand we say that the 
equation is unsolvable if $\left\|\varphi_{\alpha}\right\| \rightarrow \infty$. We can use this behavior of norm density for analyzing the extensibility of the velocity field from $\Lambda$ to $\Omega \backslash G$. First we calculate the norm $\left\|\varphi_{\alpha}\right\|$ for solutions with a test domain $G$. If $\left\|\varphi_{\alpha}\right\|$ is less than or equal to a suitable cut off parameter $\kappa$ for a small regularization parameter $\alpha$ then we say that the equation (5.1.4) is solvable and hence the velocity field is analytical extensible in $\Omega \backslash G$.

Before presenting the key result for the range test we need to check the solvability of the equation (5.1.1) in $L^{2}(\partial G)$. We would like to mention that the operator $\mathscr{S}$ maps from $L^{2}(\partial G)$ to $L^{2}(\Lambda)$. We are interested in the case when the values at the boundary $\Lambda$ can be extended to the $\partial G$.

Lemma 5.2. Consider the boundary values in $W^{1}(\partial G)$ and perpendicular to the $\operatorname{span}\{\nu\}$ then the equation

$$
\mathscr{S} \varphi=u_{\text {meas }}-u_{\infty}
$$

has a solution in $L^{2}(\partial G)$.

Proof. We know that the operator $\mathscr{S}$ maps from $W^{-1 / 2}(\partial G)$ to $W^{1 / 2}(\partial G)$ and we know that the space $W^{1}(\partial G)$ is a subset of $W^{1 / 2}(\partial G)$. Then with the help of Corollary 3.21 we have the solution of the equation (5.1.12) in $W^{-1 / 2}(\partial G)$.

Now we decompose the operator $\mathscr{S}$ such that

$$
\mathscr{S}=\mathscr{S}_{0}+\left(\mathscr{S}-\mathscr{S}_{0}\right)
$$

where $\mathscr{S}_{0}$ is the principle part of the parametrized version of operator $\mathscr{S}$ on the interval $[0,2 \pi]$ and it is defined in equation (3.2.29). Thus equation (5.1.12) can be written as

$$
\mathscr{S}_{0} \varphi+\left(\mathscr{S}-\mathscr{S}_{0}\right) \varphi=u_{\text {meas }}-u_{\infty}
$$

or

$$
\varphi=\mathscr{S}_{0}^{-1}\left(u_{\text {meas }}-u_{\infty}\right)-\mathscr{S}_{0}^{-1}\left(\mathscr{S}-\mathscr{S}_{0}\right) \varphi
$$

The second term of the right hand side of equation (5.1.13) is in $W^{1}(\partial G)$, because $\mathscr{S}-\mathscr{S}_{0}$ maps $W^{r}[0,2 \pi]$ to $W^{r+2}[0,2 \pi], r \in \mathbb{R}$ (compare the proof of Lemma 3.8$)$, and the first term is in $L^{2}(\partial G)$ because of the mapping properties of operator $\mathscr{S}_{0}$ given in equation (3.2.30). Thus we have the solvability of equation (5.1.12) with $\varphi \in L^{2}(\partial G)$.

Now we are ready to present the essential result for the range test.

Theorem 5.3. The equation (5.1.1) is solvable for some domain $G \subset \Omega$ if and only if $u_{\text {meas }} \in L^{2}(\Lambda)$ can be analytically extended into $\Omega \backslash G$ with boundary values in $W^{1}(\partial G)$. 


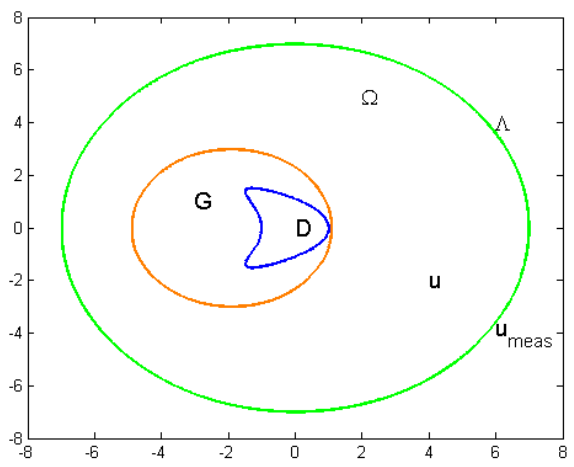

Figure 5.1: This figure exhibits the setting of our approximation domain $D \subset G$ inside $\Omega$. We have measured values of velocity field at $\Lambda$, boundary curve of $\Omega$, and in both the range test and the no-response test our primary interest is to observe that this measured velocity field is analytically extensible in $\Omega \backslash G$.

Proof. Assume first that $u$ can be analytically extended into the exterior of the domain $G$ and the neighborhood of $\partial G$, i.e., $\Omega \backslash G$. We can solve the equation (5.1.1) on the boundary of domain $G$ which has a solution since $\mathscr{S}$ maps $L^{2}(\partial G)$ to $W^{1}(\partial G)$, (compare Lemma 5.2) and by the solution of exterior Dirichlet problem for Oseen flow for the domain $G$ it coincides on $\mathbb{R}^{2} \backslash G$.

Conversely, we assume that the equation (5.1.1) has a solution in $L^{2}(\partial G)$, then clearly $\mathscr{S} \varphi$ defines the desired analytical extension with the boundary values in $W^{1}(\partial G)$. This completes the proof.

In his thesis [42], Schulz observed in scattering theory that it is almost impossible to reconstruct the full shape of the obstacle with the help of one incident wave coming from one side. However he suggests that we obtain good reconstruction of the unknown obstacles with the help of a multiwave range test. Since the direction of flow field is fixed in this monograph i.e., from left to right. Thus in the view of Schulz analysis and due to the wake region behind the obstacle we only obtain a subset of the unknown obstacle. This is a major drawback of the range test, however to some extent it gives us the location of the object.

\subsection{No Response Test}

In this section we study the no-response test, which is proposed by the Luke and Potthast in 2003 [27], and it belongs to the class of probe and sampling techniques for the inverse problems. Initially, this methodology was introduced in the area of inverse scattering to reconstruct the shape of unknown scatter in a test domain. The basic idea of the method is to examine that the unknown scatterer lies inside 
some test domains or not. We examine this by constructing the set of incident fields that are small on test domains and large outside. With the help of far field data we can calculate the far field pattern (responses) corresponding to these incident fields. If these responses are small then the unknown scatterer lies inside the union of all test domains. Also with the help of these responses we also observe the analytical extension of the field. Since small responses indicate the location of the unknown scatterer therefore we call this method no response test. Later on in his Phd thesis Kühn [24] implements the no response test on magnetic tomography, where domains of distinct conductivity within some conductor are reconstructed with the help of boundary measurements of the magnetic and electric fields. In 2007 Potthast [39] presents a convergence proof for the no response test. In the same article [39], Potthast suggests a multiwave version of the no response test and proves its convergence.

With this brief review, we observed that the no response test is basically a very significant method in inverse scattering and inverse electromagnetic theory to reconstruct the unknown obstacles and to observe the analytical continuation of the field. Here in this monograph we analyze the applicability of the no response test in the theory of inverse fluid flow problem.

For the no response test we assume that a measurement $u_{\text {meas }} \in\left(L^{2}(\Lambda)\right)^{2 \times 1}$ of a flow field $u$ is given on the measurement surface $\Lambda$ as described in section 5.1. Consider a test domain $G \subset \Omega$. The goal of the test is to evaluate whether the flow $u$ can be analytically extended into $\Omega \backslash G$.

The no response test investigates integrals of the type

$$
\mathfrak{r}(g):=\left\|\int_{\Lambda} g^{T}(y)\left(u_{\text {meas }}(y)-u_{\infty}\right) d s(y)\right\|, g \in\left(L^{2}(\Lambda)\right)^{2 \times 2},
$$

under the condition that

$$
\left\|\int_{\Lambda} E(y-\cdot) g(y) d s(y)\right\|_{\left(L^{2}(\partial G)\right)^{2 \times 2}} \leq 1 .
$$

We call $\mathfrak{r}(g)$ the response of the system for an excitation

$$
v(x)=\int_{\Lambda} E(y-x) g(y) d s(y), \quad x \in \Omega .
$$

We define

$$
\mathfrak{T}(G):=\sup \left\{\mathfrak{r}(g): \quad g \in\left(L^{2}(\Lambda)\right)^{2}, g \text { satisfies (5.2.2) }\right\}
$$

and call it the maximal response for $G$ of the system. It describes the maximal response for excitations bounded on $G$ by 1 .

The expression (5.2.3) can be written in the potential form as

$$
\left(\mathscr{S}^{*} g\right)(x)=v(x), \quad x \in \Omega \text { and } g \in\left(L^{2}(\Lambda)\right)^{2 \times 2} .
$$


This equation (5.2.5) together with equation (5.1.1) are ill-posed in nature and to solve these equations we need to introduce some regularization technique. From Theorem 4.8 we know that the potential operators $\mathscr{S}$ and $\mathscr{S}^{*}$ have a dense range in the $\operatorname{span}\{\nu\}^{\perp}$. This means that if we have $\left(u-u_{\infty}\right) \in \operatorname{span}\{\nu\}^{\perp}$ and $v \in \operatorname{span}\{\nu\}^{\perp}$ then our single layer potential operators $\mathscr{S}$ and $\mathscr{S}^{*}$ are injective in their respective spaces.

Theorem 5.4. Consider the following single layer potential operators,

$$
\begin{aligned}
\mathscr{S} & : \operatorname{span}\{\nu\}^{\perp} \subset L^{2}(\partial G) \rightarrow L^{2}(\Lambda), \\
\mathscr{S}^{*} & : \operatorname{span}\{\nu\}^{\perp} \subset L^{2}(\Lambda) \rightarrow L^{2}(\partial G),
\end{aligned}
$$

which are injective and adjoint to each other. Furthermore, assume that $\varphi \in$ $\operatorname{span}\{\nu\}^{\perp} \subset L^{2}(\partial G)$ and $\psi \in \operatorname{span}\{\nu\}^{\perp} \subset L^{2}(\Lambda)$ and with the help of Tikhonov regularization scheme, we have

$$
\begin{aligned}
& \mathfrak{R}_{\alpha}:=\left(\alpha I+\mathscr{S}^{*} \mathscr{S}\right)^{-1} \mathscr{S}^{*}, \\
& \mathfrak{R}_{\alpha}^{*}:=\left(\alpha I+\mathscr{S} \mathscr{S}^{*}\right)^{-1} \mathscr{S},
\end{aligned}
$$

then the following convergence properties,

$$
\begin{aligned}
\mathfrak{R}_{\alpha} \mathscr{S} \varphi & \rightarrow \varphi \text { for } \alpha \rightarrow 0, \\
\mathfrak{R}_{\alpha}^{*} \mathscr{S}^{*} \psi & \rightarrow \psi \text { for } \alpha \rightarrow 0 .
\end{aligned}
$$

holds.

Lemma 5.5. Let $\mathscr{A}$ and $\mathscr{A}^{*}$ are two adjoint compact linear operators and $\alpha>0$ be a regularization parameter. Then we have

$$
\mathscr{A}\left(\alpha I+\mathscr{A}^{*} \mathscr{A}\right)^{-1}=\left(\alpha I+\mathscr{A} \mathscr{A}^{*}\right)^{-1} \mathscr{A} .
$$

Proof. If we multiply the last equation (5.2.10) from the left with $\left(\alpha I+\mathscr{A}^{*} \mathscr{A}^{*}\right)$ and from the right with $\left(\alpha I+\mathscr{A}^{*} \mathscr{A}\right)$ then the proof is straightforward.

Remark: As a remark we would like to mention that with the following small calculation, $\mathfrak{R}_{\alpha}$ and $\mathfrak{R}_{\alpha}^{*}$ are adjoint to each others, i.e.,

$$
\begin{aligned}
\left\langle\mathfrak{R}_{\alpha} \varphi, \psi\right\rangle & =\left\langle\left(\alpha I+\mathscr{S}^{*} \mathscr{S}\right)^{-1} \mathscr{S}^{*} \varphi, \psi\right\rangle \\
& =\left\langle\mathscr{S}^{*} \varphi,\left(\left(\alpha I+\mathscr{S}^{*} \mathscr{S}\right)^{-1}\right)^{*} \psi\right\rangle \\
& =\left\langle\mathscr{S}^{*} \varphi,\left(\alpha I+\mathscr{S}^{*} \mathscr{S}\right)^{-1} \psi\right\rangle \\
& =\left\langle\varphi, \mathscr{S}\left(\alpha I+\mathscr{S}^{*} \mathscr{S}\right)^{-1} \psi\right\rangle \\
& =\left\langle\varphi,\left(\alpha I+\mathscr{S} \mathscr{S}^{*}\right)^{-1} \mathscr{S} \psi\right\rangle \\
& =\left\langle\varphi, \mathfrak{R}_{\alpha}^{*} \psi\right\rangle .
\end{aligned}
$$

The second last line of equation (5.2.11) is justified because of Lemma 5.5. 
Definition 5.6. We define a range test functional $\mathfrak{T}_{\alpha, r t}$ such that

$$
\mathfrak{T}_{\alpha, r t}:=\left\|\varphi_{\alpha}\right\|,
$$

where $\varphi_{\alpha}=\mathfrak{R}_{\alpha}\left(u_{\text {meas }}-u_{\infty}\right)$. Furthermore we define

$$
\mathfrak{T}_{r t}:=\lim _{\alpha \rightarrow 0} \mathfrak{T}_{\alpha, r t}
$$

Remark: We remark that for Hilbert spaces the following expression

$$
\left\|\varphi_{\alpha}\right\|=\sup _{\|v\| \leq 1}\left\langle v, \varphi_{\alpha}\right\rangle
$$

is valid in general.

We can also write the no response test functional, $\mathfrak{T}_{\alpha, n r t}$, in terms of Tikhonov regularization scheme such that

$$
\begin{aligned}
\mathfrak{T}_{\alpha, n r t} & =\sup _{0<\left\|\mathscr{S}^{*} g\right\| \leq 1}\left\langle\mathfrak{R}_{\alpha}^{*} \mathscr{S}^{*} g, u-u_{\infty}\right\rangle, \\
& =\mathfrak{T}_{n r t} \text { for } \quad \alpha \rightarrow 0,
\end{aligned}
$$

which will be shown later.

With the help of last Definition 5.6 we are able to presents one of the key results of this chapter which proves that the range test converges to no response test and vice versa.

Lemma 5.7. The functional of no-response test $\mathfrak{T}_{\alpha, n r t}$ is equal to the functional of range test $\mathfrak{T}_{\alpha, r t}$. If the limits for $\alpha \rightarrow 0$ exist, then the limit functionals are equal as well, i.e. $\mathfrak{T}_{n r t}=\mathfrak{T}_{r t}$

Proof. We start the proof with the definition of $\mathfrak{T}_{\alpha, r t}$ such that

$$
\begin{aligned}
\mathfrak{T}_{\alpha, r t}(G)= & \sup _{\substack{0<\|v\| \leq 1 \\
v \in R\left(\mathscr{S}^{*}\right)}}\left\langle v, \mathfrak{R}_{\alpha}\left(u-u_{\infty}\right)\right\rangle \\
= & \sup _{\substack{0<\|v\| \leq 1 \\
v \in R\left(\mathscr{S}^{*}\right)}}\left\langle\mathfrak{R}_{\alpha}^{*} v, u-u_{\infty}\right\rangle \\
= & \sup _{0<\left\|\mathscr{S}^{*} g\right\| \leq 1}\left\langle\mathfrak{R}_{\alpha}^{*} \mathscr{S}^{*} g, u-u_{\infty}\right\rangle \\
= & \mathfrak{T}_{\alpha, n r t}(G) .
\end{aligned}
$$

After applying the limit $\alpha \rightarrow 0$ and with the help of equation (5.2.14), we are left with

$$
\mathfrak{T}_{r t}(G)=\mathfrak{T}_{n r t}(G)
$$


This completes the proof.

The no response test is based on the following result. For acoustics, the result has been shown in [39]. Here, we will provide a new and different proof, based on transposition.

Theorem 5.8. The flow field $u$ defined in a neighborhood of $\partial \Omega$ is analytically extensible into $\Omega \backslash G$ with boundary values in $W^{1}(\partial G)$ if and only if

$$
\mathfrak{T}_{n r t}(G)<\infty \text {. }
$$

Proof. First, we assume that the flow field is analytically extensible into $\Omega \backslash G$. Then, we can solve the integral equation (5.1.1) in the exterior of $G$ with $\varphi \in L^{2}(\partial G)$ (see Lemma 5.2), such that the measurement values $u_{\text {meas }}$ on $L^{2}(\Lambda)$ are given. We now obtain

$$
\begin{aligned}
\mathfrak{T}_{n r t}(G) & =\sup _{0<\left\|\mathscr{S}^{*} g\right\|_{L^{2}(\partial G)} \leq 1}\left\langle g, u_{\text {meas }}(x)-u_{\infty}\right\rangle \\
& =\sup _{0<\|v\|_{L^{2}(\partial G)} \leq 1}\left\langle\mathscr{S}^{*^{-1}} v, u_{\text {meas }}(x)-u_{\infty}\right\rangle \\
& =\sup _{0<\|v\|_{L^{2}(\partial G)} \leq 1}\langle v, \underbrace{\mathscr{S}^{-1}\left(u_{\text {meas }}(x)-u_{\infty}\right)}_{\varphi}\rangle . \\
& =\sup _{0<\|v\|_{L^{2}(\partial G)} \leq 1}\langle v, \varphi\rangle \\
& <\infty .
\end{aligned}
$$

Now to prove the other direction of the theorem we assume that $\mathfrak{T}_{n r t}(G)<\infty$. Writing

$$
\mathfrak{T}_{\alpha, n r t}(G)=\sup _{0<\left\|\mathscr{S}^{*} g\right\| \leq 1}\left\langle\mathfrak{R}_{\alpha}^{*} \mathscr{S}^{*} g, u-u_{\infty}\right\rangle
$$

where $g \in \operatorname{span}\{\nu\}^{\perp} \subset L^{2}(\Lambda)$ and $\mathscr{S}^{*}$ is an injective operator. From Theorem 5.4 we have

$$
\mathfrak{R}_{\alpha}^{*} \mathscr{S}^{*} g \rightarrow g, \quad \alpha \rightarrow 0 .
$$

Thus applying $\alpha \rightarrow 0$ on equation (5.2.19), we obtain

$$
\begin{aligned}
\lim _{\alpha \rightarrow 0} \mathfrak{T}_{\alpha, n r t}(G) & =\sup _{0<\left\|\mathscr{S}^{*} g\right\| \leq 1}\left\langle g, u-u_{\infty}\right\rangle \\
& =\mathfrak{T}_{n r t}
\end{aligned}
$$

Also, according to equation (5.1.4) we know that the $\lim _{\alpha \rightarrow 0}\left\|R_{\alpha}\left(u-u_{\infty}\right)\right\|<\infty$ if and only if $\left(u-u_{\infty}\right) \in R(\mathscr{S})$. Since we assume that $\mathfrak{T}_{n r t}(G)$ is bounded thus from equation (5.2.19) $\mathfrak{T}_{\alpha, n r t}$ is bounded for all sufficiently small $\alpha$. Now with the help 
of Lemma 5.7 we have $\mathfrak{T}_{\alpha, r t}<\infty$ for all sufficiently small $\alpha$. Thus with the help of Theorem 5.3 we have the analytically extensible velocity field $\left(u-u_{\infty}\right) \in R(\mathscr{S})$ in the exterior of $G$.

With the help of above theorem we can apply the no-response test. We choose several convex test domains $G_{k} k=1, \ldots, n, n \in \mathbb{N}$ and calculate the maximal response $\mathfrak{T}(G)$ for each test domain $G_{k}$ with the help of equation (5.2.4). Now we set a threshold parameter $C$ and check whether the calculated responses for each test domain is bounded by $C$. If we have the domain who's response is bounded by $C$ then with the help of Theorem 5.8 we can say that the field is analytically extensible in the exterior of this particular test domain. The intersection of all those test domains, which have a bounded response by the threshold parameter $C$, such that,

$$
\mathcal{J}:=\bigcap_{k=1}^{n} G_{k},
$$

is a convex set, which is basically a convex approximation of the unknown obstacle $D$.

\subsection{Convergence Test}

In previous chapter we have a detailed discussion on point source method for the reconstruction of the flow field. We calculate the reconstructed velocity field with the help of back projection formula (4.3.19) under the condition (4.3.20). In equation (4.3.19) we calculate the density $g_{\alpha}$ with the help of Tikhonov regularization scheme. For an approximation domain $G$ containing the unknown obstacle $D$ we calculate $u_{\text {rec }}$ for each source point $x \notin G \subset \Omega$. Obviously for inverse problem we do not know about the location of unknown obstacle $D$ and therefore we calculate $u_{\text {rec }}$ for every $x$ in the grid by translating and rotating the approximation domain $G$.

Here an important question arises that where we have a good reconstruction of the flow field and where we have bad reconstruction in the region $\Omega$. For this we need to define an indicator functional $\mathfrak{I}$ which is responsible for identifying the area of interest that is the area which has good approximation. We shall call this area the admissibility region $\mathfrak{D}$. The idea of an indicator function for the admissibility region is given by Erhard in his thesis [10]. He suggested that we can construct a function which tells us in which area we have good field reconstructions. For this we choose two different regularization parameters $\alpha_{1}$ and $\alpha_{2}$ and then with the help of back projection formula (4.3.19), we calculate the reconstructed velocity fields $u_{r e c, \alpha_{1}}$ and $u_{r e c, \alpha_{2}}$, respectively. Now, the indicator function, defined by Erhard,

$$
\Im(x)=\left|u_{r e c, \alpha_{1}}(x)-u_{r e c, \alpha_{2}}(x)\right|, \quad x \in \mathbb{R}^{2},
$$


is the difference of these reconstructed velocity fields. It is observed that this kind of indicator function is a variant of the no response test.

Obviously, for two different regularization parameters $\alpha_{1}, \alpha_{2}$, the reconstructed velocity fields $u_{r e c, \alpha_{1}}, u_{r e c, \alpha_{2}}$, respectively, converges to the true velocity field $u(x)$ as described in equation (4.3.21).

To analyze the convergence in more detail we adopt the Cauchy criterion. Assume that $\left(u_{k}\right)_{k \in \mathbb{N}}$ be a sequence of real numbers. According to the Cauchy criterion we know that

$$
u_{k} \rightarrow u_{0}, \quad k \rightarrow \infty
$$

with some $u_{0} \in \mathbb{R}^{2}$ if and only if

$$
\forall \kappa>0 \exists N \in \mathbb{N} \text { such that }\left|u_{k}-u_{j}\right| \leq \kappa \forall k, j \geq N .
$$

Given a family $\left(u_{k}^{(x)}\right)$ of such sequences, a numerical test for the Cauchy criterion can be obtained by testing the size of $\left|u_{k}^{(x)}-u_{j}^{(x)}\right|$ for some large fixed $k, j \in \mathbb{N}$.

Now we define another indicator function $\mathfrak{T}(x)=\mathfrak{T}_{\kappa, j, k}(x)$ on the family index $x$ by

$$
\mathfrak{T}_{\kappa, j, k}(x):= \begin{cases}1, & \left|u_{k}^{(x)}-u_{j}^{(x)}\right| \leq \kappa \\ 0, & \text { otherwise } .\end{cases}
$$

The true indicator function is given by

$$
\mathfrak{T}_{\text {true }}(x):= \begin{cases}1, & \text { if } u_{k}^{(x)} \rightarrow u_{0}^{(x)}, \quad k \rightarrow \infty \\ 0, & \text { otherwise }\end{cases}
$$

Of course, the convergence test (5.3.4) can only yield an approximation to the true indicator function, since convergence is a statement which is given by the behavior of an infinite number of elements, whereas we only test a finite part of the sequence under consideration.

An alternative test for convergence is obtained by choosing indices $k_{1}, \ldots, k_{m}$ and calculating an approximation to the derivative of $u_{k}$ with respect to $k$ by linear regression of a curve to $u_{k_{1}}, \ldots, u_{k_{m}}$. Fitting a curve of the type

$$
f(t)=a t+b, \quad t \in R
$$

yields an estimate $a^{(x)}=a$ for the slope or derivative of the function. Then, we may define the estimator

$$
\mathfrak{T}_{\kappa, k_{1}, \ldots, k_{m}}(x):= \begin{cases}1, & a^{(x)} \leq \kappa \\ 0, & \text { otherwise }\end{cases}
$$

Thus if our indicator function $\mathfrak{T}_{\kappa}=0$ for certain points then we say that these points belongs to the non convergent region $\mathfrak{D}$. 
In other words we say that if the inequality $\left\|u_{k}^{(x)}-u_{j}^{(x)}\right\| \leq \kappa$ is true then we have the convergence and if not then we deduced that the convergence is not reached.

When we deduced the non-convergent regions with the help of indicator function then the intersection of all these non-convergent region is an approximation of our unknown domain $D$, i.e.,

$$
D=\bigcap_{j} \mathfrak{D}_{j} \quad j \in \mathbb{N}
$$

We observe that the convergence test is a variant of no-response test. In convergence test we calculate two different densities $g_{\alpha_{1}}$ and $g_{\alpha_{2}}$ for two different reconstructed velocity fields $u_{r e c_{1}}, u_{r e c_{2}}$ for two different regularization parameters $\alpha_{1}$ and $\alpha_{2}$ and then apply the Cauchy criterion on them. Now with these two densities we can define a new density which is the difference of these two densities. Taking the difference of these reconstructed velocity fields, we have

$$
\Im(x)=\left|u_{\alpha_{1}}(x)-u_{\alpha_{2}}(x)\right| \leq\|\int_{\Lambda} \underbrace{\left(g_{\alpha_{1}}^{T}(y)-g_{\alpha_{2}}^{T}(y)\right)}_{:=g^{T}(y)}\left(u_{\text {meas }}(y)-u_{\infty}\right) d s(y)\|_{L^{2}(\partial G)} .
$$

Thus we have

$$
\mathfrak{I}(x) \leq\left\|\int_{\Lambda} g^{T}(y)\left(u_{\text {meas }}-u_{\infty}\right) d s(y)\right\|:=\mathfrak{r}_{1}(g) .
$$

with

$$
\left\|\int_{\Lambda} g^{T}(y) E(y-\cdot) d s(y)\right\| \leq 1
$$

Now if we compare the last equation with (5.2.1), we readily see that this is a response $\mathfrak{r}(g)$ on $\partial G$. With this response we can observe the analytical extension of the velocity field as describe in section 5.2. This provides the relation of convergence test with the no response test.

Theorem 5.9. Consider an arbitrary admissible domain $B \subset \Omega$ which contains our approximation domain $G$. Assume that the point source method converges uniformly with respect to the maximum norm on $\partial B$ then $u_{\text {meas }}$ analytically extensible upto $\mathbb{R}^{2} \backslash B$.

Proof. Assume that the point source method converges uniformly on $\partial B$. This means that with $g_{\alpha}$ given by

$$
g_{x, \alpha}=\mathcal{R}_{\alpha}^{*} E(x-\cdot)
$$

the reconstructed density

$$
u_{\alpha}(x)=\int_{\Lambda} g_{x, \alpha}^{T}\left(u_{\text {meas }}-u_{\infty}\right) d s
$$


converges towards some velocity field $u_{*}$. Using the definition

$$
\varphi_{\alpha}:=\mathcal{R}_{\alpha}\left(u_{\text {meas }}-u_{\infty}\right)
$$

we now employ the duality (5.3.9) to obtain

$$
\begin{aligned}
u_{\alpha}(x) & =\left\langle g_{\alpha}, u_{\text {meas }}-u_{\infty}\right\rangle \\
& =\left\langle\mathfrak{R}_{\alpha}^{*} E(x-\cdot), u_{\text {meas }}-u_{\infty}\right\rangle \\
& =\left\langle E(x-\cdot), \mathfrak{R}_{\alpha}\left(u_{\text {meas }}-u_{\infty}\right)\right\rangle \\
& =\mathscr{S} \varphi_{\alpha}(x) \rightarrow u_{*}(x) \text { for all } x \in \partial B \text { and } \alpha \rightarrow 0 .
\end{aligned}
$$

This means that the single-layer potential $S \varphi_{\alpha}$ converges to $u_{*}$ on $\partial B$ and by construction it converges towards $u_{\text {meas }}-u_{\infty}$ on $\Lambda$. Thus, the limit is a solution to the Oseen equation in $\Omega \backslash \bar{B}$ with values $u_{\text {meas }}-u_{\infty}$ on $\Lambda$, i.e. $u_{*}+u_{\infty}$ is an analytic continuation of the given measured values $u_{\text {meas }}$ on $\Lambda$, and the proof is complete. 


\section{Part II}

\section{Numerics}





\section{Chapter 6}

\section{Domain Sampling}

There are several numerical algorithms available in the literature [38] to solve the inverse scattering problems, for example the Needle approach, Domain Sampling or the LASSO scheme. We extend these techniques to the inverse fluid dynamics. Although the Needle approach is a nice theoretical tool, however for the convergence of the method this approach is highly unstable because of the strong non-convexity of the corresponding approximation domain $G$. Due to this disadvantage we use domain sampling and LASSO scheme in this monograph, which are quite reasonable in the sense of stability although these algorithms are time consuming. The goal of this chapter is the numerical realization of the point source method and the convergence test with the help of domain sampling technique.

\subsection{Direct Problem}

In this monograph, we parametrized all our domains over the interval $[0,2 \pi]$ and employ the algorithms based on a uniform mesh defined by

$$
t_{j}=\frac{2 \pi j}{N}, \quad j=0, \ldots, N-1, \quad N \in \mathbb{N} .
$$

In two dimensions, generally, the parametrized form of the boundary $\partial G$ of any domain $G$ can be written as

$$
z(t):=\left(p_{1}(t), p_{2}(t)\right), \quad t \in[0,2 \pi]
$$

with appropriate trigonometric polynomials $p_{1}$ and $p_{2}$. For numerical calculations we use the boundary points

$$
x_{j}=z\left(t_{j}\right), \quad j=1, \ldots, N-1 .
$$




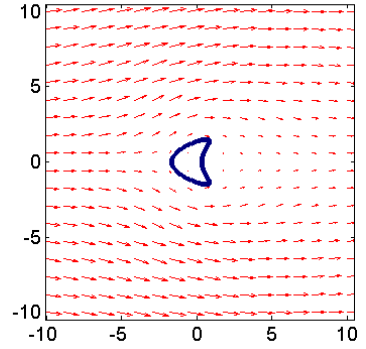

(a)

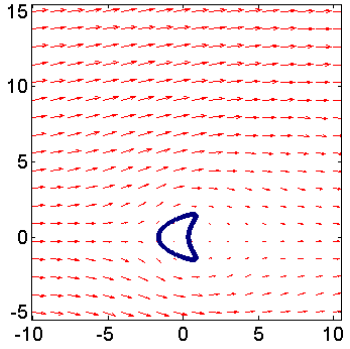

(b)

Figure 6.1: Figure 6.1(a) explains the behavior of flow field near the boundary and the figure 6.1 (b) shows that the velocity field is constant at infinity i.e., $u_{\infty}=(1,0)$

As described in Definition 2.27 that the integral of a function $\mathcal{F}$ on $\partial G$ can be approximated by some quadrature rule, i.e.,

$$
\int_{\partial B} \mathcal{F}(y) d s(y) \approx \sum_{j=0}^{N-1} w_{j} \mathcal{F}\left(x_{j}\right), \quad j=0, \ldots, N-1
$$

where $w_{j}$ are the quadrature weights. There are several methods available in literature to deal with the integral equations, here we use the Nyström type method. In Nyström's method we discretized the integral equation of second kind by using a quadrature rule as described above. A short theoretical description of the Nyström's method is given in section 2.5.

For the direct problem we use simulated measurements $u_{\text {meas }}$ on the surface $\Lambda \subset \partial B$. To calculate $u_{\text {meas }}$ we employ a Nyström's type method applied to the integral equation (3.2.31). The discretized version of equation (3.2.31) is given by

$$
\sum_{j=1}^{n} E\left(x_{k}-y_{j}\right) \varphi\left(y_{j}\right) s_{j}+\operatorname{grad} \Phi\left(x_{k}\right) \sum_{j=1}^{n} \varphi\left(y_{j}\right) \cdot \nu\left(y_{j}\right) s_{j}=f\left(x_{k}\right)-u_{\infty}, k=1,2, \ldots n
$$

where $s_{j}$ are the quadrature weights. We parametrized the boundary $\partial D$ on the interval $[0,2 \pi]$ such that $\partial D=\{z(t): 0 \leq t \leq 2 \pi\}$ denote a differentiable and $2 \pi$ periodic parametrization with a regular grid on $[0,2 \pi)$ and $x_{k}=z\left(t_{k}\right)$ for $k=1, \ldots, n$ for $n \in \mathbb{N}$. Writing $\varphi_{j}=\varphi\left(y_{j}\right)$ and using $\varphi=\left(\varphi_{j}\right)_{j=1, \ldots, N}$ this leads to a matrix equation of the form

$$
\mathbf{A} \varphi=\mathbf{f}-\mathbf{u}_{\infty}
$$




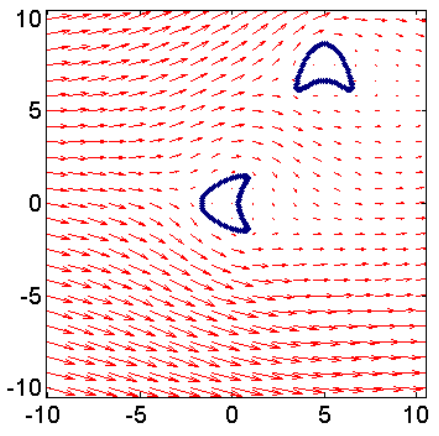

(a)

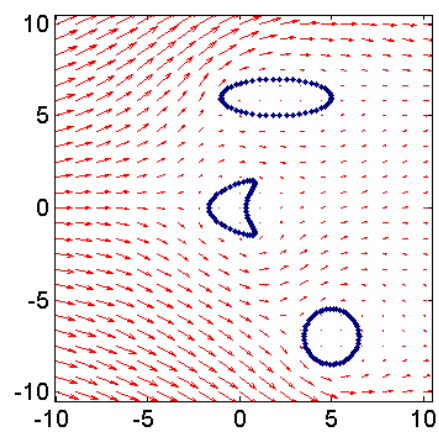

(b)

Figure 6.2: Flow field for the Oseen equation with more than one obstacle

in $\mathbb{R}^{2 n}$, where

$$
\mathbf{A}=\left\{A_{i j}\right\}=(\underbrace{E\left(x_{i}-y_{j}\right)}_{2 n \times 2 n} s_{j}+\underbrace{\operatorname{grad} \Phi\left(x_{i}\right)}_{2 n \times 1} \underbrace{\nu^{T}\left(y_{j}\right)}_{1 \times 2 n} s_{j})_{\substack{i=1,2, \ldots, n \\ j=1,2, \ldots, n}} .
$$

Since in the modeling of Oseen equation, we assumed that the flow is coming from the left side so we take the constant velocity field $u_{\infty}=(1,0)$. Thus with the help of standard tools we calculate the unknown density $\varphi$ from equation (6.1.1). Once we have the vector $\varphi$, then, the discretized version of (3.2.32) provides an approximation to the solution of the Oseen equation in the exterior of $D$ i.e., on $B$ and on $\Lambda$. We use a discretization of $z_{1}, \ldots, z_{m}$ of $\Lambda$ and calculate the velocity field, $u_{\text {meas }}\left(z_{j}\right)$, at $\Lambda$ for $j=1, \ldots, m$, leading to a vector $u_{\text {meas }} \in \mathbb{R}^{2 m}$.

Algorithm 6.1. The algorithm of the direct problem for the Oseen equation is as follow:

1: Choose a domain $D$ such that $0 \in D$

2: Parametrize the boundary $\partial D$ on the interval $[0,2 \pi]$

3: Discretize the integral equation (3.2.31) with the help of Nyström method

4: Calculate the unknown density $\varphi$ from equation (6.1.1)

5: Calculate the velocity field $u$ by substituting back the density $\varphi$ in the discretized version of the pair (3.2.32)

Examples: With the help of following examples we demonstrate the simulation of the direct problem. We analyzed the behavior of the fluid near the boundary of the obstacle and away from the obstacle. 
In our first example, we choose the well known kite shaped domain $\mathrm{D}$, whose parametric representation is given by

$$
x(t)=(\cos t+0.65 \cos 2 t-0.65,1.5 \sin t), \quad 0 \leq t \leq 2 \pi .
$$

For this and next experiment, for the direct computation, we used 150 sampling points for $\partial D$. We did our computation on two different grids for the better understanding of the flow around the obstacle and its behavior far from the obstacle (see Figure 6.1). Figure 6.1(a) exhibits the paraboloid wake region behind the obstacle, as suggested by Oseen [33], as well as the flow behavior at the stagnation points. While figure 6.1(b) tells us that the flow field is constant at infinity.

In the second experiment (see Figure 6.2(a)) we used two kite shaped obstacle keeping origin inside one of them. Here the discretization points for the boundary of each obstacle are equally divided i.e., 75 each. In the last we did our simulation with three obstacles, an elliptic, a circular and a kite shaped obstacle. The parametric representation of circular and elliptic obstacles are given by

$$
\begin{aligned}
& z(t)=(3 \cos t, \sin t+7), \\
& z(t)=(\cos t, \sin t),
\end{aligned}
$$

respectively, here $t \in[0,2 \pi)$. This experiment clearly demonstrate the attitude of flow field around the obstacles. Figure 6.2(b) tells us that the algorithm for the direct problem works for more than two obstacles as well. Now with this basic knowledge of direct problem we proceeds ourselves for the inverse problem.

\subsection{Point Source Method}

In this section we will illustrate the point source method for the flow field reconstruction with some numerical examples. As in the Chapter 4 we observed that the point source method is applicable only under the condition that our unknown domain $D$ must lie inside the approximation domain and the source point $x$ lies in the exterior of $G$, i.e., $\mathbb{R}^{2} \backslash G$. For this reason the choice of the sampling technique is an important issue.

In inverse scattering theory, several techniques are available for the reconstruction of unknown domains and the approximation of a scattered field, for example the point sampling, the needle approach proposed by Ikehata [19,20], or the domain sampling proposed by $[37,41]$. Here we give a brief review of these sampling techniques.

Among all of them, the most simplest sampling scheme is point sampling which is widely used for the different versions of factorization method. In this technique we choose a point $z \in \mathbb{R}^{m}$ and with an appropriate scheme we check whether this point belongs to the interior of the unknown domain $D$ or not. However this technique is 
not possible in our work since the approximation of the solution of Oseen equation is only possible on set $G$ where the solution is regular.

Theoretically, the most strong technique is needle approach which ensures the unknown domain $D$ is inside the approximation domain $G$. A continuous curve $r$ is called a needle if it maps from $r:[0,1] \rightarrow \Xi$ with $r(0), r(1) \in \Xi$ for $0<t<1$. Also we assume another domain $B$ to large enough to contain the unknown domain $D$. In a most simple case we take a straight line whose one end is outside the domain $B$ and the other end belongs to $B$. Then the approximation domain $G$ is chosen as a subset of $B$ and the complement of this curve (needle). Thus, we can use these curves (needles) to probe the area under consideration for the unknown domain $D$. However numerically the needle approach is unstable because the approximation domain $G$ is highly non convex. For a more detail study we refer the reader to $[19,20,38]$.

The domain sampling technique is quite stable technique numerically as well as theoretically. The idea of the domain sampling technique is to choose an approximation domain $G$ and test whether the desired quantity is inside this test domain $G$ or not. If the desired quantity is inside then we call it a positive test domain. We carry out this procedure for several test domains and then obtained the desired quantity by taking the intersection of all positive test domains. Or in other way around we can say that the domain sampling is a technique in which we carry out the reconstruction of velocity field $u_{r e c}$ for many different test domains $G$ and test the convergence on the boundary $\partial G$ and the exterior of these domains. With the help of masking operation we can combine all good reconstructions of velocity field

$u_{\text {rec }}$. The exterior of this reconstruction field inside the flow region is basically our unknown obstacle $D$. The convergence of the domain sampling is shown in [41].

Among these techniques we focus on domain sampling scheme which is quite reasonable for the reconstruction of the flow field as well as to find out the location of the unknown obstacle.

\section{Choice of Approximation Domain}

The choice of approximation domain is a big milestone in domain sampling technique when we apply the point source method for the inverse fluid flow problems. In his first article about point source method [34], Potthast describes how to choose the approximation domain. With the passage of time there are some other techniques developed for the choice of the approximation domain as well. In our analysis we adopt the classical way for the choice of approximation domain as described in, for example, [10,34,38].

In the point source method it is the primary requirement that the source point $x$ must be in the exterior of the approximation domain $G$. For the choice of the approximation domain we first choose a fixed reference domain $G_{0}$ such that $0 \notin$ $G_{0}$. Then with the following setting we construct the approximation domain $G$ via 
translation and rotation given by

$$
G_{x}=G(x)=\left\{M z+x, z \in G_{0}\right\}
$$

for $M \in O S(m, \mathbb{R})$ and $x \in \Omega$. Here $O S(m, \mathbb{R})$ is the set of real and orthogonal $m \times m$ matrices with

$$
\operatorname{det}(M)=1 .
$$

This particular setting first rotate the fixed approximation domain and then translate it for every point $x$ in the domain. Figure 6.3 exhibits the setting of our reference domain $G_{0}$. Since $0 \notin G_{0}$ we observed that all approximation domains fulfill the condition $x \notin G(x)$ for each source point $x \in \Omega \backslash G$.

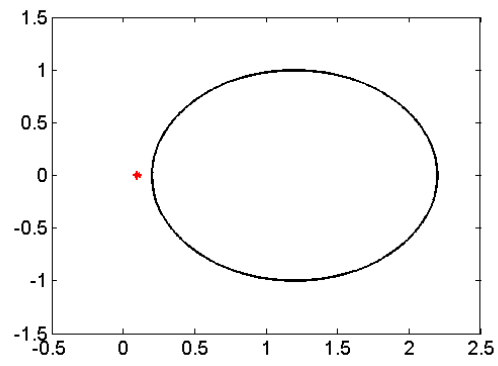

(a)

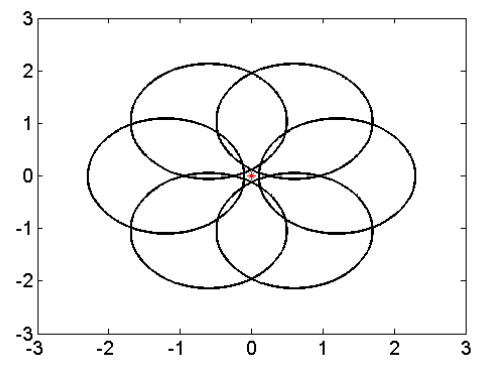

(b)

Figure 6.3: Left figure shows how we choose our source point around the approximation domain, while the right figure represents the rotation of approximation domain around the source point.

\section{Masking Factor}

As it is discussed before that in our algorithm we translate and rotate the approximation domain around a source point $x$. In numerical calculation the rotation argument plays an important role in the reconstruction of the flow field. Assume that we rotate our approximation domain in $L$ different directions around the source point, as shown in Figure 6.3(a), then for each direction we have reconstructed velocity field $u_{r e c}$ for that particular source point. To get best reconstruction among them we use the following masking technique.

In the first step we employ the Tikhonov regularization scheme to calculate the densities

$$
g_{x, \alpha}=\left(g_{x, 1, \alpha}, g_{x, 2, \alpha}\right)
$$

with regularization parameter $\alpha>0$ which satisfy (4.3.20) by

$$
\left(\alpha I+S S^{*}\right) g_{x, j, \alpha}=S E_{j}(x-.), \quad j=1,2
$$


Discretization is carried out via Nyström's method as described above. Then, we use the discretized version of the back projection formula (4.3.19) with two different Tikhonov regularization parameters $\alpha_{1}$ and $\alpha_{2}$ to calculate the reconstructed velocity fields $u_{r e c, \alpha_{1}}$ and $u_{r e c, \alpha_{2}}$. In the next step we calculate the discretized version of indicator function defined in equation (5.3.1). Now we define a threshold parameter $\kappa>0$ and with the help of indicator function we try to locate those points where the difference of these reconstructed velocity fields are small such that

$$
\text { mask }:= \begin{cases}1, & \left|u_{r e c, \alpha_{1}}-u_{r e c, \alpha_{2}}\right| \leq \kappa \\ 0, & \text { otherwise } .\end{cases}
$$

Obviously the functional mask approaches zero for those source points $x$ where we have do not have good reconstruction of the velocity field $u_{r e c}$. Now in the last step of the masking operation we obtain the final reconstruction of the flow field by multiplying the mask obtained in equation (6.2.3) with the component of the calculated velocity field such that,

$$
u_{\text {rec }_{i}}=\left(\sum_{k=1}^{L} \operatorname{mask}(k,:) u_{\text {rec }_{i}}\right) \frac{1}{\max \left(1, \sum_{k=1}^{L} \operatorname{mask}(k,:)\right)} \quad i=1,2 .
$$

Thus, in this way we have a good reconstruction of flow field $u_{r e c}=\left(u_{r e c_{1}}, u_{r e c_{2}}\right)$.

With this final tool we are able to summarize the following algorithm for the point source method.

Algorithm 6.2. For the inverse fluid flow problems, the point source method calculates an approximation $u_{r e c}$ of the true velocity field $u$ on a grid $\mathcal{G}$ by the following steps:

1: Choose a fixed reference domain $\left(0, G_{0}\right)$ such that $0 \notin G_{0}$.

2: For some rotation $M=M_{\ell}$ and each $z$ in the grid $\mathcal{G}$ calculate a rotated and translated approximation domain $G(z)$ by (6.2.1).

3: Calculate $g_{z, \alpha}$ via (6.2.2) with a regularization parameter $\alpha=\alpha_{1}$.

4: Calculate $u_{r e c, \alpha}$ by the PSM reconstruction formula (4.3.19).

5: Repeat step $3-4$ for another Tikhonov regularization parameter $\alpha=\alpha_{2}$.

6: Calculate mask with the help of (6.2.3).

7: Repeat step $2-5$ for $L$ different rotations $M_{\ell}, \ell=1, \ldots, L$ of the approximation domain.

8: Finally, calculate $u_{r e c}$ via (6.2.4).

Remark: The point source method is designed for the reconstruction of the flow field as well as for the shape of the unknown obstacles by assuming that our reconstructed velocity field is small near the boundary. Using this property we can locate the unknown obstacle in the flow field. We would like to mention that there is no restriction on the number of unknown obstacles for reconstruction process. However, it is observed that the error level increases with increasing the number of obstacles. 
Remark: (Effect of Viscosity) Viscosity is a terminology for measuring the thickness of the fluid. As in this monograph our major focus is on Oseen equation which was derived on the assumption that the viscous forces are much stronger then the inertia forces. In principle due to this fact we are bound to take those fluids which are highly viscous (See details in Section A) but off course we can not neglect the inertia forces as well since our governing equation represents the Oseen Flow.
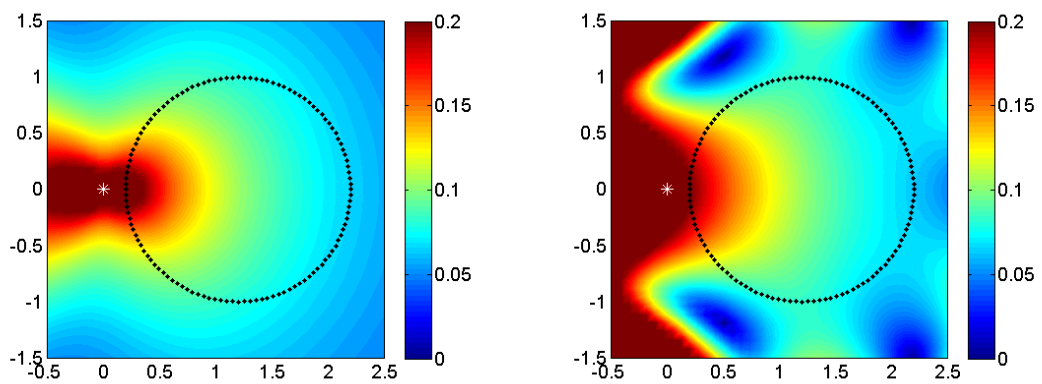

Figure 6.4: Fundamental Solution $E(0-\cdot)$ (left) and its approximation (right) for the circular approximation domain $G_{0}$

More precisely, the fundamental solution of the Oseen flow (3.1.32) tells us that we must have a bound on the viscosity parameter, i.e., we can not take viscosity parameter too large since our fundamental solution approaches to zero and because of this we have very bad results. On the other hand we are not allowed to take small viscosity parameter $\mu$ due to the nature of fluid. We did a number of experiments to see the lower and upper bound of viscosity parameter for an inverse problem. We observe that for a good field reconstruction we can take the viscosity parameter $\mu=1$ to $\mu=20$.

In this monograph we only presents the reconstruction algorithm when a maximum of two unknown obstacles are involved. First we present our results for the field and shape reconstruction with one unknown obstacle.

\section{Field reconstruction with one obstacle}

Before presenting some examples we want to check our key result, numerically, that whether the approximation of the point source $E(x-\cdot)$ with the single layer potential operator $S^{(a d j)} g$ given in equation (4.3.20) is good enough inside the approximation domain or not. Since for the appropriate choice of the domains the operator $S^{(a d j)}$ is equal to the adjoint operator $S^{*}$ of single layer potential operator $S$ which is defined in equation (4.1.2). We use Nyström's method to calculate an approximation of the fundamental solution $E(0-\cdot)$ on $G_{0}$ by solving the following regularized integral equation

$$
\left(\alpha I+S S^{*}\right) g_{0}=S E(0-\cdot)
$$


on $\left(L^{2}\left(\partial G_{0}\right)\right)^{2 \times 2}$ for $g_{0}$. We used Tikhonov regularization scheme to solve the illposed behavior of the problem.

We solve the equation $(6.2 .5)$ by separating the fundamental solution $E(0-\cdot)$ in to two parts, i.e.,

$$
\begin{aligned}
& \left(\alpha I+S S^{*}\right) g_{0,1}=S E_{1}(0-\cdot) \\
& \left(\alpha I+S S^{*}\right) g_{0,2}=S E_{2}(0-\cdot)
\end{aligned}
$$
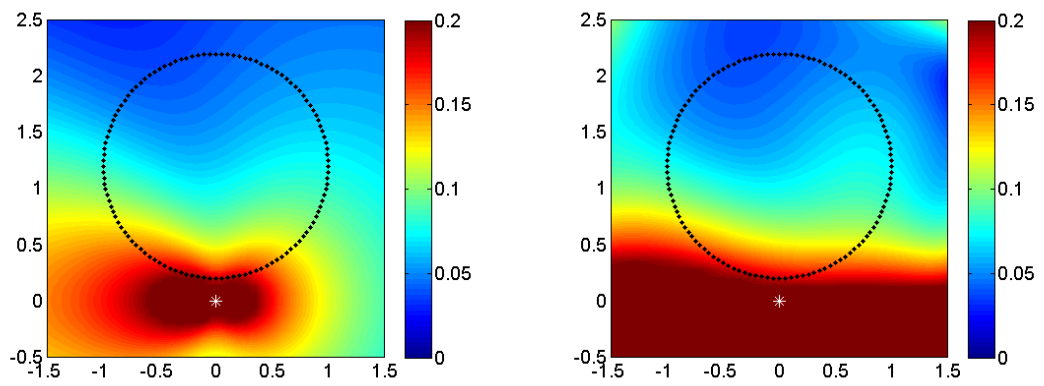

Figure 6.5: Fundamental Solution $E(0-\cdot)$ (left) and its approximation (right) for the circular approximation domain $G_{0}$

In Figure 6.4 we did our computation on $[-0.5,2.5] \times[-1.5,1,5]$, while the value of the regularization parameter $\alpha$ is $10^{-10}$. We set the circular approximation domain with a radius $r=1$. To obey the restriction $0 \notin G_{0}$, we took the center of the circle at $\left(r+\epsilon_{r}, 0\right)$ such that it is in distance $\epsilon_{r}=0.2$ of the source point $x=0$. We discretized the boundary of the approximation domain with 120 sampling points, while we choose the viscosity parameter $\mu$ is 1 .

In Figure 6.5 we took a different setting of the boundary of approximation domain $G_{0}$ relative to the source point $x=0$ for the better understanding of the quality of approximation inside the approximation domain. Here one can see that we have a very nice approximation of the fundamental solution $E(x-\cdot)$ with the single layer potential inside the approximation domain $G_{0}$. Figure 6.6 shows us the difference between the point source $E(0-\cdot)$ and its approximation $v$ with different settings for the color bar. It is observed that the quality of approximation is best in the center of the approximation domain $G_{0}$.

Examples: As a first example we choose the famous kite shaped obstacle as an unknown obstacle $D$ whose parametric representation is given in equation (6.1.2). In the first step of the algorithm 6.2 we have to choose an approximation domain $G_{0}$ such that the origin must belongs to the exterior of $G_{0}$. As our assumption of the inverse problem is that we do not have any knowledge of the shape or the location of the obstacle $D$, it is better to choose a large and smooth shaped circular 
approximation domain $G_{0}$ such that $0 \notin G_{0}$ with radius for example $r=3$. We discretized the boundary of the approximation domain and the measurement boundary with 140 and 200 points respectively. We did our experiment on $[-4,4] \times[-4,4]$ with $20 \times 20$ grid points. In this particular experiment, we choose the value of the viscosity parameter $\mu$ as 5 .
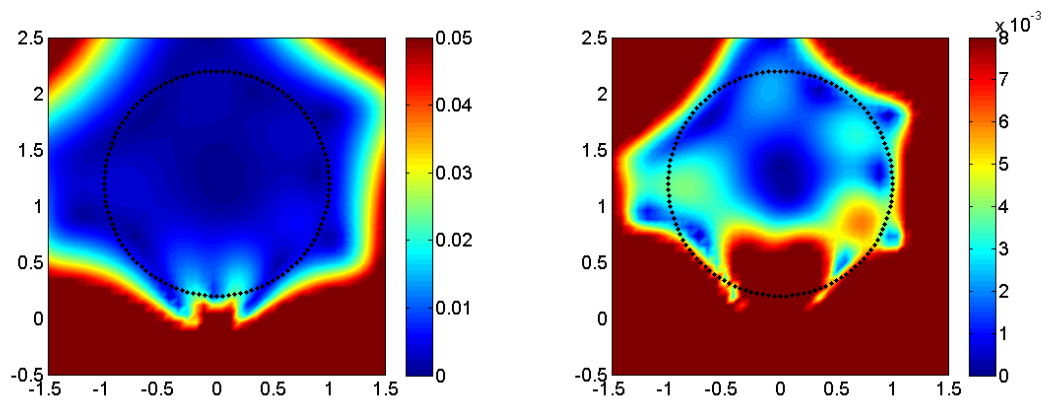

Figure 6.6: Difference between the approximation $v$ and the point source $E(0-\cdot)$ for different settings of the color bar

Now as a second step of the algorithm 6.2 we want to establish an approximation domain $G$ for each source point $x \notin G_{x}$ in the grid. As described in equation (6.2.1), we first translate the fixed approximation domain $G_{0}$ for each source point $x$ and then we implement the rotation around each source point $x$. For rotating the approximation domain we used the following rotation matrix

$$
M=\left(\begin{array}{cc}
\cos \theta & -\sin \theta \\
\sin \theta & \cos \theta
\end{array}\right) .
$$

with $\theta=2 \pi / L$ where $L \in \mathbb{N}$ is a rotating parameter and it determines how many time we rotate our approximation domain around the source point. In this example we take $L=6$ for rotation as shown in Figure 6.7

After setting of the approximation domain for each source point $x$ we calculate the density $g_{x}$ in the same way as we calculated the density $g_{0}$ with the help of equations (6.2.5)-(6.2.7). For this we again use the Nyström's method to calculate an approximation of the fundamental solution $E(x-\cdot)$ on $G_{x}$ by solving the following regularized integral equation

$$
\left(\alpha I+S^{*} S\right) g_{x}=S^{*} E(x-\cdot)
$$

on $L^{2}\left(\partial G_{x}\right)$ for $g_{x}$. Here the value of the Tikhonov regularization parameter is $10^{-9}$. Once we have the density $g_{x}$ for each source point $x$, then with the help of the back projection formula (4.3.19), we calculate the reconstructed velocity field $u_{\text {rec }}$ for each source point $x$. Since it is discussed in the before that due to the rotation matrix we have several reconstructed velocity fields for a particular source point. 

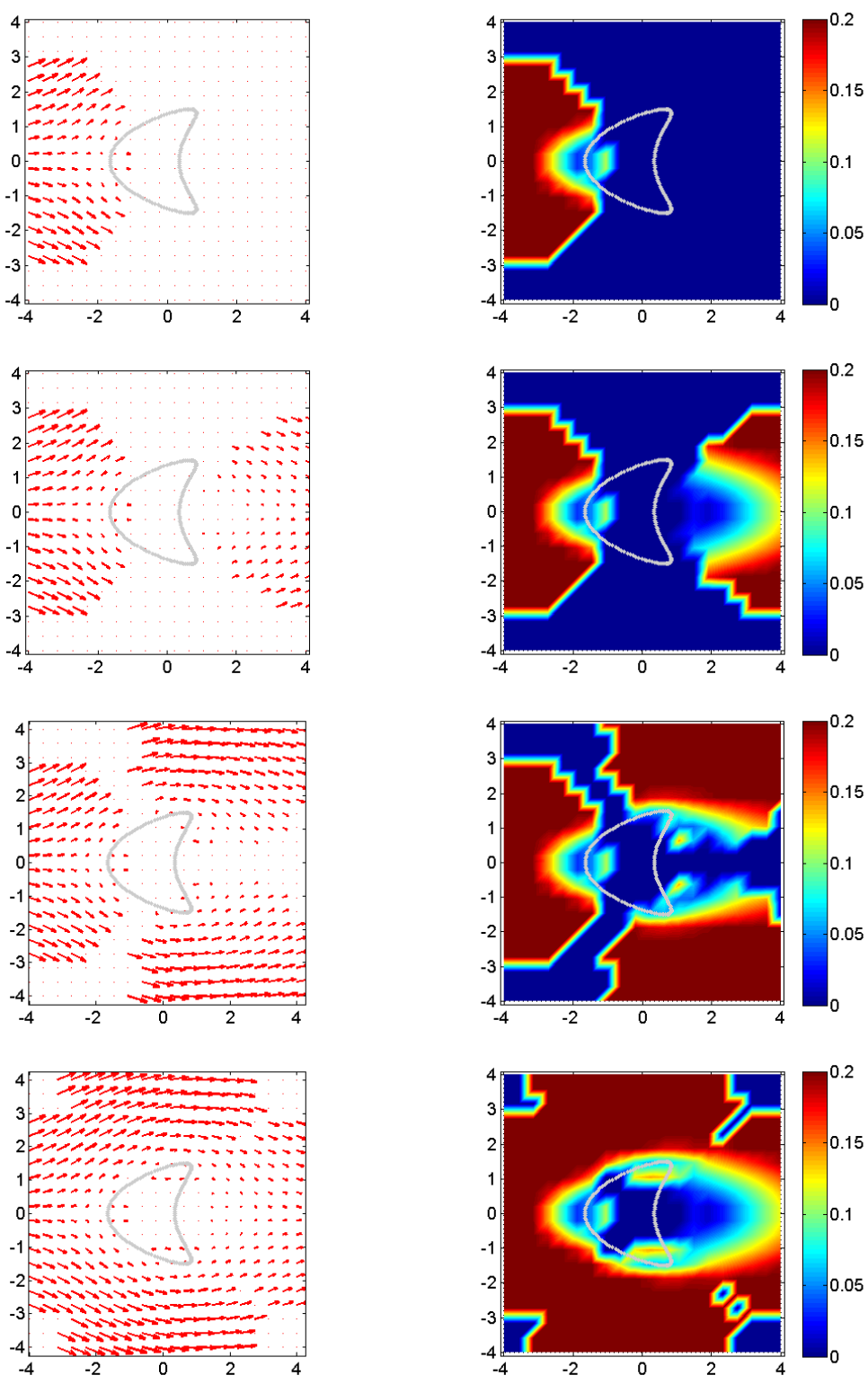

Figure 6.7: We show the effect of domain sampling according to equation 6.2.4, which leads to reconstructions on different parts of the space. We choose $L=1, \ldots, 4$ in the four rows, respectively, leading to valid reconstructions in more and more points. Dark blue represents zero which is given when no valid reconstruction has been achieved. 
Now using the masking technique as explained in equations (6.2.3) and (6.2.4), we calculate the final reconstruction of the velocity field. We take the value of cut off parameter $\kappa=0.015$ to construct the mask functional.

In Figure 6.7 we exhibit the role of rotation parameter $L$. The first row represents the reconstruction when we choose the rotating parameter $L$ equal to one, or in other words we can say that we only translate the approximation domain by setting the source point on its left. In the second row we take $L=2$, this means that our approximation domain rotates around the angel $\theta=0, \pi$. Similarly in the fourth row of the Figure 6.7 we rotate the approximation domain around the angle $\theta=0, p i / 2, \pi, 2 \pi$, on the right side of the figure we can see that on every corner we do not have good reconstruction. From this analysis we observe that with the large number of rotation parameter $L$ we have good reconstruction of the whole flow field.
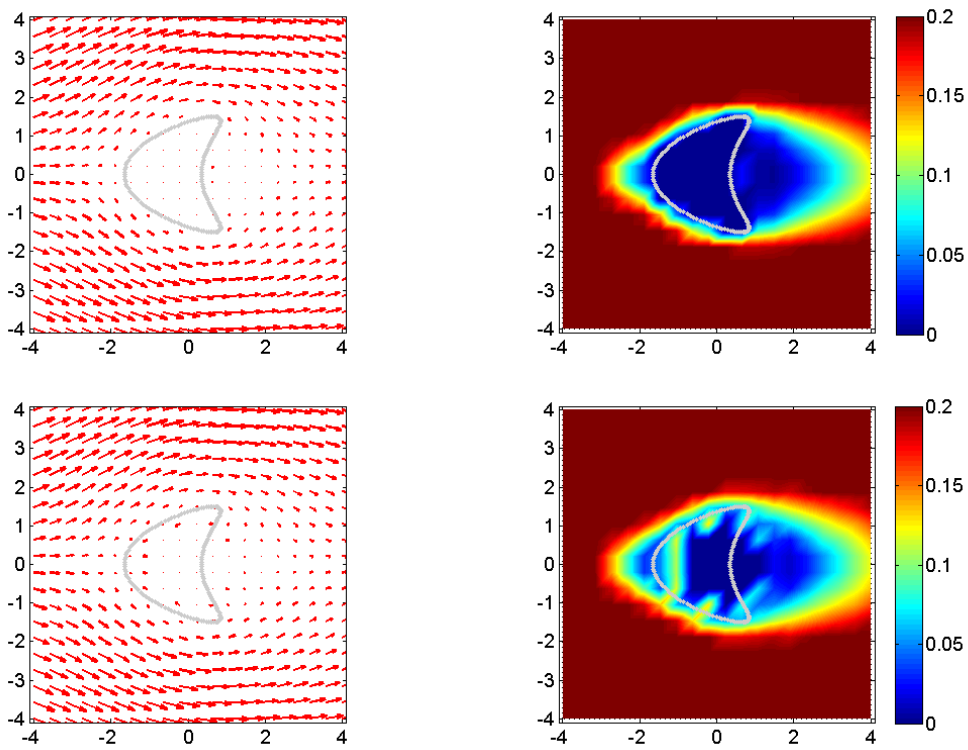

Figure 6.8: First row shows the original flow field around a kite shaped obstacle, while in the second row the reconstruction is presented. Note that for the reconstruction the unknown inclusion (white curve) is not known.

In Figure 6.8 we analyzed that taking $L=6$ gives us the full reconstruction of the flow field in this particular example. We can see that we have very good results behind the obstacle i.e., in the wake region, as well as in front of the obstacle i.e., on stagnation points. To observe the reconstruction in the wake region behind the obstacle in more detail we used our algorithm in the grid of $20 \times 15$ points on the frame of $[-6,15] \times[-6,6]$. We use an elliptic obstacle whose parametric representation is

$$
x(t)=(\cos t, 2 \sin t), \quad 0 \leq t \leq 2 \pi .
$$


We applied the point source method with same data as we used in the first example. In the end we obtained a parboiled reconstructed wake region behind the obstacle and after some particular distance the flow behaves normally (See Figure 6.9).

\section{Field reconstruction with two obstacles}

Now we implement the point source method to reconstruct the flow field with two obstacles. We divide our inverse problem into two problems, i.e., first we reconstruct the which we have the knowledge of the obstacle and we have to reconstruct the flow field around those obstacle. From physical point of view these problems exists for example when we want to reconstruct the flow around two submarines or we have a good knowledge of the bridge and we want to observe the behavior of the flow around its two pilers.
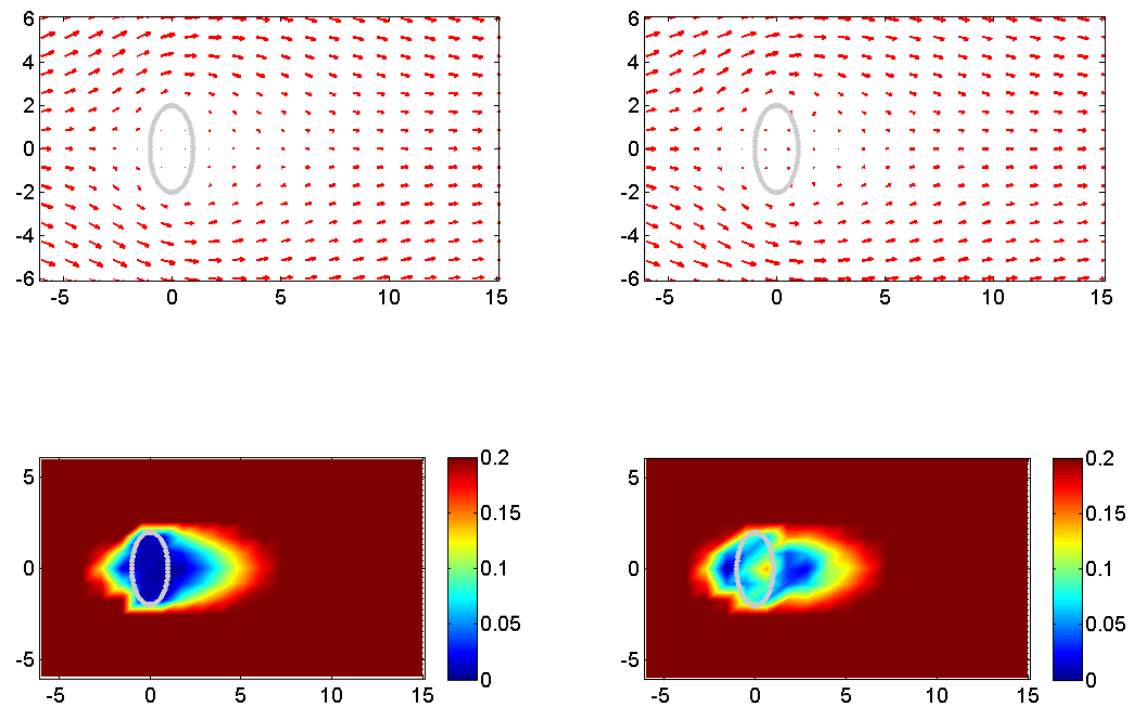

Figure 6.9: This figure exhibit the flow visualization behind the obstacle when the flow is far behind the obstacle. The left column represents the original flow field while the reconstruction is shown in right column. Here, the inclusion is shown as gray curve, which is unknown to the reconstruction algorithm.

We have two options for the choice of approximation domain either we can take the approximation domain as a single unit or we can split it into two sub domains according to the known obstacles. We did our analysis with both options of the approximation domain.

First we split the approximation domain into two circular shaped sub domains 


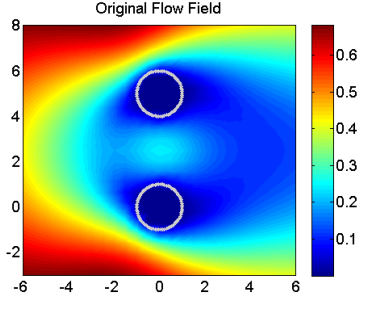

Original Flow Field

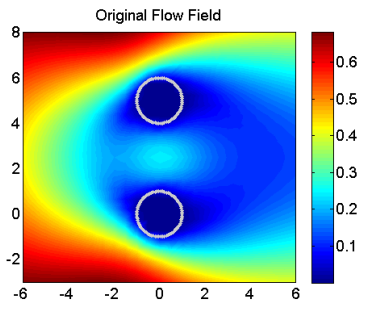

Original Flow Field

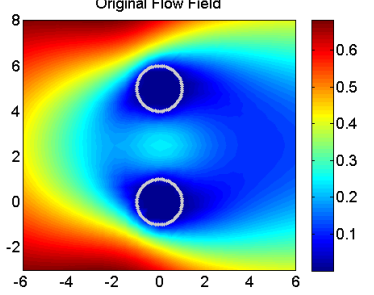

Original Flow Field

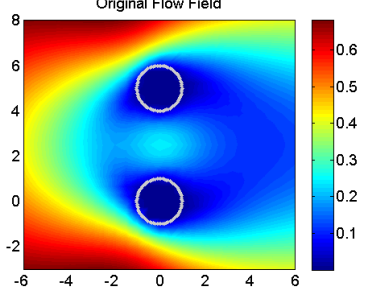

Original Flow Field

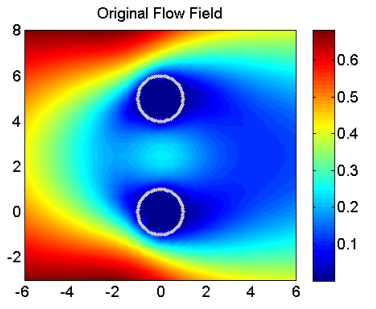

Reconstruction, $r=1$

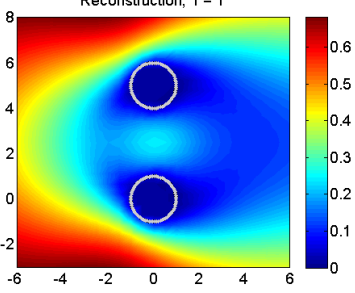

Reconstruction, $r=1.7$

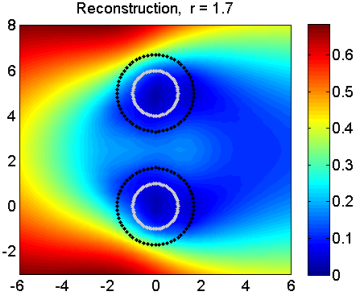

Reconstruction, $r=2.2$
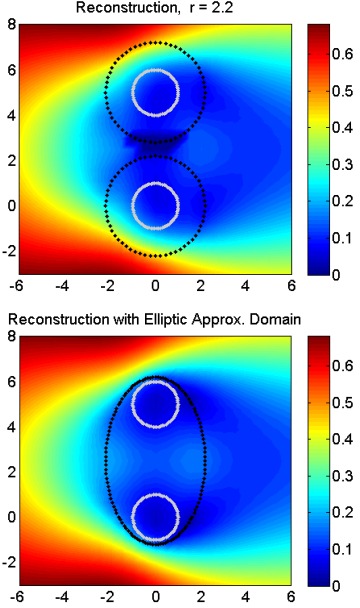

Reconstruction with Elliptic Approx. Domain

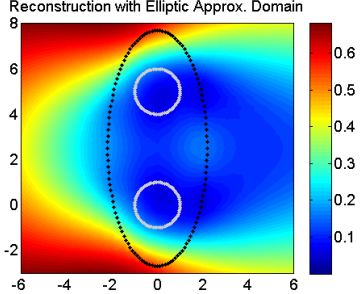

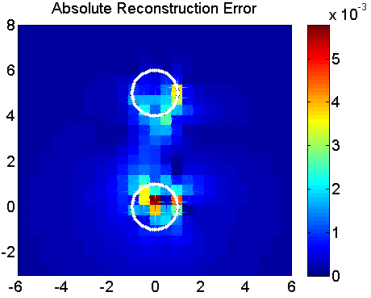

Absolute Reconstruction Error

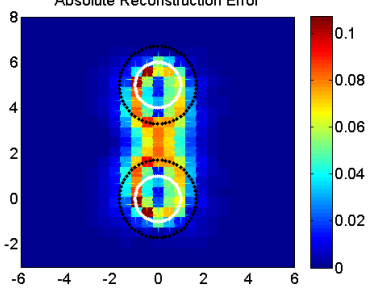

Absolute Reconstruction Error

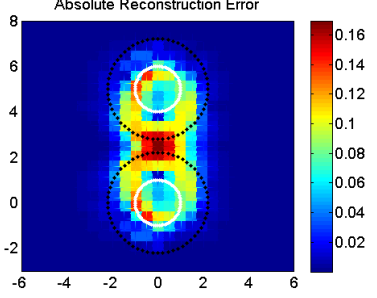

Absolute Reconstruction Error

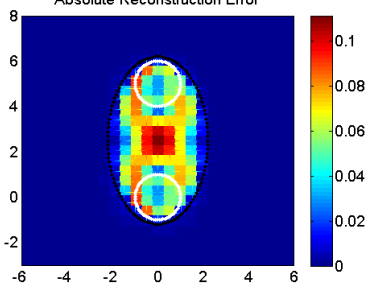

Absolute Reconstruction Error

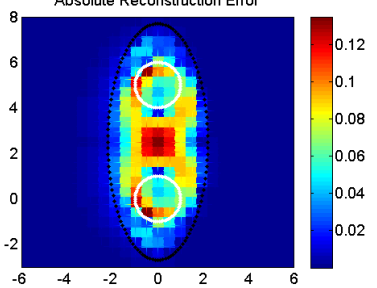

Figure 6.10: First column exhibits the original flow field with the two obstacles. Second column represents the reconstructed flow field. While in the third column we present the absolute reconstruction error. The first three lines shows that our error level increases with the increase of the size of approximation domain $G$. From last two lines we analyzed that if we take an ellipse shape approximation domain $G$ which contains both the unknown obstacles then we do not have good reconstruction in the area between the unknown obstacles. This kind of comparison tells us that if we take the fixed circular approximation domain which is approximately equal to original size of the obstacles then we can reduce the error level. 


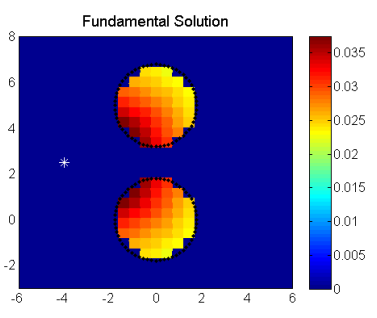

Fundamental Solution

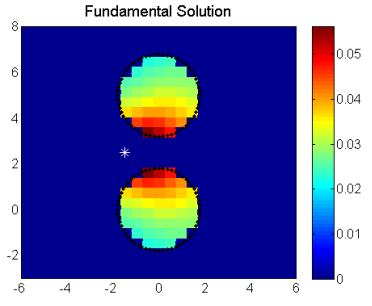

Fundamental Solution

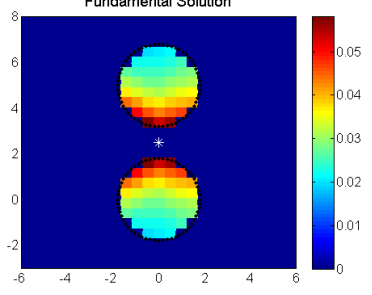

Fundamental Solution

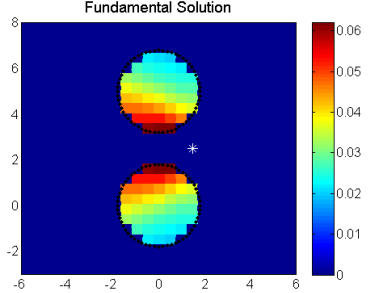

Fundamental Solution

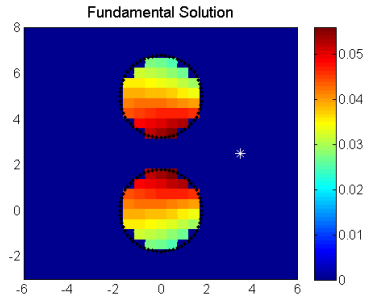

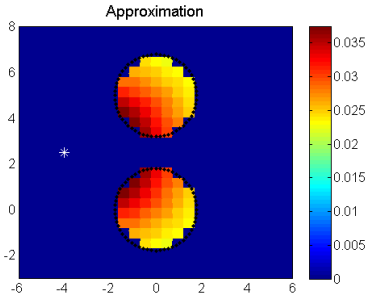

Approximation

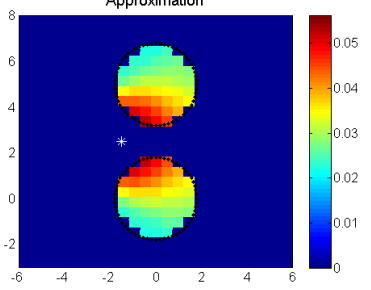

Approximation

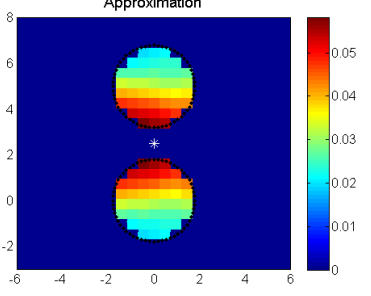

Approximation

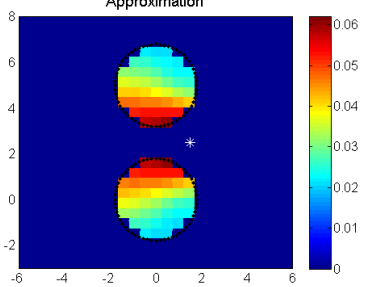

Approximation

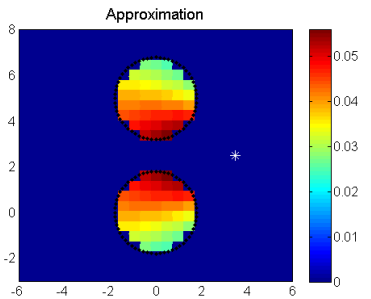

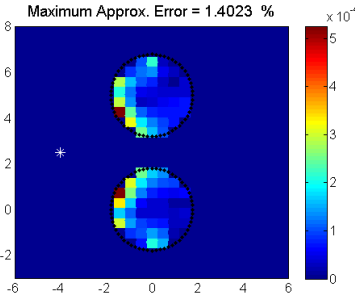

Maximum Approx. Error $=13.0947 \%$

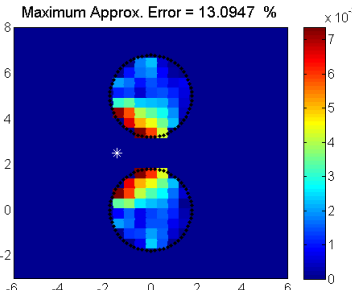

Maximum Approx. Error $=19.5382 \%$

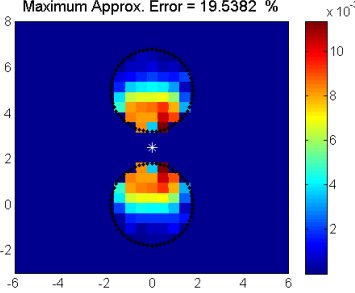

Maximum Approx. Error $=11.2584 \%$

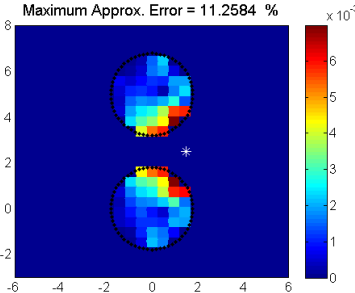

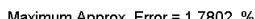

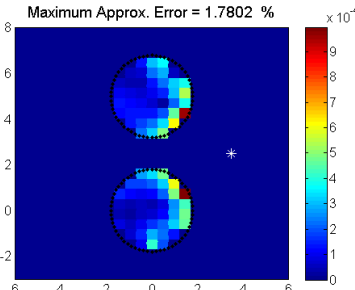

Figure 6.11: In the above figure we take two circles as an approximation domain. In the first and second column we showed the fundamental solution and its approximation between the two parts of the approximation domain $G$ for different grid points, respectively. While in the last column we present the approximation error. It is observed that by moving our grid point between these two circles then our error level increases. In the first two columns the color bar is set to be the maximum value of the fundamental solution while in the third column it depends on the maximum value of the absolute error. 
$G_{1}$ and $G_{2}$. We did our computation on $[-6,6] \times[-6,8]$ with 625 sampling points in the grid. Since we know the size of the unknown obstacle, so as a first step we take the split parts of the approximation domain exactly equal to the known obstacles $D_{1}$ and $D_{2}$, respectively. Here we choose viscosity parameter $\mu=3$. The regularization parameters $\alpha=10^{-12}$ which is quite acceptable. Since we know the position of obstacle so our problem is reduced to a much simpler problem that is the reconstruction of the velocity field only. In this case we fixed our approximation domain so we do not need to use the translation or rotation arguments.

The first line in the Figure 6.10 shows us that we have very good reconstruction of flow field outside the obstacles. Now we enlarge the size of the approximation domain as compared to the known obstacles and again implement the point source method for the whole grid, we have very interesting results. The absolute reconstruction error level increases as one can see in the second line of the Figure 6.10. Third line exhibits the same analysis, i.e., by increasing the size of the approximation domain our error level increases. But interestingly a high error exists only between the divided parts of the approximation domain $G$.

Now we adopt the other way to choose the approximation domain $G$. We take an elliptic approximation domain. In the first step we choose the size of approximation domain large enough so that the two obstacle should lie inside it. On the same grid and same values of the all the parameters which was used before for the divided approximation domain we have almost the same result for the elliptic approximation domain. Fourth and fifth row of Fig. 6.10 shows us that the absolute reconstruction error is high in the center of the two obstacles $D_{1}$ and $D_{2}$.
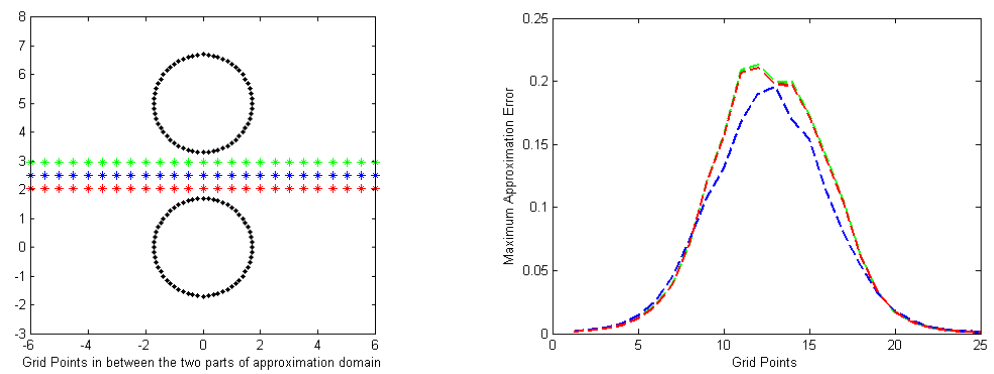

Figure 6.12: To observe the error analysis in the point source method with not a simply connected approximation domain $G$, we took different position of the grid points (left). Thus we have high error level when the source point $x$ lies in between the parts of the approximation domain (right).

The question arises why we have high error in the middle of the two obstacles. Is there any problem with the approximation of the fundamental solution to its single layer potential operator $S g_{x}$ in that area. To analyze this phenomena we again want to have a look of the approximation of the fundamental solution $E(x-\cdot)$ with its 
single layer potential operator $S g_{x}$ given in equation (4.3.19) in the approximation domain $G$. Since we are working with two known obstacles, it is better to split the approximation domain in two parts. For the consistency check we used almost the same procedure which was used before for the one domain analysis. Figure 6.11 tells us very interesting story when our source point is away from the danger area (the area in the middle of obstacles) then the maximum approximation error is much smaller and acceptable. In Figure 6.11 we showed systematically that how the maximum approximation error behaves by putting the source point on different places. It is observed that when the source point $x$ is away from the approximation domain $G$ then the error level is reasonable, while when the source point is between the divided parts of the approximation domain then the error level increased up to $19.5382 \%$. This means that we do not have good approximation in between the split parts of domain $G$. In Figure 6.12 we check out this fact by taking three consecutive lines, in between the two parts of the approximation domain, while each line consists of 25 points. We observed that the error level is high when the source point lies between the divided parts of approximation domain $G$.

Finally, we show how shape reconstructions can be obtained from the flow reconstructions in the second step. The unknown boundary $\partial D$ is the set of points where the total flow is small. Thus, we obtain shape reconstructions by testing

$$
\left|u_{\text {rec }}(x)\right| \leq \kappa
$$

with some appropriately chosen constant $\kappa$ depending on the discretization level. Results are demonstrated in our last Figure 6.13.
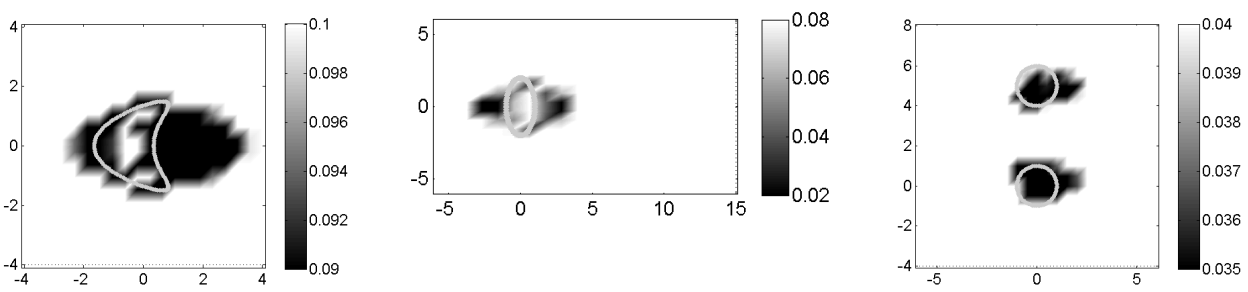

Figure 6.13: We show shape reconstructions for the examples above using the threshold (6.2.10) applied to the flow reconstructions $u_{\text {rec }}$ obtained by the PSM and domain sampling. We observe that we also detect areas of low flow to be potential objects or part of the object, reflecting the practical non-uniqueness of shape reconstructions based on the flow fields.

\subsection{Convergence Test}

We start this section by applying the convergence test to reconstruct the shape of an unknown obstacle in the inverse fluid flow problems. The convergence test is 
basically a variant of the no-response test. The idea of the convergence test is to locate those regions in the grid $\mathcal{G}$ where the velocity field is convergent under the Cauchy criterion as described in section 5.3. We calculate two reconstructed velocity fields $u_{r e c, \alpha_{1}}$ and $u_{r e c . \alpha_{2}}$ for two different regularization parameter $\alpha_{1}$ and $\alpha_{2}$. For this we used the back projection formula (4.3.19) of point source method. In the next step we calculate the mask as defined in equation (6.2.3) for some cut off parameter $\kappa$. With the help of this mask we define an indicator function

$$
\mathfrak{T}_{\kappa}(x):= \begin{cases}1, & \left|u_{r e c, \alpha_{1}}(x)-u_{r e c, \alpha_{2}}(x)\right| \leq \kappa \\ 0, & \text { otherwise }\end{cases}
$$
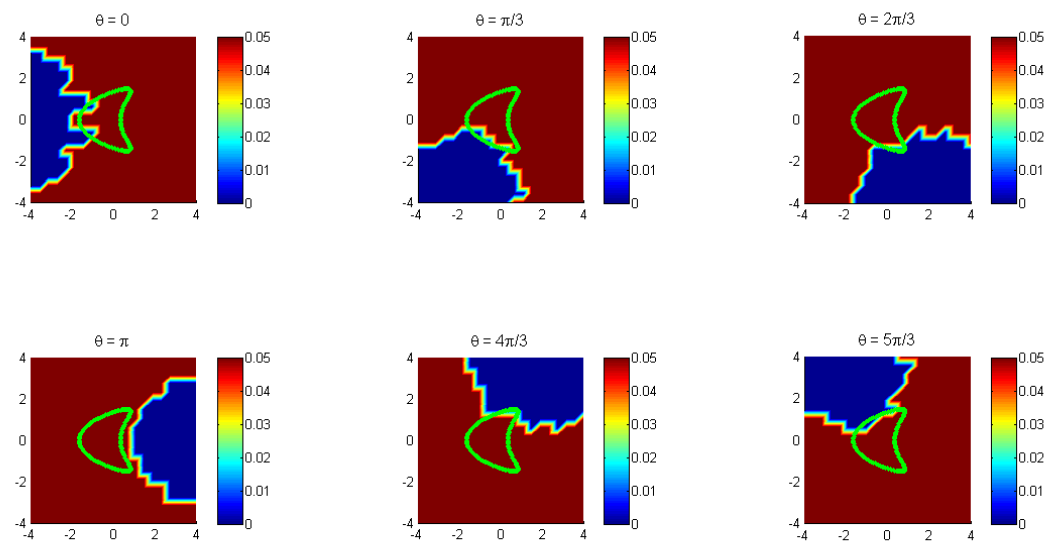

Figure 6.14: This figure shows the six different setting of approximation domain $G$ around a source point $x$. The blue color point out the convergence of the velocity field for source point $x$. Here we take the threshold parameter $\kappa=0.006$.

This indicator function $\mathfrak{T}_{\kappa}$ is used to detect the areas where we do not have the convergence for a particular setting of the approximation domain $G$. We shall denote this non-convergence region as $\mathfrak{D}$. Similarly we calculate the non-convergence region for a different setting of the approximation domain by changing the angle in the rotation matrix. Thus we have $L$ different non-convergent regions for $L$ different directions of the approximation domain for the source point $x$. Obviously, our unknown obstacle is in the non-admissible region of the grid $\mathcal{G}$. Therefore, we take the intersection of all non-convergent regions to obtain the subset of our unknown obstacle $D$, i.e.,

$$
D_{r e c}=\left(\bigcap_{j=1}^{L} \mathfrak{D}_{j}\right)^{c}
$$


We summarize the convergence test in the following algorithm.

Algorithm 6.3. For the inverse fluid flow problems, we reconstruct the shape of unknown obstacle with the help of convergence test using the reconstructed velocity field $u_{\text {rec }}$ of the true velocity $u$ obtained by point source method field on a grid $\mathcal{G}$ by the following steps:

1: Calculate the reconstructed velocity field $u_{r e c, \alpha_{1}}$ and $u_{r e c, \alpha_{2}}$ for two different Tikhonov regularization parameters using point source method as described in first five steps of Algorithm 6.2.

2: Choose a threshold parameter $\kappa$ and calculate the indicator function, defined in equation (6.3.1), for $L$ different desired directions to identify the regions which are convergent under the mask (6.2.3).

3: Finally, the complement of the union of these convergent regions is the subset of the shape of unknown obstacles.

Examples: In the first example we choose a kite shaped obstacle whose parametric representation is given in equation (6.1.2). Following Algorithm 6.3, we first calculates the reconstructed velocity field $u_{r e c}$ for two different regularization parameters $\alpha_{1}=10^{-10}$ and $\alpha_{2}=10^{-11}$. For this we choose a grid $\mathcal{G}$ of size $[-4,4] \times[-4,4]$ with 400 grid points. We did our reconstruction procedure with a circular approximation domain with radius $r=4$ with 150 sampling points. We rotate the approximation domain in six different directions around the source point $x$ (for detail we refer to Section 6.2). Here for the convergence test we choose the viscosity parameter $\mu=5$. According to second step of the Algorithm 6.3, we calculate the indicator function $\mu_{\kappa}$, here we take the threshold parameter $\kappa=0.006$.

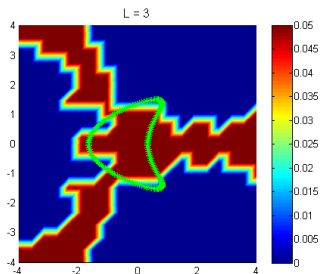

(a)

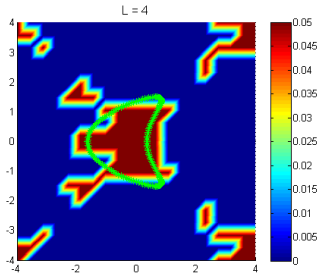

(b)

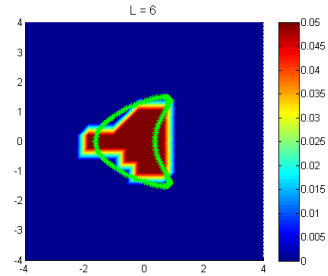

(c)

Figure 6.15: Here we presents the comparison of the reconstruction of obstacle with different choices of rotation parameter $L$. In the last figure we take intersection of the non-convergent areas which are shown in figure 6.14. With the help of first two figures we conclude that for a good reconstruction we need at least six direction of the approximation domain $G$ around the source point $x$.

In Figure 6.14 the red parts exhibits the regions $\mathfrak{D}$ where we have no valid reconstruction, while the area shown by blue points represents the convergent region. 
Once we obtained the non-convergent regions for each different directions then we take the intersection of all these regions and obtained the unknown obstacle $D$, which is shown in the Figure 6.15(c). We did the same experiment by taking the rotation parameter $L=3,4$ and results are shown in Figure 6.15(a) and in Figure 6.15 (b) respectively. Thus with the less number of rotation parameter $L$ we are not able to get the good approximation of unknown obstacle $D$. However with this kind of sampling data, six directions are enough.
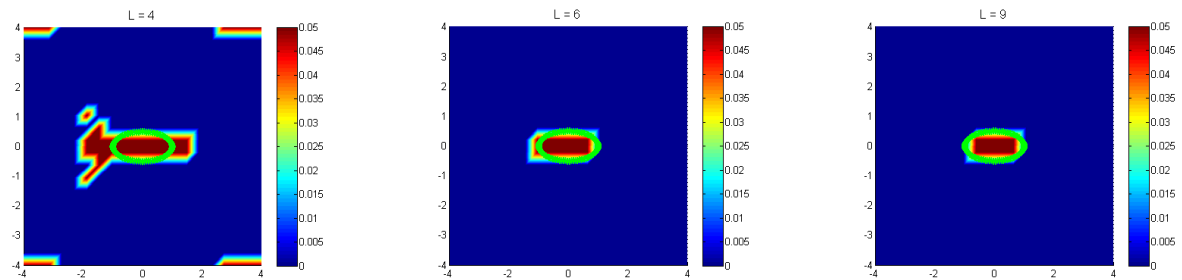

Figure 6.16: We show the shape reconstruction of an elliptic obstacle using the convergence test with the threshold parameter $\kappa=0.0085$. We exhibit the role of rotation parameter $L$. We can see that for a good approximation we need to rotate the approximation domain for at least six directions. For more rotation we approaches to a fine approximation of the obstacle.

To emphasize the importance of rotation parameter $L$ in the convergence test we simulate another experiment to reconstruct an elliptic shape unknown obstacle. With the same sampling data which was taking by the previous example but with the different threshold parameter $\kappa=0.0085$. This is because of an elliptic shaped obstacle. In Figure 6.16 we presents the reconstruction of unknown obstacle $D$ with three different choices of rotation parameter $L$. we observed that with $L=6$ and $L=9$ we have good approximation however with $L=4$ we have non convergent region with the unknown obstacle. 


\section{Chapter 7}

\section{LASSO Scheme}

The goal of this chapter is to reconstruct the shape of unknown obstacles in the inverse fluid flow problem with the help of LASSO scheme. We use the point source method to reconstruct the velocity field to implement the LASSO algorithm.

\subsection{LASSO Algorithm}

LASSO scheme is a newly developed methodology to reconstruct the shape of the unknown obstacles. As compared to domain sampling method this technique insures that our unknown obstacle must lies inside the approximation domain. The main idea of the LASSO scheme is that our approximation domain stretches inside towards the unknown obstacles. Fig. 7.1 demonstrates that for unknown obstacles how our approximation domain stretches inside. For the reconstruction of the shape of more than one obstacle, our approximation domain splits into several sub-domains for searching those unknown obstacles. For example in Fig. 7.3 we can see that our approximation domain $G$ splits into two sub domains $G_{1}$ and $G_{2}$ for searching the unknown domain. Now we explain this procedure in detail.

In point source method, it is always difficult to choose the approximation domain. However this difficulty is resolved by the LASSO scheme. In LASSO scheme we set an approximation domain $G$ large enough so that our unknown obstacle $D$ must contain in it. Although there is no restriction on the choice of approximation domain but here we prefer to choose a circular shaped domain. We discretized the boundary $\partial G$ of the domain $G$ in the $n \in \mathbb{N}$ number of points, i.e., $\left\{p_{1}, p_{2}, p_{3}, \ldots, p_{n}\right\}$. The parametric representation of $\partial G$ is given by

$$
p(t)=\left(p_{1}(t), p_{2}(t)\right)=(r \cos (t), r \sin (t)), \quad t \in[0,2 \pi]
$$

where $r$ is the radius of $\partial G$.

The point source method works under the assumption that our source point $x$ must lies outside the approximation domain $G$, i.e, $x \notin G$. Since we do not know the 
location of the unknown obstacle so we prefer to take the domain $G$ with a larger radius $r$. As $x \notin G$ and the idea of LASSO algorithm is that the approximation domain $G$ stretches inside towards the unknown obstacles, we introduced the concept of parallel surfaces.
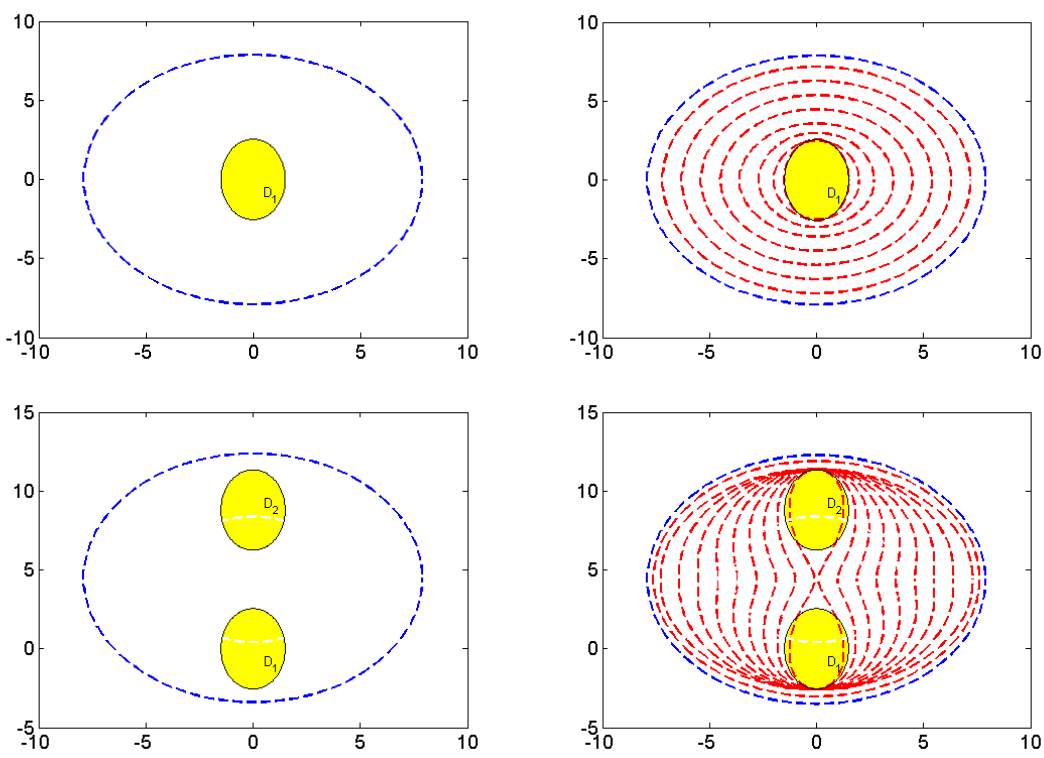

Figure 7.1: In both rows the left hand side represents the unknown Obstacles with the initial guess of approximation domain while the right hand side represents the LASSO scheme which describes that how our approximation domain stretches towards the unknown obstacles

Definition 7.1. Two surfaces are known as the parallel surfaces if the distance $h$ between the corresponding points is constant. Suppose $\Lambda_{1}$ and $\Lambda_{2}$ are two parallel surfaces and let $x \in \Lambda_{1}$ and $z \in \Lambda_{2}$ then we can connect these parallel surfaces by the following equation

$$
\Lambda_{2}:=\left\{z=x+h \nu(x): x \in \Lambda_{1}\right\}
$$

where $\nu$ is the normal vector.

We first construct a new domain $G_{g}$ such that $G \subset G_{g}$. We assume that $x$ belongs to the boundary of domain $G_{g}$, i.e., $\partial G_{g}$. We shall call $\partial G_{g}$ a grid curve and it is dependent on the boundary of the approximation domain $G$ in the following way

$$
\partial G_{g}:=\left\{x=p+h_{g} \nu(p): p \in \partial G\right\}
$$

with some real parameter $h_{g}>0$ which shows the distance between grid curve and the boundary of the approximation domain as shown in the Fig 7.2. Here $\nu(p)$ 
be the normal vector on $\partial G$. After construction of these two curves, as shown in Fig 7.2, we go back to the reconstruction formula (4.3.19) which is obtained by the point source method to reconstruct the velocity field $u_{\text {rec }}$. We implemented the point source method for each source point $x$ which lies on the grid curve $\partial G_{g}$.

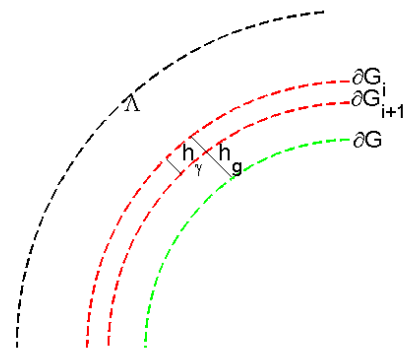

Figure 7.2: Construction of the curves for the LASSO scheme

LASSO scheme is only developed for the reconstruction of the shape of unknown obstacles so we are not interested in the velocity field, but it is obvious that the velocity field must be zero in that area which consists the unknown obstacles. We shall call this area as a significant area where our unknown obstacle $D$ lies. To locate this significant area we define a cut off parameter $h_{a}$ and calculate the absolute velocity

$$
\left\|u_{r e c}\right\|=\sqrt{\left(u_{r e c, 1}\right)^{2}+\left(u_{r e c, 2}\right)^{2}} .
$$

If the absolute value $\left\|u_{r e c}\right\|>h_{a}$ then we stretches the approximation curve $\partial G$ inside towards the unknown obstacles. Now we have a new approximation domain which is defined as

$$
\partial G_{i}=\left\{p^{i}=p^{i-1}-h_{\gamma} \nu\left(p^{i-1}\right) \mathcal{X}: p^{i} \in \partial G_{i}\right\},
$$

with

$$
\mathcal{X}=\left\{\begin{array}{lll}
1 & \text { if } & \left\|u_{r e c}\right\|>h_{a} \\
0 & \text { if } & \left\|u_{r e c}\right\|<h_{a}
\end{array}\right.
$$

here $h_{a}$ is the distance between the boundary of new and old approximation domain, the superscript represents the points of the old and new approximation domain on each step. In equation (7.1.5) the index $i \in \mathbb{N}$ shows the number of steps during the LASSO scheme.

\subsubsection{Smoothness Criteria}

It is clear from the equation (7.1.5) that it is not necessary for the points of approximation domain to reach in significant area simultaneously, since we stretches 


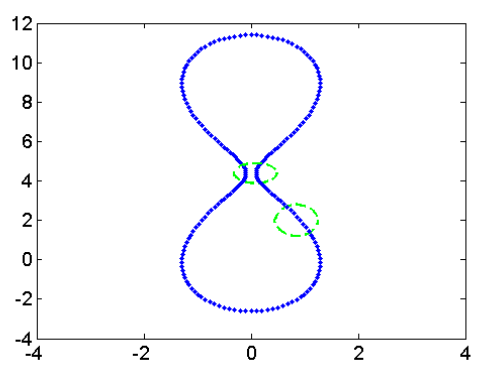

(a)

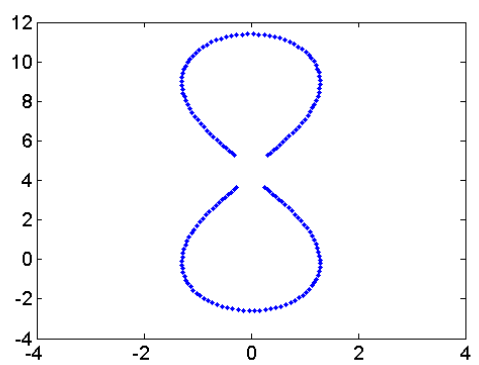

(c)

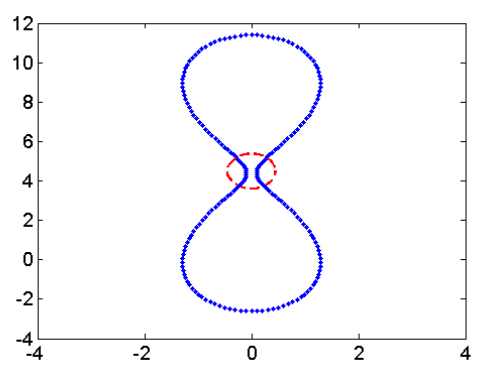

(b)

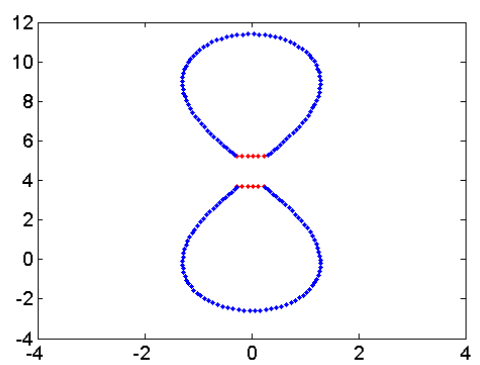

(d)

Figure 7.3: Figure on the left hand side shows that how we locate the points with the help of a circle whose radius is $\sigma$ and right hand side of the figure shows only those points whose mutual distance is more than $\sigma$.

our approximation domain uniformly inside and it is obvious that from one side we may reach in significant area much quicker then the other side. Because of this our approximation domain is no more circular and we have difficulty in each step that our new approximation domain is less smoother than the previous one. Therefore, in some cases, we are not able to construct the curves (7.1.3) and (7.1.5) due to the non-smoothness behavior.

To avoid this difficulty we introduce the concept of smoothness, i.e., for every two neighboring points in the boundary of a curve we choose its middle point and since we have close boundaries so the number of points remains same but we get much smoother curve. We do this process for $n \in \mathbb{N}$ steps until we reached in significant area where the value of absolute velocity $\left\|u_{\text {rec }}\right\|$ is less than the cut off parameter $h_{a}$, then by equation (7.1.5) our new approximation domain is equal to the old one.

As a last step we introduce the following condition,

$$
\left\|p^{i}-p^{i-1}\right\|<h_{s}
$$

to stop the algorithm for a real parameter $h_{s}$, here the superscript represents the points of corresponding domains. 

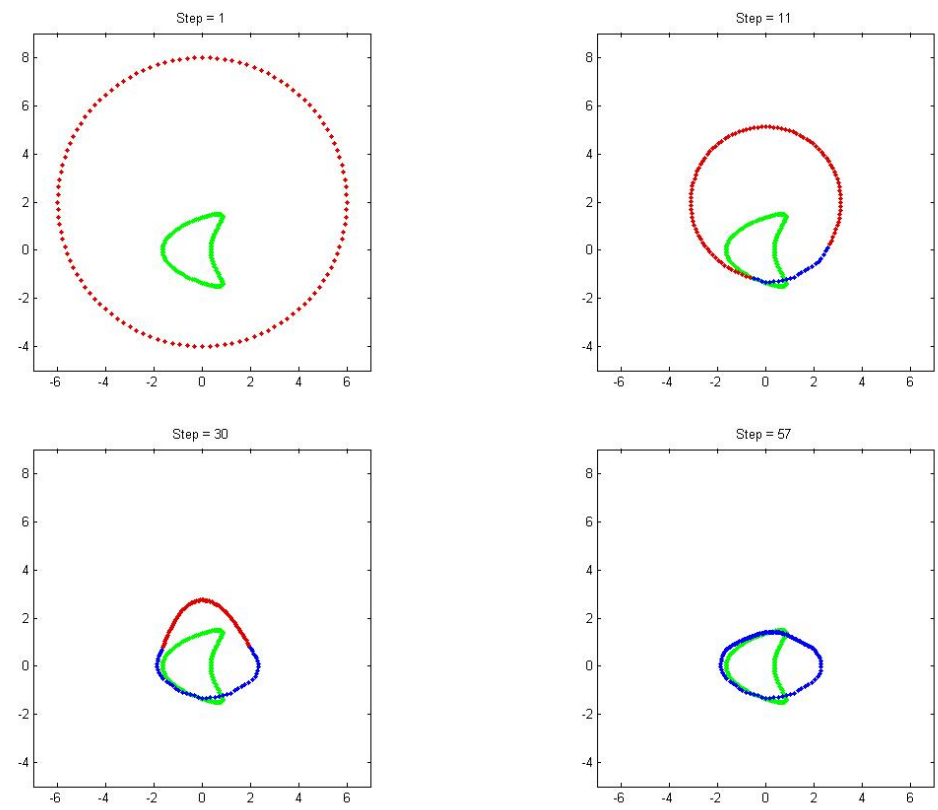

Figure 7.4: Different steps during the reconstruction of a kite shaped obstacle with the help of LASSO scheme. Last figure in the second row gives us the final reconstruction and here we obtained a parabolic wake region behind the obstacle which ensures that our inverse algorithm works properly.

\subsubsection{Domain Splitting}

However, reconstruction of the shape of more than one obstacle, we introduce some modification in LASSO scheme. It is obvious that our approximation domain stretches inside only in that area where $\left\|u_{r e c}\right\|>h_{a}$. Second row of the Fig.7.1 describes that how our LASSO scheme works for two obstacles. The main idea of LASSO scheme for the reconstruction of more than one unknown obstacles is to split our approximation domain $\partial G$ towards those unknown obstacles. More precisely, if we have two unknown obstacles then our approximation domain split into two new approximation sub-domains.

LASSO scheme works under the assumption that our unknown obstacles are not too small. Our approximation domain splits on those points which are close enough to ensures that at least in this area we don't have any unknown obstacle. In Fig. 7.3(b) the red circles show the area where we don't expect any unknown obstacles. In this stage of analysis we split our approximation domain and to split the approximation domain we use the distance check criterion.

For distance check criterion, consider an arbitrary point $p_{j}$ on $\partial G$, we made a subset of $\partial G_{p_{j}}$ which consist all those points of $\partial G$ which lies within the radius of 

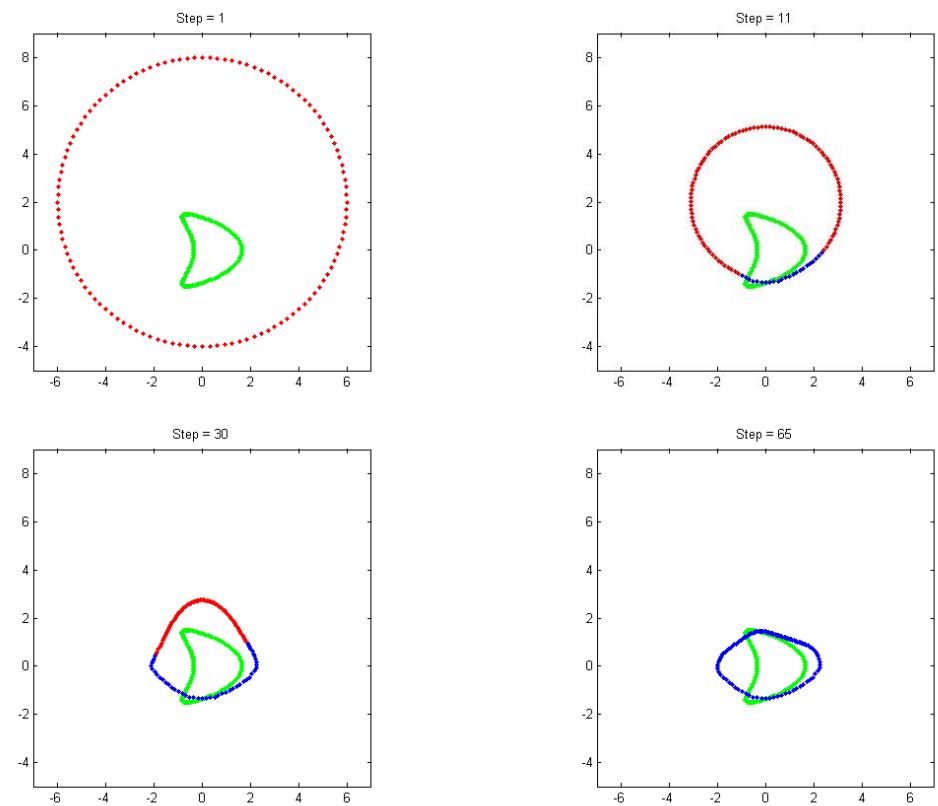

Figure 7.5: Last figure in the second row illustrate that how our parabolic region reduces as compared to Fig. 7.4 behind the obstacle, also in the front side we don't have good reconstruction because of the stagnation points where the velocity goes to zero.

$\sigma$. We may define our subset $\partial G_{p_{j}}$

$$
\partial G_{p_{j}}:=\left\{p \in \partial G:\left|p_{j}-p\right|<\sigma\right\} .
$$

In the next step we leave all those points on $\partial G$ which satisfy the following condition

$$
\left.\left|p_{i}-p_{j}\right|<\sigma \text { for all } i=1,2, \ldots, n \text { and } j=\{1,2, \ldots, n\} \backslash \partial G_{p_{j}}\right\} .
$$

For this we make a circle making its center on $p_{j}$ with radius $\sigma$. We translate this circle for all points on approximation domain and check the above condition (7.1.9). In Fig. 7.3(a) we showed symbolically that how we searched the points which satisfied the distance check condition (7.1.9), and once we have all those points which does not belong to the arbitrary circle then obviously we have two open curves which are the subset of our approximation domain as shown in Fig. $7.3(\mathrm{c})$.

In the next step we connect the respective ends of the open curves by the addition of some points and then applying the smoothing process as explained above. Thus we have two sub domains $G_{1}$ and $G_{2}$ with there boundaries $\partial G_{1}$ and $\partial G_{2}$ consisting of $\left\{p_{1}, p_{2}, \ldots, p_{l}\right\}$ and $\left\{p_{1}, p_{2}, \ldots, p_{q}\right\}$ number of points with $l<m$ and $q<m$ respectively. Now we have two approximation domains instead of one and each domain 

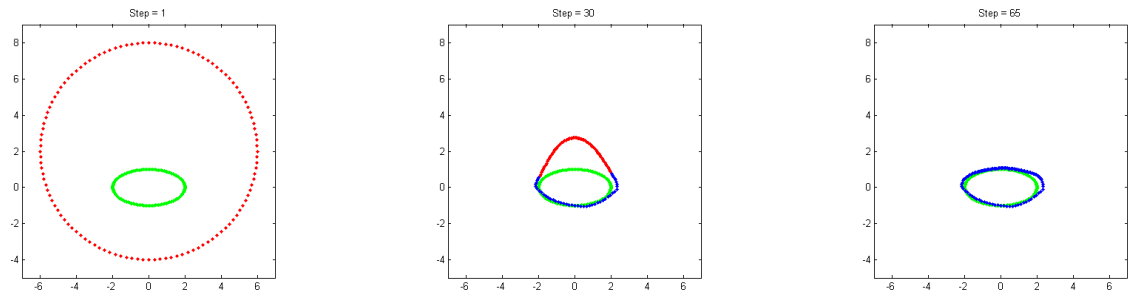

Figure 7.6: In this figure we showed that if we want to reconstruct the obstacle, which is of parabolic form from both sides, then we have very good results

contains one unknown obstacle. Now we apply point source method again on these curves simultaneously and follow the same procedure for $\partial G_{1}$ and $\partial G_{2}$ as we did for the reconstruction of the shape of one unknown obstacle. Fig. 7.8(f) gives us the final reconstruction. We now summarize all the above methodology in the following algorithm.

Algorithm 7.2. We implement the LASSO scheme for the reconstruction of the shape of unknown obstacles in inverse fluid flow problems using point source method by the following steps:

1: Choose a circular reference domain $G$ large enough so that our unknown obstacle lies inside of $G$

2: Making use of equation (7.1.3), construct $\partial G_{g}$ on a distance $h_{g}$ from the approximation domain

3: Calculate absolute velocity $\left\|u_{r e c}\right\|$ at each point of the approximation domain while the source point lies on $\partial G_{g}$

4: If the value $\mathcal{X}=1$ for all or some points on $\partial G$ then using equation (7.1.5) construct a new approximation domain which stretches inside for a step size $h_{\gamma}>0$

5: Apply smoothness criterion to make the new approximation domain smooth enough

6: For the reconstruction of one unknown obstacle, repeat steps 2-5 until we have $\left\|u_{r e c}\right\|<h_{a}$ for all points and by equation (7.1.3). Once this criterion is satisfied the new and old approximation domains coincide and the algorithm stops

7: For the reconstruction of two obstacles, set a distance check criterion which is defined in equation (7.1.9)

8: Neglect all those points which satisfy the distance check criterion and we are left with two open curves

9: Close these open curves by addition of points

10: Now again apply the smoothness criterion on these closed curves

11: Apply point source method on the curves obtained by steps 7-10 Following the stopping criterion given in equation (7.1.7) we reach the final reconstruction 

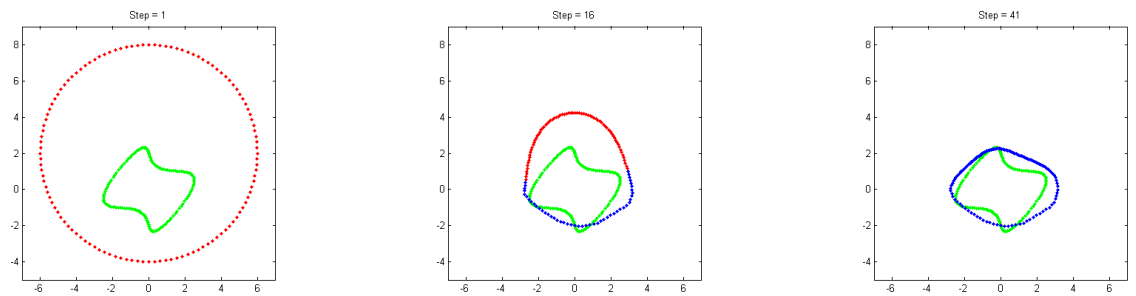

Figure 7.7: In this example we observed the parabolic wake region behind the obstacle

It is discussed before that the viscosity parameter has an effective role in Oseen flow. With the number of experiments it is observed that for the good reconstruction of flow field and the shape of unknown obstacle, the value of viscosity parameter must be larger than or equal to one.

\subsection{Numerical Examples}

As a first example we choose a famous kite shaped obstacle as an unknown domain $D$. In this example we take the value of the viscosity parameter $\mu=12$. The parametric representation of its boundary is given in equation (6.1.2). Following the algorithm 7.2, we choose a circular approximation domain $G$. We discretized the boundary of approximation domain $\partial G$ and the measurement boundary $\Lambda$ into 120 and 200 number of points respectively. In this example and all other examples in which we implement LASSO scheme for the reconstruction of unknown obstacles, we choose the regularization parameters $\alpha=10^{-9}$. We took the value of cut off parameter $h_{a}=0.06$ which is quite reasonable. We take the value of $h_{s}=0.01$ to implement the condition (7.1.7) to stop the algorithm. For making the grid domain $\partial G_{g}$ with the help of (7.1.3) we choose the distance parameter $h_{g}=0.7$ which ensures that our source point must lie outside the approximation domain. In the whole process we took the smoothness parameter is too strong. Fig. 7.4 illustrate the different steps of LASSO scheme for the reconstruction. In figure 7.4 the blue points are those points on which the absolute velocity is less than $h_{a}$. We can see that how our approximation domain stretches towards the unknown obstacle step by step. Thus after 57 steps we reconstruct the shape of the unknown obstacles. Due to the wake region we can not expect any velocity behind the obstacle, and since our algorithm works with the velocity of the fluid so by the definition (7.1.5) and the condition (7.1.7) our algorithm stops. And because of this we do not have the good reconstruction behind the obstacle. However, we have very good reconstruction of the obstacle from the front side which we can see in the second row (right) in Fig. 7.4 .

To observe this analysis in more detail we implement the LASSO scheme to re- 


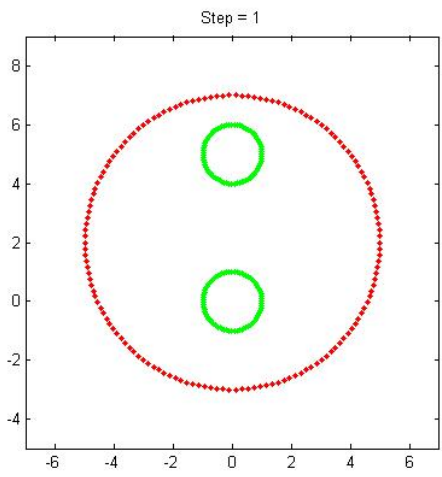

(a)

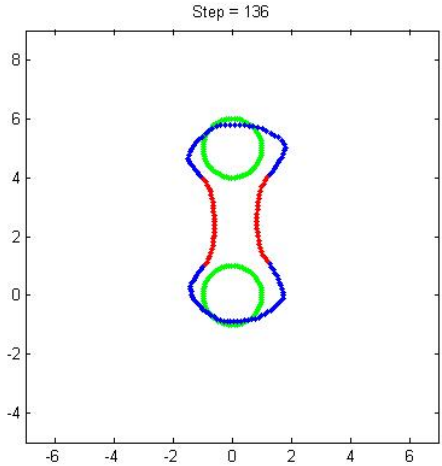

(c)

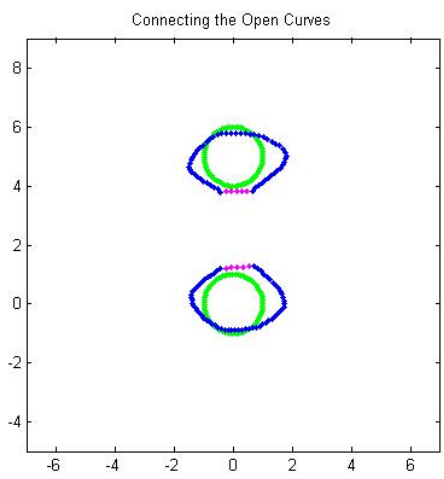

(e)

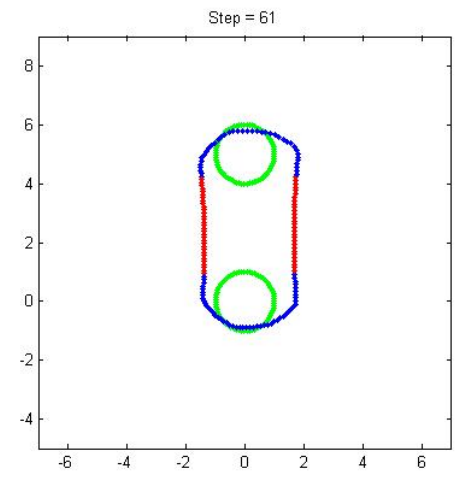

(b)

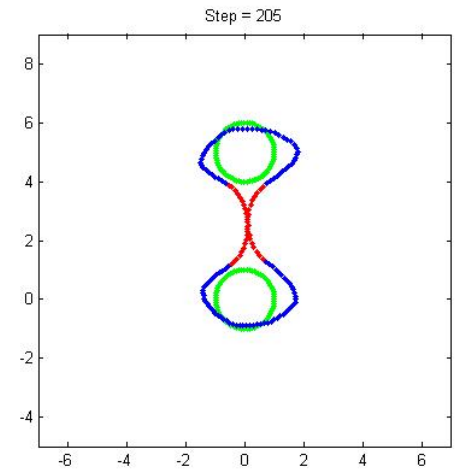

(d)

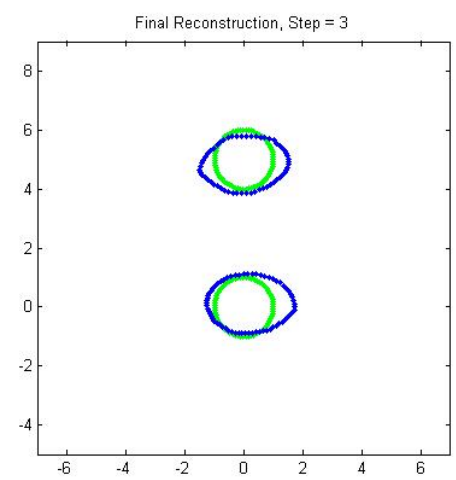

(f)

Figure 7.8: In the first two rows we presents the different steps that how our approximation domain $\partial G$ stretches inside for the reconstruction of two unknown obstacles and in the third row we implement the splitting argument when the points in $\partial G$ satisfy the splitting condition (7.1.7) 
construct the different shaped obstacles. For example if we rotate the kite shaped obstacle to 180 degrees, while the flow is coming from the right side, then we can see that in Fig. 7.5 that due to the parabolic behavior opposite to the flow direction we have very small wake region, but at the same time we do not have good reconstruction in the front side because of the large number of stagnation points, where the velocity goes to zero as well.

For this we take an elliptic obstacle an as unknown (see Fig. 7.6) and we can see that we have very good reconstruction of the obstacle. Since on the front side the number of stagnation points are very less and from the back side we have small wake region, this gives us very good reconstruction of the unknown obstacle. We observed the same results when we choose a very different shaped obstacle (see in Fig. 7.7) whose parametric representation is given by

$$
x(t)=(2 \cos t+0.5 \cos 3 t, 2 \sin t+0.5 \cos 3 t) \quad t \in[0,2 \pi] .
$$

In this obstacle, due to the parabolic behavior on both sides, we have very good reconstruction. In fact in this analysis we observed that for the inverse flow problems wake region and the stagnation points play very important roles for the reconstruction of the shape of unknown obstacles.

Now we demonstrate the beauty of LASSO scheme to reconstruct the two unknown circular obstacles. The idea for reconstructing the two unknown obstacles is almost same as for the one obstacle. As the first step we choose a circular approximation domain $\partial G$ with radius 6 . This time we discretized $\partial G$ in 150 number of points. We discretized the data domain in 200 number of points. In Fig 7.8, the first picture in the first row show us the initial setting for the LASSO scheme, where the green circles are our unknown obstacles. In Fig.7.8, the red points are those points where our absolute velocity is greater then the cut off parameter $h_{a}=0.05$ while the blue points are those which does not fulfill this condition. We took strong smoothness criterion to start the algorithm. Because of the condition (7.1.7) algorithms stops in a very initial stage ( $\mathrm{step}=61$ ) shown in the Fig. 7.8(b). As the red points in the Fig. 7.8(b) are in the straight line and it is not possible to implement the condition (7.1.9) for getting a new approximation domain. To avoid this difficulty we now weaken our smoothness criterion and again implement the LASSO scheme. By using this technique we can move inside towards the unknown obstacle. After reducing the value of smoothing parameter we reached to Fig 7.8(d) in step $=205$.

Now the most important step in the LASSO scheme for the reconstruction of more than on unknown obstacles comes in which we split our approximation domain by using the distance check criterion (7.1.9). We took the value of $\sigma=0.04$, the cutoff parameter for the distance check criterion. With the help of distance check criterion we ignore all those points which lies within the radius $\sigma=0.04$. With this our approximation domain is divided into two open curves. In the next step we connect these two open curves by introducing some points with the help of first and 


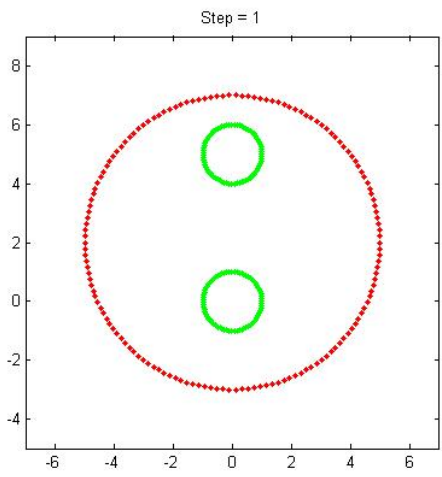

(a)

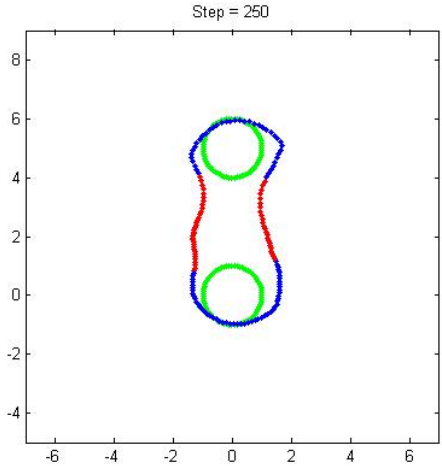

(c)

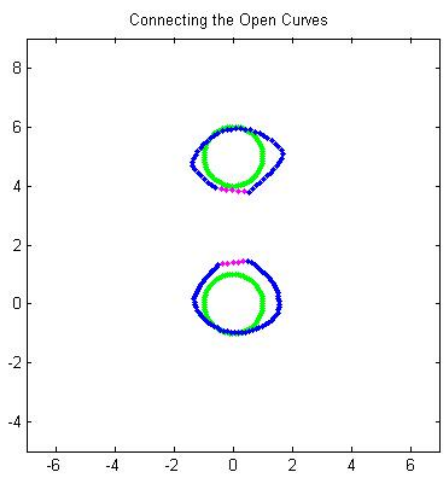

(e)

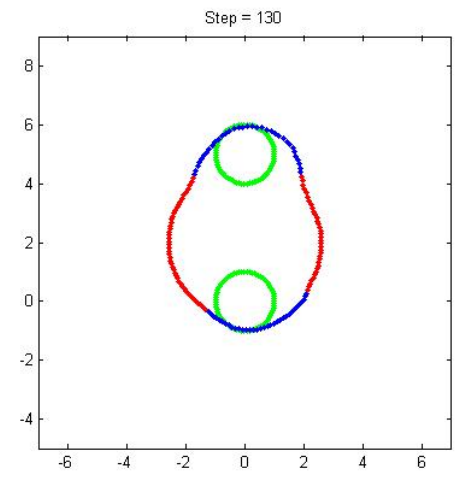

(b)

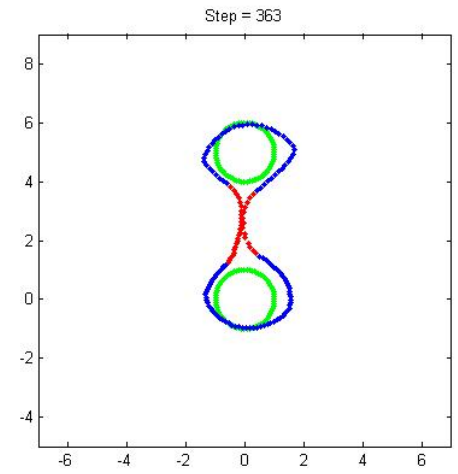

(d)

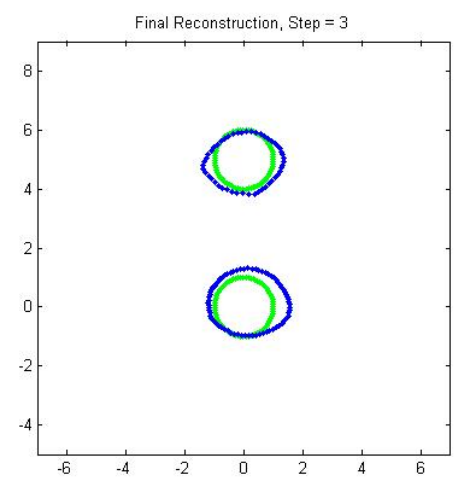

(f)

Figure 7.9: In the first two rows we presents the different steps that how our approximation domain $\partial G$ stretches inside for the reconstruction of two unknown obstacles and in the third row we implement the splitting argument when the points in $\partial G$ satisfy the splitting condition (7.1.7). The difference from the Fig. 7.8 is only that we use the smoothing parameter $\beta=1$ from the start of the program. 


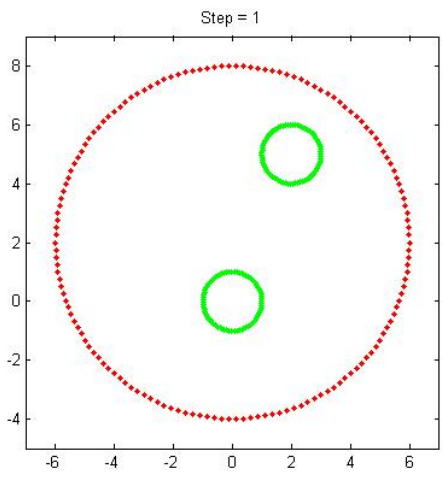

(a)

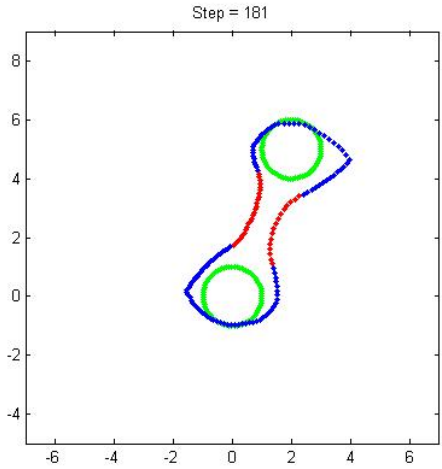

(c)

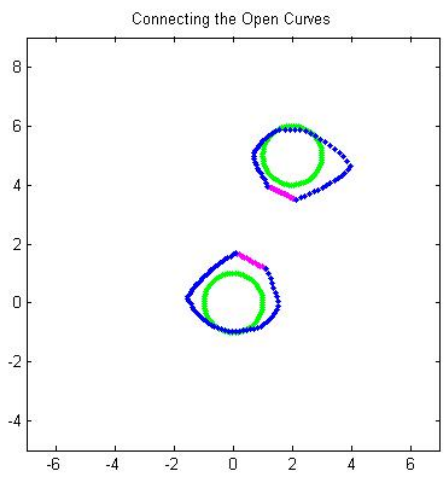

(e)

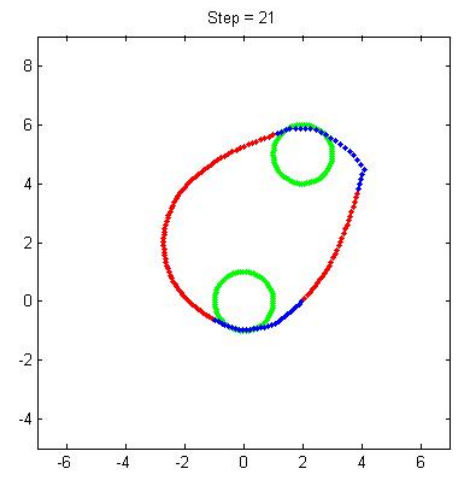

(b)

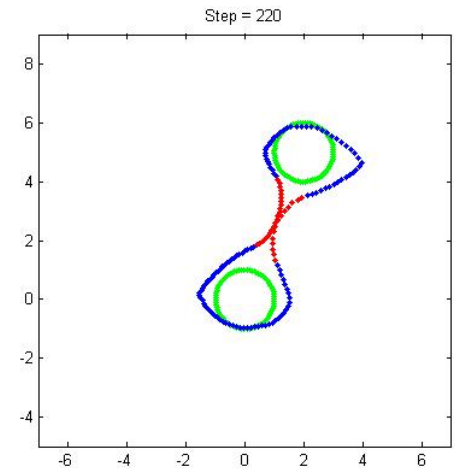

(d)

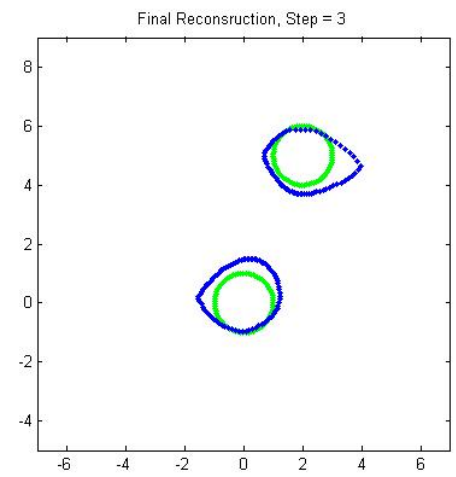

(f)

Figure 7.10: Here we implemented LASSO scheme on a different set up of unknown obstacles. In the first two rows we showed how the scheme works and in the third row we connect the open curves, which we obtained after splitting, and then apply the point source method on each component of the approximation domain separately to obtain the final reconstruction. 
last points of the respective open curves. In Fig. 7.8(e) the blue points represents the open curves while the points in pink color are introduced for connecting these open curves. Now we again implement the PSM Thus in principle we have the location where the unknown obstacle lives as showed in Fig. 7.8(e), but to get the final reconstruction of the shape of the unknown obstacles we need to implement the point source method on these newly developed parts of the approximation domain. We use the same parameters as we used before but this time just after 3 steps we have the final reconstructed shapes of the obstacles. Finally we have the final reconstruction of the unknown obstacles as shown in Fig. 7.8(f).

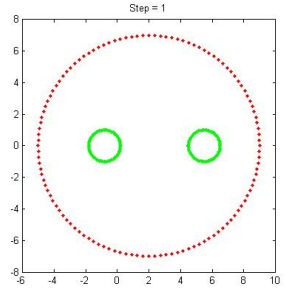

(a)

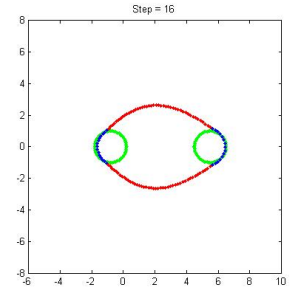

(b)

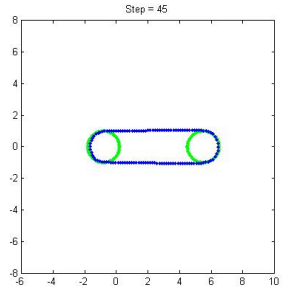

(c)

Figure 7.11: In this figure we investigate the applicability of LASSO algorithm when two unknown obstacles are setting in a same horizontal line and in the direction of flow field. For the same cutoff parameter we we can not go inside even we reduced the smoothing parameter because of the wake region.

Here off course the wake region and the stagnation points play again an important role which can be seen in the 7.8(f). We should mention that there should be another factor, the smoothing parameter, which has an important role here and will be discuss in details. To observe the role of this factor we implement the LASSO scheme on the same setup but the only difference is we take the value of smoothing parameter $\beta=1$, the smallest value, then we reached in 363 steps rather than 205 steps, until we applied the distance check criterion. Thus with this value of smoothing parameter $\beta$ we have to do 158 number of steps more, however if we compare the reconstruction quality then it shows not a big difference (see Fig. 7.8(f))and even we lose the wake region as well. So we can conclude that we must take a strong smoothing criterion in the start.

Now we change the position of the unknown obstacles and putting them in a row in the direction of the flow as shown in Fig. 7.11(a). In this example we start the algorithm with strong smoothing parameter $\beta=40$. After 45 steps the algorithm stops and we are in the position shown in the figure 7.11(c). As compared to the previous examples we have different results. We have only blue points which is not a good sign this means that we cannot go inside even if we reduced the value of the smoothing parameter. As our cutoff parameter $h_{a}$ depends on the value of 
velocity of the fluid. And from physical point of view we do not expect a good flow between these two obstacles and so there is no velocity and hence $h_{a}$ should be zeros and with this our new approximation domain is equal to the old one and algorithm stops. Thus in this case we can not have a good reconstruction. In Fig. 7.10 we again changed the position of the obstacles and implement the LASSO scheme and we have very good results.

Thus in principle we can say that the LASSO scheme can be applied for the reconstruction of the shaped of one or two unknown obstacles. We can generalized this idea for the reconstruction of more than two unknown obstacles. 


\section{Part III}

\section{Appendix}





\section{Appendix A}

\section{Background from Fluid Dynamics}

Fluid mechanics is one of the oldest and richest branch of mechanics and applied mathematics. It deals with the behavior of fluids both at rest and in the motion. Fluid mechanics has been studied, formally or informally, since the beginning of the recorded history. We distinguish fluids from the solids by adopting the criterion that fluids deform continuously when a shear stress is applied while in the solids we could not see the continuous deformation. This criterion is independent from the size of shear stress. We focus our study to one of the most important branch of fluid mechanics, i.e., fluid dynamics in which we deal with the fluid flow and its applications.

It is well known that the forces exerted on the fluid element may be classified into body forces and surface forces. The forces which are distributed over the entire volume of the fluid are known as body forces for example the gravitational and electromagnetic forces. Surface forces are forces which act on the boundaries of the medium through direct contact.

Assume that a fluid is moving with velocity $u$ within the region $\Omega$ of the two dimensional space $\mathbb{R}^{2}$. We consider a portion $\delta \vec{A}$ of the surface $A$ of the flowing fluid passing through the point $P$. Let $\hat{n}$ be the unit normal vector on the surface $\delta \vec{A}$. The force $\delta \vec{F}$ exerted on the surface $\delta \vec{A}$ can be resolved into two components, normal $\delta \vec{F}_{n}$ and tangential $\delta \vec{F}_{t}$. Now we can define the stress which is basically a measure of the average force per unit area of a surface. We can resolve stress into two components, the normal stress $\sigma_{n}$ and the shear stress $\tau_{n}$. These stress are defined as

$$
\begin{aligned}
\sigma_{n} & =\lim _{\delta \vec{A} \rightarrow 0} \frac{\delta \vec{F}_{n}}{\delta \vec{A}_{n}} \\
\tau_{n} & =\lim _{\delta \vec{A} \rightarrow 0} \frac{\delta \vec{F}_{t}}{\delta \vec{A}_{n}}
\end{aligned}
$$


Here the subscript $n$ tells us that the stresses are associated with the surface, $\delta \vec{A}$, through the point $P$, on which we have the normal vector $\nu$. This means that for any other surface through $P$, the value of stresses may differ.

While studying fluid dynamics, viscosity parameter plays a significant role. Informally, viscosity is the quantity that describes the fluid's resistance to flow, or we can say that it measures the thickness of the fluid. For example, water has low viscosity as compared to honey as water is much thicker than honey. In other words we can say that the viscosity is the internal friction of the fluid. Formally, viscosity is the ratio of the shearing stress to the velocity gradient in a fluid, which is being deformed by either shear stress or normal stress.

\section{A.1 Equation of Continuity}

Let the fluid be moving with the velocity $u(x, t)$ at time $t$ in a fixed sub-domain $\Omega_{0}$ of $\Omega$. Let $\nu$ denotes the unit normal vector on the boundary $\partial \Omega_{0}$ of $\Omega_{0}$. The mass of the $\Omega_{0}$ is given by $\int_{\Omega_{0}} \rho d x$, where $\rho(x, t)$ is the mass density of the fluid dependent of $x$ and $t$ and $d x$ denotes the elementary volume. The decrease of mass per time unit is given by

$$
-\frac{d}{d t} \int_{\Omega_{0}} \rho d x
$$

while the total mass leaving $\Omega_{0}$ through its boundary $\partial \Omega_{0}$ per time unit is given by

$$
\int_{\partial \Omega_{0}} \rho u \cdot \nu d x
$$

Thus we have the following mass balance equation

$$
-\frac{d}{d t} \int_{\Omega_{0}} \rho d x=\int_{\partial \Omega_{0}} \rho u \cdot \nu d s
$$

Making use of the divergence theorem on the right hand side we obtain

$$
-\frac{d}{d t} \int_{\Omega_{0}} \rho d x=\int_{\Omega_{0}} \nabla \cdot(\rho u) d x
$$

or

$$
\int_{\Omega_{0}}\left(\frac{\partial \rho}{\partial t}+\nabla \cdot(\rho u)\right) d x=0 .
$$

Since $\Omega_{0}$ is an arbitrary sub-domain of the region $\Omega$, we can rewrite equation (A.1.1) as

$$
\frac{\partial \rho}{\partial t}+\nabla \cdot(\rho u)=0 .
$$


Equation (A.1.2) is known as the continuity equation. In this document we are interested in incompressible fluids in which the volume of any fluid element is time invariant when moving with flow i.e.,

$$
\frac{d}{d t} \int_{\Omega_{0}} d x=0
$$

On the other hand with the help of Lagrangian technique, which will be discussed in detail while deriving the equation of motion, we have the following equation for any kind of fluid, whether it is compressible or incompressible,

$$
\frac{d}{d t} \int_{\Omega_{0}} d x=\int_{\Omega_{0}} \nabla \cdot u d x
$$

For an arbitrary $\Omega_{0}$, equations (A.1.3) and (A.1.4) implies the following incompressibility condition

$$
\nabla \cdot u=0
$$

If we assume that the mass density $\rho$ is a constant function of $x$ and $t$ over the whole flow region then the continuity equation (A.1.2) reduces to the incompressibility condition (A.1.5). However the reciprocal is not true. For interested readers we refer to [3].

\section{A.2 Equation of Motion}

Considering a fluid element occupying domain $\Omega$ at time $t$. The fluid element moves with the flow velocity $u(x, t)$ and reaches the domain $\Omega^{\prime}$ at time $t^{\prime}$, while obviously $t^{\prime}>t$. Newton's second law of motion applied on the fluid element gives us

$$
\frac{d}{d t} \int_{\Omega} \rho d x=\int_{\Omega} \rho f d x+\int_{\partial \Omega} \mathcal{S} d s
$$

Where $f$ denotes a density of volume forces per mass unit and $\mathcal{S}$ denotes a density of surface forces per surface unit. The surface forces $\mathcal{S}$ are expressed with a $2 \times 2$ symmetric tensor $\sigma$, known as stress tensor in the following way

$$
\mathcal{S}=\sigma \nu
$$

Here $\nu$ is an outward unit normal vector and the stress tensor $\sigma$ is the combination of normal stresses $\sigma_{n}$ and shear stresses $\tau_{n}$ corresponding to the normal and tangential forces applied on the some surface element, respectively. In short we can say that the surface forces are the result of the forces of $\sigma$ applied in the outward unit normal vector $n$. Following [5] and [15] we can write the stress tensor for the viscous fluid as

$$
\sigma=-\tilde{p} I+A
$$


Here $\tilde{p} I$ is the in determinant part of the stress tensor, where $\tilde{p}$ is the pressure and $I$ is the identity matrix. For Newtonian fluids the matrix $A$ is given as

$$
A=2 \tilde{\mu}\left(D-\frac{1}{3} \nabla \cdot u I\right)+\tilde{\mu}_{1} \nabla \cdot u I .
$$

As a remark we would like to mention that the viscosity parameters $\tilde{\mu}$ (dynamic viscosity) and $\tilde{\mu}_{1}$ (second viscosity) play an important role in the modeling of equation (A.2.4). The first term of the right hand side represents the viscous effects associated to volume invariant deformation while the second term is responsible for the volume dilations due to viscous effects. In general viscosity coefficients are the functions of temperature, but we are interested only in isothermal flows in which the temperature is uniformly constant in the flow region $\Omega$. With the help of equation of continuity (A.1.5), the equation (A.2.4) is reduced to

$$
A=2 \tilde{\mu} D .
$$

Here $D$ is the deformation rate tensor defined as

$$
2 D=\nabla u+(\nabla u)^{T}
$$

Now making use of equation (A.2.2) and with the help of the divergence theorem, the second term on the right hand side of equation (A.2.1) can be written as

$$
\int_{\partial \Omega} \mathcal{S} d s=\int_{\Omega} \nabla \cdot \sigma d x
$$

From equations (A.2.3) and (A.2.4), keeping in mind that $\nabla \cdot a I=\nabla a$, we obtain

$$
\nabla \cdot \sigma=-\nabla \tilde{p}+\tilde{\mu} \Delta u
$$

Here $\Delta$ is a Laplace operator. Insert (A.2.7) in equation (A.2.6) we finally obtain

$$
\int_{\partial \Omega} \mathcal{S} d s=\int_{\Omega}(-\nabla \tilde{p}+\tilde{\mu} \Delta u) d x .
$$

Now we evaluate the left hand side of equation (A.2.1). We denote the time difference by $\Delta t=t^{\prime}-t$, then by the classical definitions of derivatives, we have

$$
\frac{d}{d t} \int_{\Omega}(\rho u)(x, t) d x=\rho \lim _{\Delta t \rightarrow 0} \frac{1}{\Delta t}\left[\int_{\Omega^{\prime}} u\left(x^{\prime}, t^{\prime}\right) d x^{\prime}-\int_{\Omega} u(x, t) d x\right] .
$$

For a small $\Delta t$, we may have the infinitesimal one-parameter coordinate transformation in the following way

$$
x^{\prime}=x+\Delta t u(x, t)+O\left(\Delta t^{2}\right) .
$$


Neglecting terms of order $O\left(\Delta t^{2}\right)$, we have

$$
x^{\prime}=x+\Delta t u(x, t) .
$$

Using equation (A.2.10), we change the variables of the first integrand on the right hand side of equation (A.2.9), such as

$$
\begin{aligned}
\frac{d}{d t} \int_{\Omega}(\rho u)(x, t) d x=\rho \lim _{\Delta t \rightarrow 0} \frac{1}{\Delta t}\{ & \int_{\Omega} u(x+\Delta t u(x, t), t+\Delta t) \operatorname{det}\left(\frac{\partial x^{\prime}}{\partial x}\right) d x \\
& \left.-\int_{\Omega} u(x, t) d x\right\}
\end{aligned}
$$

In the above equation $\frac{\partial x^{\prime}}{\partial x}$ denotes the Jacobian matrix of the transformation defined in (A.2.10). With straight forward calculation starting from equation (A.2.10) yields

$$
\frac{\partial x^{\prime}}{\partial x}=I+\Delta t \nabla u
$$

Now taking the determinant of equation (A.2.12), we obtain

$$
\left|\frac{\partial x^{\prime}}{\partial x}\right|=1+\Delta t \nabla \cdot u+\Delta t^{2} \sum_{i<j}\left(\frac{\partial u_{i}}{\partial x_{i}} \frac{\partial u_{j}}{\partial x_{j}}-\frac{\partial u_{i}}{\partial x_{j}} \frac{\partial u_{j}}{\partial x_{i}}\right), \quad i, j=1,2(\mathrm{~A} .2 .13)
$$

Here we use the notation $\nabla \cdot u=\sum \frac{\partial u_{i}}{\partial x_{i}}$. Under the assumption that $\Delta t$ is small, we again neglect the terms with $\left(\Delta t^{2}\right)$ in equation (A.2.13) and with incompressibility we have

$$
\frac{\partial x^{\prime}}{\partial x}=1
$$

Now using the first order Taylor series expansion on $(\rho u)(x+\Delta t u, t+\Delta t)$ gives

$$
u(x+\Delta t u, t+\Delta t)=u+\Delta t(u \cdot \nabla) u+\Delta t \frac{\partial u}{\partial t} .
$$

Thus in the view of equations (A.2.14) and (A.2.15), equation (A.2.11) takes the following form

$$
\frac{d}{d t} \int_{\Omega} \rho u d x=\rho \lim _{\Delta t \rightarrow 0} \frac{1}{\Delta t}\left\{\int_{\Omega}\left(u+\Delta t(u \cdot \nabla) u+\Delta t \frac{\partial u}{\partial t}\right) d x-\int_{\Omega} u d x\right\}(
$$

Applying the limit on equation (A.2.16) we are left with

$$
\frac{d}{d t} \int_{\Omega} \rho u d x=\rho \int_{\Omega}\left((u \cdot \nabla) u+\frac{\partial u}{\partial t}+u(\nabla \cdot u)\right) d x
$$


Making use of equation of continuity (A.1.5), the last equation can also be written as

$$
\frac{d}{d t} \int_{\Omega} \rho u d x=\rho \int_{\Omega} \frac{\partial u}{\partial t}+(u \cdot \nabla) u d x
$$

Now, finally substituting back (A.2.8) and (A.2.17) in equation (A.2.1) and taking advantage of an arbitrary domain $\Omega$ we have the following equation of motion for incompressible fluids

$$
\rho \frac{\partial u}{\partial t}+\rho(u \cdot \nabla) u=-\nabla \tilde{p}+\tilde{\mu} \Delta u+\rho f .
$$

We modify the above equation (A.2.18) such that,

$$
\frac{\partial u}{\partial t}+(u \cdot \nabla) u=-\nabla p+\mu \Delta u+f
$$

here $p=\tilde{p} / \rho$ and $\mu:=\tilde{\mu} / \rho$ is the kinematic viscosity.

Definition A.1. The Navier-Stokes equation for isothermal incompressible Newtonian fluids are defined by the system of equations consisting of

- The equation of continuity (A.1.5) and the

- The equation of motion (A.2.19)

In order to analyze the behavior of the fluids in detail we need some conditions that strongly depend on the type of the region $\Omega$ of the flow. In this monograph we are considering two types of regions. First type of region is bounded while the other type of the region is the compliment of a bounded region. For the first case we only need boundary condition at the bounding walls $\partial \Omega$ of the region $\Omega$, i.e.,

$$
u(x)=u_{*}(y), \quad y \in \partial \Omega .
$$

While for the second case, in addition to condition (A.2.20), we impose the condition that our velocity field $u(x)$ at large spatial distances tends to some vector $u_{\infty}$ such that

$$
\lim _{|x| \rightarrow 0} u(x)=u_{\infty}(y)
$$

The second term on the left hand side of equation (A.2.19) is responsible for the non-linearity of the Navier-Stokes equation. In the last two centuries it is a challenging task for mathematicians to get explicit solutions of these equations. However, there are several numerical methods developed in computational fluid dynamics (CFD) to deal with the Navier-Stokes equation [8]. 
In this monograph we shall investigate the inverse problems of viscous incompressible steady fluids with slow motion. The hypotheses of slow motion means that the viscous forces are much stronger than the inertial forces, i.e.,

$$
\frac{(u \cdot \nabla) u}{\mu \Delta u} \rightarrow 0
$$

Thus we can neglect the non-linear term from equation (A.2.19) to make it linear. For mathematical justification we linearize the Navier-Stokes equation by standard tools. Assume that $\left(u_{0}, p_{0}\right)$ is the generic solution of the Navier-Stokes equations. This means that $\left(u_{0}+\lambda u(x), p_{0}+\lambda p(x)\right)$ is again the solution of the Navier-Stokes equation. Plugging back in equations (A.1.5) and (A.2.19) we obtain

$$
\begin{aligned}
\left(u_{0} \cdot \nabla\right) u+\lambda(u \cdot \nabla) u & =-\nabla p+\mu \Delta u+f \\
\nabla \cdot u & =0 .
\end{aligned}
$$

Now applying the limit $\lambda \rightarrow 0$ we have the linearized form of the Navier-Stokes equation, i.e.,

$$
\begin{aligned}
\left(u_{0} \cdot \nabla\right) u & =-\nabla p+\mu \Delta u+f \\
\nabla \cdot u & =0 .
\end{aligned}
$$

These linearized equations are known as the Oseen equation named after Carl Wilhelm Oseen. It is observed the above pair of equations (A.2.22) and (A.2.23) reduces to a well known Stokes equation for $u_{0}=0$. Together with conditions (A.2.20) and (A.2.21), Stokes derived a remarkable and explicit solution $(u, p)$ in 1851 [44]. However this solution fails to demonstrate the wake region behind the obstacle. In 1910 Oseen found a paraboloidal wake region behind the obstacle, which is an important breakthrough in the field of fluid dynamics. 


\section{Bibliography}

[1] M. Abramowitz and I. A. Stegun, editors. Handbook of Mathematical Functions. Dover Publications, Inc., New York, 1964.

[2] R. A. Adams. Sobolev Spaces. Academic Press, 1975.

[3] S. N. Antontsev, A. V. Kazhikhov, and V. N. Monakhov. Boundary-Value Problems in Mechanics of Nonmhomogeneous Fluids. North-Holland Publishing Company, 1990.

[4] K. E. Atkinson. The numerical solution of integral equations of the second kind, volume 4 of Cambridge monographs on applied and computational mathematics. Cambridge University Press, 1997.

[5] G. K. Batchelor. An introduction To Fluid Dynamics. Cambridge Univ Press, 1967.

[6] M. F. Ben Hassen, K. Erhard, and R. Potthast. The point-source method for 3D reconstructions for the Helmholtz and Maxwell equations. Inverse Problems, 22(1):331-353, 2006.

[7] V. I. Burenkov. Sobolev Spaces on Domains. B. G. Teubner Stuttgart . Leipzig, 1998.

[8] T. J. Chung. Computational Fluid Dynamics. Cambridge University Press New York, 2010.

[9] H. W. Engl, M. Hanke, and A. Neubauer. Regularization of inverse problems, volume 375 of Mathematics and its Applications. Kluwer Academic Publishers Group, Dordrecht, 1996.

[10] K. Erhard. Point Source Approximation Methods in Inverse Obstacle Reconstruction Poblems. PhD thesis, University of Göttingen, 2005.

[11] K. Erhard and R. Potthast. Reconstructing an inclusion from boundary measurements in electrical impedance tomography and acoustic scattering. Inverse Problems, 19:1139-1157, 2003. 
[12] L. C. Evans, editor. Partial Differential Equations. 1998.

[13] R. Finn and D. R. Smith. On the linearized hydrodynamical equations in two dimensions. Arch. Rational Mech. Anal, 25:1-25, 1967.

[14] G. B. Folland. Introduction to partial differential equations. Princeton University Press, Princeton, NJ, second edition, 1995.

[15] R. W. Fox and A. T. McDonald. Introduction To Fluid Mechanics. John Wiley \& Sons. Inc., 1992.

[16] G. P. Galdi. An Introduction to the Mathematical Theory of the Navier-Stokes Equations. Springer, New York, 1994.

[17] W. Hackbusch. Integralgleichungen Theorie und Numerik. Teubner Studienbcher Mathematik., 1989.

[18] J. Hadamard. Lectures on cauchy's problem in linear partial differential equations. Yale University Press, New Haven, 1923.

[19] M. Ikehata. Reconstruction of an obstacle from the scattering amplitude at a fixed frequency. Inverse Problems, 14(4):949-954, 1998.

[20] M. Ikehata. Reconstruction of the shape of the inclusion by boundary measurements. Comm. PDE, 23:1459-1474, 1998.

[21] R. Kress. Numerical Analysis, volume 181 of Graduate Texts in Mathematics. Springer, 1998.

[22] R. Kress. Linear integral equations, volume 82 of Applied Mathematical Sciences. Springer-Verlag, New York, second edition, 1999.

[23] R. Kress and S. Meyer. An inverse boundary value problem for the oseen equation. Math. Meth. Appl. Sci., 23:103-120, 2000.

[24] L. Kühn. Magnetic Tomography - On the Nullspace of the Biot-Savart Operator and Point Sources for Field and Domain Reconstruction. phd thesis, University of Göttingen, 2005.

[25] J. Liu and R. Potthast. On the duality of the potential method and the point source method in inverse scattering problems. J. Integral Equations Appl., 21:297-315, 2009 .

[26] J. J. Liu, G. Nakamuray, and R. Potthast. A new approach and improved error analysis for reconstructing the scattered wave by the point source method. 2003. 
[27] D. R. Luke and R. Potthast. The no response test - a sampling method for inverse scattering problems. SIAM Journal on Applied Mathematics, 63(4):1292$1312,2003$.

[28] D. R. Luke and R. Potthast. The point source method for inverse scattering in the time domain. Math. Meth. Appl. Sci., 29, Issue 13:1501-1521, 2006.

[29] W. McLean. Strongly Elliptic Systems and Boundary Integral Equations. Cambridge University Press, 2000.

[30] S. Meyer. Analytische und Numerische Untersuchungen Eines Inversen Randwertproblems Für Die Oseen-Gleichung. PhD thesis, University of Göttingen, 1998.

[31] V. A. Morozov. On the solutions of functional equations by the method of regularization. Soviet Math. Doklady, 7:414-417, 1966.

[32] C. W. Oseen. Über die stokessche formel und Über eine verwandte aufgabe in der hydrodynamik. Ark. Mat. Astron. Fys., 6(29):1-20, 1910.

[33] C. W. Oseen. Neuere Methoden und Ergebnisse in der. Hydrodynamik. Akademische Verlagsgesellschaft m. b. H, 1927.

[34] R. Potthast. A fast new method to solve inverse scattering problems. Inverse Problems, 12(5):731-742, 1996.

[35] R. Potthast. A point-source method for inverse acoustic and electromagnetic obstacle scattering problems. IMA J. Appl. Maths., 61:119-140, 1998.

[36] R. Potthast. Point sources and multipoles in inverse scattering theory. Habilitation thesis, Göttingen, 1999.

[37] R. Potthast. A set-handling approach for the no-response test and related methods. Mathematics and Computers in Simulation, 66:281-295, 2004.

[38] R. Potthast. A survey on sampling and probe methods for inverse problems. Inverse Problems, 22(2):R1-R47, 2006.

[39] R. Potthast. On the convergence of the no response test. SIAM J. Math. Anal., 38 no. $6: 1808-1824,2007$.

[40] R. Potthast. An iterative contractive framework for probe methods (lasso). preprint, 2011.

[41] R. Potthast, J. Sylvester, and S. Kusiak. A 'range test' for determining scatterers with unknown physical properties. Inverse Problems, 19(3):533-547, 2003. 
[42] J. Schulz. Field reconstructions and range tests for acoustics and electromagnetics in homogeneous and layered media. PhD thesis, University of Göttingen, 2007.

[43] G. G. Stokes. On the theories of the internal friction of fluids in motion. Cambridge Phil. Soc., 8:287-319, 1845.

[44] G. G. Stokes. On the effect of the intenal fricition of fluids on the motion of pendulums. Trans. Cambridge Phil. Soc., 9:8-106, 1851.

[45] G. N. Watson. Theory of Bessel functions. Cambridge University Press, 1922. 


\title{
Curriculum Vitae/Lebenslauf
}

\section{Personal Data}

\author{
Name: $\quad$ Qazi Muhammad Zaigham Zia \\ Date of Birth: $\quad 21.10 .1982$ \\ Place of Birth: $\quad$ Rawalpindi (Bhoner Kaswal) \\ Nationality: Pakistan \\ Marital Status: Married \\ Parents: $\quad$ Qazi Muhammad Zia-Ur-Rahman \\ Yasmin Younis \\ Spouse Name: Mubeen Akhtar

\section{Primary and Secondary Education} \\ 1985-1994: $\quad$ Mumtaz Memorial Public School, Islam Pura Jabbar, \\ Gujar Khan, District Rawalpindi \\ 1994-1996: $\quad$ Govt. High School, Bewal, \\ Gujar Khan, District Rawalpindi \\ 1996-1998: $\quad$ Govt. Gordon College, Rawalpindi
}

\section{Higher Education}

1998-2000: $\quad$ B.Sc. in Mathematics and Statistics

from University of the Punjab, Lahore

2001-2002: $\quad$ M.Sc. in Mathematics

from Quaid-i-Azam University Islamabad

2003-2005: $\quad$ M.Phil in Mathematics

from Quaid-i-Azam University Islamabad

\section{Occupational Career}

2005-2006: $\quad$ Lecturer, Department of Mathematics,

COMSATS Institute of Information Technology, Islamabad 